\title{
Nicotinamide mononucleotide (NMN) deamidation by the gut microbiome and evidence for indirect upregulation of the $\mathrm{NAD}^{+}$metabolome
}

Lynn-Jee Kim ${ }^{1}$, Timothy J. Chalmers ${ }^{1}$, Greg C. Smith ${ }^{1}$, Abhirup Das ${ }^{1}$, Eric Wing Keung Poon ${ }^{2}$, Jun Wang ${ }^{2,3}$, Simon P. Tucker $^{4}$, David A. Sinclair ${ }^{1,5}$, Lake-Ee Quek $^{6^{*}}$, Lindsay E. $\mathrm{Wu}^{1 *}$

${ }^{1}$ School of Medical Sciences, UNSW Sydney, NSW 2052, Australia

${ }^{2}$ GeneHarbor (Hong Kong) Biotechnologies Limited, Hong Kong Science Park, Shatin, Hong Kong SAR, China

${ }^{3}$ School of Life Sciences, The Chinese University of Hong Kong, Hong Kong SAR, China

${ }^{4}$ Jumpstart Fertility Pty Ltd, Melbourne, Victoria, Australia

${ }^{5}$ Harvard Medical School, Boston, MA, United States of America

${ }^{6}$ School of Mathematics and Statistics, The University of Sydney, NSW 2007, Australia

*These authors contributed equally to this work. Correspondence: lindsay.wu@unsw.edu.au +61 293851621

\section{ABSTRACT}

Treatment with nicotinamide mononucleotide (NMN) is a prominent strategy to address the agerelated decline in nicotinamide adenine dinucleotide $\left(\mathrm{NAD}^{+}\right)$levels for maintaining aspects of latelife health. It is assumed that exogenous NMN is directly incorporated into $\mathrm{NAD}^{+}$in mammals by the canonical recycling pathway, however the need for $\mathrm{NAD}^{+}$is conserved across evolution, including bacteria in the gut microbiome, which can deamidate NMN to nicotinic acid mononucleotide $(\mathrm{NaMN})$. Here, we use strategic isotope labelling studies to demonstrate a role for the gut microbiome in deamidating orally delivered NMN into NaMN prior to its uptake and incorporation in mammals. Microbiome depletion increased the overall abundance of NAD metabolites, suggesting a competition relationship. Strikingly, treatment with labelled NMN increased the production of unlabelled NAD precursors, with a greater than 3-fold increase in endogenous NR levels in the gut of antibiotics treated animals upon labelled NMN treatment. These data suggest that exogenous NMN impacts the NAD metabolome through indirect means, rather than through its direct incorporation, including through the production of endogenous NR via an as-yet unidentified pathway, and demonstrate an important role for the gut microbiome in the assimilation of orally delivered NMN. 


\section{INTRODUCTION}

Nicotinamide adenine dinucleotide $\left(\mathrm{NAD}^{+}\right)$is an essential redox cofactor central to metabolic processes such as glycolysis, the tricarboxylic (TCA) cycle and fatty acid oxidation (Xiao et al., 2018; Ying, 2008). $\mathrm{NAD}^{+}$is also consumed by enzymes such as the sirtuins (Imai et al., 2000) and poly(ADP-ribose) polymerase (PARP) enzymes (Kim et al., 2005) which are mediators of genome stability (Haigis and Sinclair, 2010) and DNA repair (de Murcia and de Murcia, 1994). Given the essential role of this metabolite, the decline in $\mathrm{NAD}^{+}$that occurs during biological ageing (Bertoldo et al., 2020; Braidy et al., 2011; Camacho-Pereira et al., 2016; Clement et al., 2019; Gomes et al., 2013; Massudi et al., 2012) and disease states (Frederick et al., 2016; Jukarainen et al., 2016; Ryu et al., 2016) has gained attention as a target for therapeutic intervention (Rajman et al., 2018). Strategies to boost $\mathrm{NAD}^{+}$levels through supplementation with NAD precursors such as nicotinamide mononucleotide $(\mathrm{NMN})$ and nicotinamide riboside (NR) are emerging as promising therapeutics (Bertoldo et al., 2020; Cantó et al., 2012; Das et al., 2018; Mills et al., 2016; Okabe et al., 2019; Rajman et al., 2018; Tarantini et al., 2019; Yoshino et al., 2018; Zhang et al., 2016). Historically, dietary supplementation with the NAD precursors nicotinic acid $(\mathrm{Na})$ or nicotinamide $(\mathrm{Nam})$ has been used to prevent chronic NAD deficiency, which causes pellagra. When these micronutrients are replete, the step converting Nam into NMN by the enzyme nicotinamide phosphoribosyltransferase (NAMPT) is rate limiting in NAD synthesis (Revollo et al., 2004), and the use of NAD precursors that occur after this step, namely NMN and NR, have gained prominence as a strategy to raise $\mathrm{NAD}^{+}$.

Oral delivery is the primary route for administration of NMN and NR in rodents (Das et al., 2018; Mills et al., 2016; Trammell et al., 2016; Yoshino et al., 2011) and humans (Martens et al., 2018; Trammell et al., 2016). It is important to understand how orally administered NMN is metabolised. Intravenously_ but not orally_administered NR and NMN was shown to be delivered to tissues intact, due to rapid first pass metabolism by the liver, which hydrolysed NMN and NR into free Nam (Liu et al., 2018). Given that the premise of providing NR or NMN rather than Nam is that these 
metabolites overcome the rate-limiting step of NAMPT (Rajman et al., 2018), it is unclear why oral delivery of these compounds - which are rapidly metabolised to provide free Nam - raises $\mathrm{NAD}^{+}$ levels more than treatment with Nam, which is abundant in dietary sources.

Given the highly conserved requirement for $\mathrm{NAD}^{+}$across evolution (Gazzaniga et al., 2009), it could be expected that microbial species in the gut microbiome would consume or metabolise NAD precursors obtained from dietary sources or orally delivered bolus doses. This was recently described for Nam, which can undergo deamidation to nicotinic acid $\mathrm{Na}$ ) in the gut by the bacterial enzyme PncA (Shats et al., 2020). We sought to explore whether the microbiome would play a similar role in the metabolism of orally delivered NMN, by tracing the metabolism of isotope labelled NMN in the intestinal tissue of mice during the context of microbiome ablation. If so, this could help explain the striking appearance of nicotinic acid adenine dinucleotide (NaAD) upon in vivo supplementation with NR (Trammell et al., 2016). In mammals, NR is phosphorylated into NMN by NR kinases (NRK1/2) (Bieganowski and Brenner, 2004; Ratajczak et al., 2016a), and then adenylated into NAD ${ }^{+}$by NMNAT enzymes (NMNAT1-3) (Berger et al., 2005; Emanuelli et al., 2001; Garavaglia et al., 2002; Raffaelli et al., 2002; Schweiger et al., 2001; Zhang et al., 2003), effectively bypassing NaAD, which is an intermediate of the Preiss-Handler pathway (i.e., de novo synthesis) (Preiss and Handler, 1958a; Preiss and Handler, 1958b). In contrast, bacteria have a well-characterised NMN deamidase enzyme, PncC (Galeazzi et al., 2011). A simple theory to explain the increased NaAD with NR treatment (Trammell et al., 2016) could be that NMN and NR assimilation follows a non-canonical route that combines steps of both microbial and mammalian processes, whereby NMN is de-amidated into $\mathrm{NaMN}$ or NaR prior to its uptake into mammalian tissue, where it is assimilated into $\mathrm{NAD}^{+}$via the intermediate step of $\mathrm{NaAD}$. This theory could explain the appearance of NaAD following NR supplementation (Trammell et al., 2016). 
To determine whether the gut microbiome has a role in NMN metabolism, we designed a stable isotopologue of NMN, which was delivered to animals treated with antibiotics to ablate the gut microbiome. Targeted metabolomics was then used to measure the incorporation of labelled NMN into the NAD metabolome. Here, we present evidence for the deamidation of NMN by the gut microbiome. We further show that ablation of the microbiome by antibiotic treatment increases the uptake and conversion of orally delivered NMN into the NAD metabolome, and that isotope labelled NMN presents in the intestinal tissue overwhelmingly in the form of NR, suggesting that the dephosphorylation of NMN is the primary route for its uptake. Contrary to the assumption that exogenous NMN treatment raises $\mathrm{NAD}^{+}$levels through its direct incorporation into the NAD metabolome, we show that treatment with isotope labelled NMN results in the upregulation of endogenous, unlabelled NAD metabolites. Overall, our results demonstrate that the gut microbiome plays a role in the assimilation of NMN across the gastrointestinal tissue.

\section{RESULTS}

\section{NMN treatment alters the de novo arm of $\mathrm{NAD}^{+}$synthesis}

Previous work described that administration of the amidated NAD precursor NR resulted in the formation of the deamidated metabolites nicotinic acid mononucleotide $(\mathrm{NaMN})$ and nicotinic acid adenine dinucleotide (NaAD) (Trammell et al., 2016), deamidated intermediates in the de novo arm of NAD homeostasis. According to canonical models of mammalian NAD homeostasis, the metabolism of NR into $\mathrm{NAD}^{+}$does not intersect with the de novo pathway, however bacteria present in the gut microbiome do encode de-amidase enzymes such as PncC, which deamidates NMN into nicotinic acid mononucleotide (NaMN) for metabolism via the de novo pathway (Galeazzi et al., 2011). One possibility is that NR treatment results in the previously described spike in NaMN and $\mathrm{NaAD}$ as a result of deamidation by the gut microbiome, followed by phosphorylation into NaMN and adenylation into NaAD by the mammalian enzymes NRK1/2 and NMNAT1-3. Given that NMN is taken up following its extracellular dephosphorylation into NR (Ratajczak et al., 2016a), a similar 
trend might be expected with NMN treatment. To test this idea in vivo, we used mice that were exposed to a course of antibiotics to ablate the gut microbiome (Supp. Fig. 1). These animals received a bolus of NMN $(500 \mathrm{mg} / \mathrm{kg})$ by oral gavage, and four hours later, animals were sacrificed and tissues rapidly preserved for targeted metabolomic analysis (Fig. 1). In agreement with previous work, NMN treatment increased the abundance of the de-amidated metabolites $\mathrm{NaR}$ and NaMN in both the GIT (Fig. 1a, b) and liver (Fig. 1d, e), while NaAD was increased in the liver (Fig. 1f) as consistent with previous work (Trammell et al., 2016). Interestingly, this was completely abolished in antibiotic treated animals, where NMN treatment instead led to a spike in the amidated metabolites NR (Fig. 1g, j) and NMN (Fig. 1h, k), and abolished the increase in liver NaAD (Fig. 1f). To highlight the inverse relationship between amidated and de-amidated metabolites during antibiotics treatment, the abundance of each deamidated metabolite was expressed as a ratio of its amidated counterpart (Fig. 1m-r), highlighting a profound role for the microbiome in dictating the roles of the de-amidated and amidated arms of NAD metabolism.

\section{Strategic isotope tracing of NMN metabolism}

While the standard assumption of studies that deliver a bolus of exogenous metabolites such as NR or NMN is that changes in downstream metabolites are due to the direct incorporation of exogenous material, we next sought to carefully test whether this exogenous NMN was indeed undergoing direct deamidation prior to its incorporation into the NAD metabolome using isotope tracing studies. We designed two separate stable isotopologues of NMN that were strategically labelled at positions that would answer our question of whether NMN was incorporated following its deamidation. The first of these, designated as NMN1, was ${ }^{13} \mathrm{C}$ labelled at all five carbon positions of the ribose moiety for an $\mathrm{M}+5$ mass shift, and ${ }^{15} \mathrm{~N}$ labelled at the nicotinyl ring for an overall $\mathrm{M}+6$ mass shift (Fig. 2a, Supp. Fig. 2). In the last step of the de novo pathway, the enzyme NAD synthetase (NADS) amidates the carboxylic acid of $\mathrm{NaAD}$ using an ammonia intermediate derived from the amide group of glutamine, yielding glutamate (Fig. 2). By delivering the NMN1 (M+6) isotope in the presence of ${ }^{15} \mathrm{~N}$-glutamine 
$(\mathrm{M}+1)$ labelled at the amide position, the presence of $\mathrm{M}+7$ labelled $\mathrm{NAD}^{+}$with an additional mass shift from the nicotinyl amide would indicate that the original amide $\mathrm{N}$ atom had been lost during deamidation and replaced by a ${ }^{15} \mathrm{~N}$ atom from ${ }^{15} \mathrm{~N}-\mathrm{Gln}$, indicating incorporation of NMN into $\mathrm{NAD}^{+}$ via prior deamidation and the de novo pathway (Fig. 2a). To complement this experiment, we designed a second isotope, designated as NMN2, where all five carbons of the ribose moiety were ${ }^{13} \mathrm{C}$ labelled, and both the nicotinyl ring and amide positions were ${ }^{15} \mathrm{~N}$ labelled, for an overall $\mathrm{M}+7$ mass shift (Fig. 2b, Supp. Fig. 2). When delivered in a separate experiment, if NMN2 (M+7) underwent deamidation prior to its incorporation, the ${ }^{15} \mathrm{~N}$ amide would be lost and replaced by a ${ }^{14} \mathrm{~N}$ atom from the endogenous glutamine pool, resulting in $\mathrm{M}+6$ labelled $\mathrm{NAD}^{+}$. By comparing the ratios of $\mathrm{M}+7$ and $\mathrm{M}+6$ labelled $\mathrm{NAD}^{+}$in each experiment, we could quantify the proportion of NMN that had been incorporated into $\mathrm{NAD}^{+}$following deamidation and assimilation by the de novo pathway. This would be supported by comparing the ratios of $\mathrm{M}+1$ and $\mathrm{M}+2$ labelled Nam, which is released by $\mathrm{NAD}^{+}$consuming enzymes, however this interpretation would be complicated by the recently described role of the bacterial nicotinamide (Nam) deamidase PncA in systemic mammalian $\mathrm{NAD}^{+}$ homeostasis (Shats et al., 2020). By using triple-quad mass spectrometry and multiple reaction monitoring (MRM) for targeted metabolomics, we could further refine these data to determine where mass shifts occurred, including whether Nam was labelled at the base (i.e. nicotinyl ring) or amide positions, and whether $\mathrm{M}+6$ or $\mathrm{M}+7$ labelling of NAD was from the NMN rather than the AMP moiety.

\section{${ }^{15} \mathrm{~N}-$ Gln labelling of NAD ${ }^{+}$biosynthesis}

To test whether this scheme would lead to labelling of the NAD pool as anticipated, we first used primary rat hepatocytes grown in vitro, to avoid contributions from the microbiome. Hepatocytes were treated for $24 \mathrm{hr}$ with ${ }^{15} \mathrm{~N}$-glutamine $(\mathrm{M}+1)$ in the presence or absence of NMN1 $(\mathrm{M}+6)$, or with NMN2 (M+7) (Fig. 3). Cell lysates were subject to targeted metabolomic analysis to assess the degree of isotope incorporation into each metabolite (Fig. 3a-e). Delivery of each of these isotopes yielded 
the expected $\mathrm{M}+6$ and $\mathrm{M}+7$ mass shifts of NMN (Fig. 3b) as well as its de-phosphorylated counterpart NR (Fig. 3a), which is consistent with the indirect transport of NMN (Ratajczak et al., 2016a) (Bieganowski and Brenner, 2004; Ratajczak et al., 2016), though these data do not exclude the direct transport of NMN via the putative transporter SLC12A8 (Grozio et al., 2019) - for this reason, the data in this investigation could be interpreted as evidence for deamidation of NMN and/or NR, rather than NMN alone. To test the strategy of using ${ }^{15} \mathrm{~N}$ glutamine to label $\mathrm{NAD}^{+}$synthesis, we compared the ratio of $\mathrm{M}+1$ (nicotinamide labelled) to $\mathrm{M}+0$ (endogenous) $\mathrm{NAD}^{+}$(Fig. 3f). As expected, ${ }^{15} \mathrm{~N}-\mathrm{Gln}$ treatment increased $\mathrm{M}+1$ labelling of endogenous $\mathrm{NAD}^{+}$, with $\mathrm{M}+1 \mathrm{NAD}^{+}$labelling in untreated samples due to baseline levels of naturally occurring isotopes. High levels of $\mathrm{M}+1 \mathrm{NAD}^{+}$labelling (Fig. 3f) were observed in samples treated with NMN1, likely due to recycling of the M+1 labelled Nam moiety (Fig. 3g) following the breakdown of $\mathrm{NAD}^{+}$(Fig. 2), or by cleavage of the NMN glycosidic bond between the ribose and nicotinamide groups. As expected, treatment with NMN1 $(\mathrm{M}+6)$ and NMN2 $(\mathrm{M}+7)$ led to $\mathrm{M}+6$ and $\mathrm{M}+7$ labelling of $\mathrm{NAD}^{+}$(Fig. 3c). While we had hypothesised that in vivo treatment with $\mathbf{N M N 1}(\mathrm{M}+6)$ and ${ }^{15} \mathrm{~N}-\mathrm{Gln}(\mathrm{M}+1)$ would lead to $\mathrm{M}+7$ labelled $\mathrm{NAD}^{+}$due to the deamidation of $\mathrm{NMN}$ by the gut microbiome, in this culture of primary hepatocytes alone we observed that ${ }^{15} \mathrm{~N}-\mathrm{Gln}$ co-treatment with NMN1 $(\mathrm{M}+6)$ increased the formation of M+7 labelled NAD ${ }^{+}$, when compared to NMN1 $(\mathrm{M}+6)$ alone (Fig. 3h). In line with the expected recycling of labelled Nam from $\mathrm{NAD}^{+}$(Fig. 2a), this increased formation of $\mathrm{M}+7$ labelled $\mathrm{NAD}^{+}$ during NMN1 $(\mathrm{M}+6)$ and ${ }^{15} \mathrm{~N}-\mathrm{Gln}$ co-treatment was matched by an identical increase in $\mathrm{M}+2$ labelling of free Nam (Fig. 3i), which was incorporated into the nicotinyl moiety of $\mathrm{NAD}^{+}$(Fig. 3j). ${ }^{15} \mathrm{~N}-\mathrm{Gln}$ treatment increased $\mathrm{M}+1$ labelling at the amide position of Nam (Fig. 3i), but not the base (i.e. nicotinyl ring) position (Fig. 3k), which does not undergo substitution by NADS, with NMN1 (Fig. 2a) treatment serving as a positive control for labelling at this position. Overall, these data verified our system of labelling, and demonstrated the specificity of our targeted analytical approach, based on triple quadrupole mass spectrometry and MRM targeted metabolomics. While the production of $\mathrm{M}+7$ labelled $\mathrm{NAD}^{+}$during treatment with $\mathbf{N M N 1}(\mathrm{M}+6)$ and ${ }^{15} \mathrm{~N}-\mathrm{Gln}(\mathrm{M}+1)$ point to 
the deamidation of either NMN or NR by mammalian cells in vitro, these data suggest that as a proportion of the overall cellular pool, the canonical recycling pathway dominates as the primary pathway for incorporation of exogenous NMN. As mammals do not encode a known NMN, NR or Nam deamidase enzyme, we next sought to measure the incorporation of labelled NMN into the $\mathrm{NAD}^{+}$metabolome of bacteria, which encode the NMN deamidase PncC (Galeazzi et al., 2011) and the Nam deamidase PncA (Frothingham et al., 1996).

\section{NMN deamidation by bacteria}

Unlike mammals, bacteria rely on an $\mathrm{NAD}^{+}$dependent DNA ligase that is inhibited by NMN, the product of its own reaction (Chen et al., 2002; Geider, 1972; Zimmerman and Oshinsky, 1969), resulting in the accumulation of intracellular NMN during exponential growth (Olivera and Lehman, 1967). NMN is salvaged through the bacterial NMN deamidase PncC, yielding NaMN as a substrate for $\mathrm{NAD}^{+}$synthesis by the Preiss-Handler pathway (Galeazzi et al., 2011). To model whether orally delivered NMN would undergo deamidation by bacteria, growth phase E. coli cultures were supplemented with NMN1 (M+6) (Fig. 2a) and subjected to targeted metabolomics of both cell lysates and extracellular culture media (Fig. 4). Consistent with the role of PncC in NMN metabolism in bacteria, treatment with labelled NMN resulted in the rapid incorporation of isotope labels into NaMN, with over 50 times the quantity of labelled NaMN compared to NMN (Fig. 4). A role for the Nam de-amidase PncA is strikingly reflected in the abundance of nicotinic acid (Na) compared to nicotinamide (Nam) in the cell pellet compared to the culture supernatant, where the abundance of Nam and absence of $\mathrm{Na}$ in growth media was completely reversed in cell pellets, indicating rapid Nam deamidation. Overall, the rapid and abundant labelling of the deamidated metabolite NaMN by E. coli (Fig. 4) supported our hypothesis that the gut microbiome could contribute to the metabolism of orally administered NAD precursors such as NMN.

\section{Antibiotic treatment alters NMN deamidation in vivo}


To directly trace whether the increase in de-amidated metabolites following NMN administration (Fig. 1) was indeed due to the direct deamidation and incorporation of these metabolites, we next delivered our strategically designed isotopes into animals that had similarly been treated with antibiotics to deplete the gut microbiome, as confirmed by reductions in faecal DNA concentration (Supp. Fig. 1a), full-length 16S rRNA sequencing (Supp. Fig. 1c) and reduced alpha diversity (Supp. Fig. 1e-r). Following antibiotic treatment, animals received a single oral gavage $(50 \mathrm{mg} / \mathrm{kg})$ of the NMN1 (M+6) isotope (Fig. 2a), in parallel with an i.p. bolus of ${ }^{15} \mathrm{~N}-\mathrm{Gln}(\mathrm{M}+1)$. Four hours later, animals were sacrificed and tissues rapidly preserved for targeted metabolomic analysis (Fig. 5, Supp. Fig. 3-5) to measure the formation of $\mathrm{M}+7$ labelled $\mathrm{NAD}^{+}$, which would be indicative of prior deamidation and incorporation via the de novo pathway as described in Fig 1 and Fig. 2. To complement this experiment, a separate cohort of antibiotic treated animals (Supp. Fig. 1b, d) received a bolus of the NMN2 (M+7) isotope (Fig. 2b), following which tissues were similarly collected $4 \mathrm{hr}$ later for targeted metabolomic analysis (Fig. 6, Supp. Fig. 6, 7) to measure the loss of the NMN2 amide label from $\mathrm{M}+7$ to $\mathrm{M}+6$ labelled $\mathrm{NAD}^{+}$, which would also indicate deamidation prior to incorporation.

In tissues from animals treated with NMN1 $(\mathrm{M}+6)$, the deamidation of NMN could be quantified by comparing the ratio of $\mathrm{NAD}^{+}$that incorporated the expected $\mathrm{M}+6$ labelling, to the proportion of $\mathrm{NAD}^{+}$that incorporated an extra mass shift from co-treatment with ${ }^{15} \mathrm{~N}-\mathrm{Gln}(\mathrm{M}+1)$, resulting in $\mathrm{M}+7$ $\mathrm{NAD}^{+}$(Fig. 2a, 7a), with an increased ratio of $\mathrm{M}+7$ to $\mathrm{M}+6$ labelled $\mathrm{NAD}^{+}$indicating deamidation of NMN. The reason for using these ratios, rather than the overall amounts of each isotope (Fig. 5, Supp. Fig. 4,5 ), is that these ratios act as internal controls for differences in bioavailability following antibiotic treatment, as discussed in later sections. From the intact labelling of $\mathrm{NAD}^{+}$in the GIT from NMN1 treatment, around 13\% was M+7 labelled (Fig. 7b, c). Importantly, these data likely underestimates incorporation via the de-amidated route, as this scheme relied on the availability of exogenous ${ }^{15} \mathrm{~N}-\mathrm{Gln}$ relative to the endogenous pool of unlabelled Gln, with labelled Gln composing 
only $9-13 \%$ of the total plasma Gln pool at the experimental endpoint (Supp. Fig. 3g). Consistent with our hypothesis, the ratio of $\mathrm{M}+7$ to $\mathrm{M}+6$ labelling in the GIT was reduced in antibiotic treated animals (Fig. 7b), suggesting reduced deamidation of orally administered NMN when contributions from the microbiome were reduced. This was reflected by a reduction in the ratio of $\mathrm{M}+2$ to $\mathrm{M}+1_{\text {base }}$ labelled Nam in both the GIT, liver and plasma (Fig. 7d, e, Supp. Fig. 8h), however this change also likely reflected reduced contributions from the bacterial nicotinamide de-amidase PncA (Frothingham et al., 1996) following antibiotic treatment (Shats et al., 2020). This change in Nam labelling carried into the $\mathrm{M}+2$ or $\mathrm{M}+1_{\text {base }}$ labelling of $\mathrm{NMN}$ (Fig. 7f, g) and $\mathrm{M}+2$ labelling of $\mathrm{NAD}^{+}$ (Fig. 7h, i), with reduced labelling ratios of these recycled isotopes during antibiotic treatment likely reflecting a combination of possible NMN/NR deamidation (Fig. 7a, b), and contributions from the bacterial Nam deamidase PncA (Shats et al., 2020).

To complement this approach, in the NMN2 (M+7) experiment (Fig. 2b), we would anticipate that deamidation by the microbiome would result in loss of the ${ }^{15} \mathrm{~N}$ amide label, resulting in the formation of $\mathrm{M}+6 \mathrm{NAD}^{+}$at the expense of $\mathrm{M}+7 \mathrm{NAD}^{+}$(Fig. $2 \mathrm{~b}, 7 \mathrm{j}$ ). In contrast to the previous NMN1 experiment, the ratios of $\mathrm{M}+7$ to $\mathrm{M}+6 \mathrm{NAD}^{+}$would instead decrease as the rate of deamidation increased. Further, interpretation of deamidation in this NMN2 experiment was not limited by the availability of exogenous ${ }^{15} \mathrm{~N}-\mathrm{Gln}$ relative to a large, endogenous pool of Gln, as was the case with the NMN1 experiment (Fig. 7a, Supp. Fig. 3). In this experiment, the ratio of $M+7$ to $M+6 \mathrm{NAD}^{+}$ was around 3:1 (Fig. 7k, 1), suggesting that around $25 \%$ of orally administered NMN undergoes deamidation prior to its intact incorporation into $\mathrm{NAD}^{+}$. In support of a role for the microbiome in $\mathrm{NMN}$ deamidation, the ratio of $\mathrm{M}+7$ to $\mathrm{M}+6$ labelled $\mathrm{NAD}^{+}$was increased in the GIT and liver of antibiotic treated animals (Fig. $7 \mathrm{k}, 1)$. This was similarly matched by an increased ratio of $\mathrm{M}+2$ to

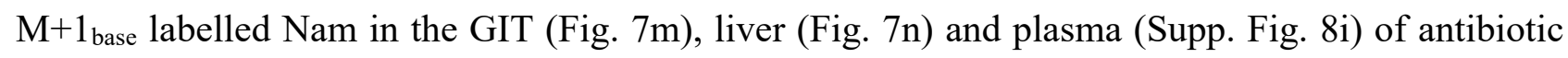
treated animals, reflecting decreased incorporation following deamidation, though this could instead be due the deamidation of Nam rather than NMN. As in the previous experiment, these labels were 
recycled into $\mathrm{M}+2$ labelled $\mathrm{NMN}\left(\right.$ Fig. 7o, p) and $\mathrm{NAD}^{+}$(Fig. 7q, r). In addition to differences in the isotope labelling of $\mathrm{NAD}^{+}$(Fig. 7), these experiments replicated the inverse relationship between NaMN and NMN levels following antibiotic treatment (Fig. 5a, e, Fig. 7a, e) observed in our earlier experiment with unlabelled NMN (Fig. 1b, h, n). Overall, these data from two complementary approaches support the concept that orally delivered NMN or NR can undergo deamidation prior to incorporation, and a role for the microbiome in mediating this. While these data could in part explain the spike in the de-amidated metabolites $\mathrm{NaMN}$ and $\mathrm{NaAD}$ following treatment with the amidated precursors NR (Trammell et al., 2016) or NMN (Fig. 1), it is important to note that when measured as a proportion of the overall $\mathrm{NAD}^{+}$pool, the contribution of both $\mathrm{M}+7$ and $\mathrm{M}+6$ intact labelled $\mathrm{NAD}^{+}$was small. Partially labelled $\mathrm{NAD}^{+}(\mathrm{M}+2)$ was around 10 -fold more abundant than intact labelled NAD ${ }^{+}(\mathrm{M}+7)$ (Fig. 5c, 6c, Supp. Fig. 4-7), indicating either cleavage of the labile glycosidic bond of $\mathrm{NMN}$, or rapid recycling of $\mathrm{NAD}^{+}$, as described previously (Liu et al., 2018). Following cleavage of the glycosidic bond to release free Nam, its deamidation in the GIT (Shimoyama et al., 1971; Tanigawa et al., 1970) by the bacterial enzyme PncA (Shats et al., 2020) also likely contributes to these changes.

\section{Exogenous NMN boosts the endogenous NAD ${ }^{+}$metabolome}

The abundance of partially labelled $\mathrm{NAD}^{+}$at the Nam position only is consistent with previous findings (Liu et al., 2018) that orally delivered NMN and NR undergo cleavage at the glycosidic bond to release free Nam, with only a small proportion of orally delivered material being incorporated into tissues intact. Given that NMN and NR are overwhelmingly incorporated in the form of free Nam, why do the downstream biological effects of NR or NMN differ from the delivery of free Nam (Trammell et al., 2016), a widely available nutrient present in dietary sources? One basic assumption is that exogenous $\mathrm{NAD}^{+}$precursors increase levels of NAD and other metabolites due to their direct incorporation, as expected by classic mass-balance models. Our data complicates this assumption, as delivery with exogenous NMN that was $100 \%$ isotope labelled (M+6 or M+7, Supp. Fig. 2) also 
increased the levels of unlabelled $(\mathrm{M}+0)$, endogenous metabolites. For example, treatment with NMN1 or NMN2 in antibiotics treated animals increased levels of unlabelled NaR (Fig. 5f, 6f, Supp. Fig. 4f, 6f) in the GIT, and increased unlabelled NR (Fig. 5b, 6b Supp. Fig. 4b, 6b) and NaAD (Fig. 5n, 6n, Supp. Fig. 5g, 7g) in the liver. Notably, treatment with labelled NMN in antibiotic treated animals increased the production of unlabelled NR in the GIT by over an order of magnitude (Fig. 5b, 6b, Supp. Fig. 4b, 6b).

\section{Host-microbe interactions in the bioavailability of orally delivered $\mathrm{NAD}^{+}$precursors}

Another unexpected aspect of these data was the overall increase in levels of these metabolites as a result of antibiotic treatment alone, which more than doubled the labelling of the metabolites NMN, NR, NAD ${ }^{+}$and Nam (Fig. 1g-i, 5a-d, 6a-d, Supp. Fig. 4h-k, 5h-k, 6h-k, 7h-k). This increase even occurred in the levels of unlabelled metabolites in animals that did not receive exogenous NMN (Supp. Fig. 4a-d, 6a-d). When the NMN1 $(\mathrm{M}+6)$ isotope was delivered, the incorporation of exogenous labels into $\mathrm{NAD}^{+}$metabolites was vastly increased in antibiotic treated animals, by an order of magnitude for some metabolites such as NR (Fig. 5b, Supp. Fig. 4i), a trend that was precisely recapitulated in a separate cohort of animals receiving the NMN2 (M+7) isotope (Fig. 6b, Supp. Fig. 6i) and in animals that received unlabelled NMN (Fig. 1g). The overwhelming abundance of NR in the GIT during antibiotics treatment is worthy of later investigation, as the abundance of this single metabolite was greater than all other $\mathrm{NAD}^{+}$metabolites combined, including NAD ${ }^{+}$itself.

\section{NMN uptake is facilitated by dephosphorylation into NR}

In addition to a role for the microbiome in NMN uptake and deamidation, the design of this study allowed us to shed light around how NMN is transported into cells. This has been described to occur following dephosphorylation of NMN into NR by the cell surface enzyme CD73, prior to uptake by ENT nucleoside transporters and re-phosphorylation into NMN inside the cell by NRK1/2 (Supp. 
Fig. 9) (Bieganowski and Brenner, 2004; Ratajczak et al., 2016a). Alternatively, the solute carrier protein SLC12A8 has been described as a dedicated NMN transporter (Grozio et al., 2019). As with CD73 and ENT, SLC12A8is located on the apical side of the intestinal tissue. As both mechanisms could co-exist, the question is the degree to which each mechanism contributes to the uptake of NMN.

If the direct route via SLC12A8 prevailed, we would expect to see high levels of labelled (M+7 or $\mathrm{M}+6) \mathrm{NMN}$, with lesser uptake of labelled NR. In contrast, if the indirect transport of NMN following its dephosphorylation into NR was dominant, there would be a higher levels of NR labelling. In primary hepatocytes (Fig. 3), NMN1 $(\mathrm{M}+6)$ treatment resulted in near complete labelling of the NR pool (Fig. 2b), with slightly lesser labelling of the NMN pool (Fig. 2a). Strikingly, in vivo treatment showed strong labelling of the NR, but not NMN pools (Fig. 5 a, b; Fig. 6 a, b; Supp. Fig. 4a-b, h-i; Supp. Fig. 6a-b, h-i; Supp. Fig. 9). Intact (M+6 or M+7) labelled NR levels (Supp. Fig. 4i, 6i) were five-fold higher than endogenous NR (M+0) (Fig. 5b, 6b, Supp. Fig. 4h, 5h; Supp. Fig. 9), which increased to a ten-fold greater enrichment with antibiotics treatment (Supp. Fig. 9). In stark contrast, only around 5\% of the NMN pool was M+7 labelled (Fig. 5a, Fig. 6a; Supp. Fig. 4a, h; Supp. Fig. 7a, h, Supp. Fig. 8). The inability of exogenous NMN to displace the endogenous NMN pool, combined with the surge of labelled NR, suggests that NMN uptake bypasses direct transport, and would instead support the dephosphorylation of NMN into NR to facilitate its intestinal absorption (Supp. Fig. 9). If direct transport of NMN does occur, it is in competition with the microbiome, as even when $\mathrm{M}+6$ or M+7 labelling of NMN was observed at low levels, this only occurred in antibiotic treated animals (Fig. 5b, 6b).

\section{Chronic NMN treatment does not alter microbial diversity}

Given the potential role for host-microbiome interactions in the metabolism of orally delivered NMN, it might be expected that NMN treatment would alter the composition of the microbiome. While $16 \mathrm{~S}$ rRNA sequencing (Supp. Fig. 2) of young animals that received an acute dose of exogenous NMN 
did not show a change in microbial diversity, this might not be expected in this timeframe and in young animals where $\mathrm{NAD}^{+}$is already replete. To test whether chronic NMN treatment could impact the composition of the gut microbiome, we treated aged, 97-week old animals with chronic NMN dosing via addition to drinking water ( $400 \mathrm{mg} / \mathrm{kg} /$ day) for $8-10$ weeks prior to cull (CamachoPereira et al., 2016; Clement et al., 2019; Massudi et al., 2012; Schultz and Sinclair, 2016). 16S rRNA long-read sequencing revealed no overall change in diversity of the gut microbiome (Fig. 8), however there were a number of changes among individual species, available in Supplementary File 1.

\section{DISCUSSION}

These studies were initially undertaken to address questions of how delivery of the amidated NAD precursors NMN or NR could lead to the surprising observation of a spike in the formation of a deamidated metabolite, NaAD (Trammell et al., 2016). In the canonical model of mammalian NAD biosynthesis, the amidated metabolites NMN, NR and Nam do not undergo deamidation into their counterparts nicotinic acid riboside (NaR), nicotinic acid mononucleotide (NaMN) and $\mathrm{Na}$ (Fig. 2). These deamidated forms are confined to the de novo and Preiss-Handler pathways, the final step of which involves the amidation of $\mathrm{NaAD}$ into $\mathrm{NAD}^{+}$by the enzyme NAD synthase (Fig. 2). The observation of increased NaAD following NR treatment (Trammell et al., 2016) could potentially be explained by feedback inhibition of further NAD biosynthesis to maintain NAD levels within a tight homeostatic threshold (Hara et al., 2019), resulting in a bottleneck at the enzyme NAD synthase (NADS) and accumulation of its substrate NaAD. Alternatively, bacteria possess deamidase enzymes not present in mammals, such as the NMN deamidase PncC (Galeazzi et al., 2011), which could alternatively contribute to the formation of $\mathrm{NaMN}$ and subsequently $\mathrm{NaAD}$. Our NMN tracers were specifically designed to answer these questions, with two independent, complementary approaches (Fig. 2) that would reflect incorporation into NAD ${ }^{+}$following its prior deamidation. In contrast to the "bottleneck" idea of NADS inhibition, the formation of M+7 labelled NAD ${ }^{+}$following NMN1 (M+6) treatment (Fig. 3h) suggest that NMN can be incorporated via the action of NADS. While some work 
from over half a century ago suggested the possible existence of a Nam de-amidase in mammalian liver (Petrack et al., 1965) that could account for this result from hepatocytes grown in vitro (Fig. 3), to date there is still no known mammalian NMN, NR or Nam de-amidase. Alternatively, the deamidation observed here could be due to bacterial contamination (Shats et al., 2020) of primary hepatocyte culture, or from non-enzymatic means.

By using our strategically designed NMN isotopes (Fig. 2) in vivo, we provide evidence for the deamidation of orally delivered NMN by the gut microbiome (Fig. 7). This finding is similar to the concept recently shown by Shats and colleagues, who demonstrated a role for bacterial interactions in mammalian $\mathrm{NAD}^{+}$homeostasis through the deamidation of Nam into $\mathrm{Na}$ by the bacterial enzyme PncA (Shats et al., 2020). In agreement with our study, this paper showed that antibiotic treatment increased the uptake of labelled nicotinamide in the colon, small intestine and liver, though a control group of animals treated with antibiotics alone were not used. Similarly, endogenous NMN levels were increased in the colon and endogenous NR was increased in the portal blood of germ-free mice, though no increase in NAD levels was observed with antibiotic treatment, as observed here. This may have been due to differences in tissue sampling or in the antibiotic cocktail used to ablate the microbiome in these studies, or the use of independently sampled sections of the small intestine and the colon, compared to our use of a pooled, heterogenous sample of the entire GIT.

While intravenous delivery of NMN results in intact assimilation into peripheral tissues such as the liver, kidney, brain and muscle, oral delivery of NMN results in cleavage at the glycosidic bond into free nicotinamide (Liu et al., 2018). Our results were in close alignment with those findings (Liu et al., 2018), where the ratio of intact $\mathrm{M}+7$ or $\mathrm{M}+6$ to $\mathrm{M}+0$ unlabelled $\mathrm{NAD}^{+}$was around $2 \%$, whereas the ratio of $\mathrm{M}+2$ labelled to $\mathrm{M}+0$ unlabelled $\mathrm{NAD}^{+}$, presumably as a result of incorporation of free Nam, was over 10\% (Fig. 5c, j, 6c, j). Given this evidence for the decomposition of NMN into free Nam prior to its uptake, a key question is why "later" precursors in $\mathrm{NAD}^{+}$synthesis such as NMN 
and NR appear to have different biological effects to Nam alone. A surprising aspect of these results was that treatment with $100 \%$ labelled NMN led to a striking increase in unlabelled NAD metabolites. In the case of both NR and NaR, treatment with NMN1 $(\mathrm{M}+6)$ or NMN2 $(\mathrm{M}+7)$ led to a stark increase in endogenous (M+0) levels (Fig. 5b, f, 6b, f, Supp. Fig. 4b, f, 6b, f). Endogenous (i.e. unlabelled, $\mathrm{M}+0$ ) levels of other metabolites were also increased by treatment with labelled NMN in both antibiotics treated and untreated animals, though not to the same degree as NR, which was elevated by over an order of magnitude. We also observed the increase in liver NaAD levels (Fig. 1f, 5g, n, $6 \mathrm{~g}, \mathrm{n}$ ) that was previously reported with exogenous NR treatment (Trammell et al., 2016), however the majority of this change was from unlabelled NaAD. These results were similar to those of Trammel et al, where isotope tracing showed that over half of the increase in NaAD levels following NR treatment were from $\mathrm{NaAD}$ that did not incorporate labels from exogenous NR, with only $8 \%$ of the NaAD pool incorporating isotopes from double-labelled NR. In contrast to that study, we argue that these counter-intuitive findings run against the classic mass-balance model, whereby it is assumed that exogenous $\mathrm{NAD}^{+}$precursors raise $\mathrm{NAD}^{+}$levels through their direct incorporation into the NAD metabolome. Instead, these results raise the idea that treatment with these exogenous precursors could indirectly trigger endogenous $\mathrm{NAD}^{+}$biosynthesis. The mechanism for this is not yet clear, though given the profound effect of antibiotic treatment, in particular for the overwhelming abundance of NR in the gut (Fig. 5b, 6b, Supp. Fig. 4b, 6b), are likely to involve interplay with the gut microbiome. Importantly, the increased production of endogenous $\mathrm{NAD}^{+}$metabolites following exogenous NMN/NR treatment suggests that the benefits of exogenous treatment with NMN or NR could be from indirect signalling, rather than direct incorporation - a finding that has profound importance for therapeutic strategies, in particular the choice of dosing. An important question regarding NR in particular is how its endogenous production can be increased. NR is available from dietary sources (Bieganowski and Brenner, 2004), and is an intermediate in the uptake of extracellular NMN (Belenky et al., 2009; Ratajczak et al., 2016b). NMN accumulation in neurons can trigger cell death through the NADase SARM1 (Gerdts et al., 2015), and disposal of NMN through its 
adenylation into $\mathrm{NAD}^{+}$can protect against neuronal death (Sasaki et al., 2016). It is possible that exogenous NMN triggers pathways that degrade endogenous NMN into NR, which could act as a back-up pool for NAD precursors, however the NMN ectonucleotidase that carries this out sits on the extracellular face of the plasma membrane (Belenky et al., 2009; Ratajczak et al., 2016b) rather than the cytosol, and the molar quantity of unlabelled NR that we observed in the gut relative to other metabolites including NMN cannot account for this. NAD ${ }^{+}$homeostasis is tightly maintained within a defined range (Hara et al., 2019), and it is possible that a bolus of exogenous NAD precursors trigger the degradation of existing $\mathrm{NAD}^{+}$to avoid exceeding this range, however an enzyme that would cleave $\mathrm{NAD}^{+}$to yield NR has not been identified. Another possibility is that rather degrading downstream metabolites into NR, exogenous NMN or NR could trigger a currently undescribed step in mammals that leads to endogenous NR production, suggesting that NR is not merely an exogenous nutrient or intermediate in extracellular NMN uptake. This observation may explain why NR and NMN treatment lead to biological and therapeutic effects that differ from Nam alone, despite their rapid metabolism into free Nam by the liver (Liu et al., 2018) - an observation that was in close alignment our results. We propose the existence of a signalling pathway in the GIT that is sensitive to both exogenous NAD precursors and to microbial metabolites, which can mediate endogenous NAD metabolism. Metabolite sensing members of the G-protein coupled receptor (GPCR) family are obvious candidates for this role, and could include the GPR109a, which acts as a receptor both for nicotinic acid and for butyrate, released from the microbial fermentation of dietary fibre - thus linking the observations of the deamidation of orally derived NAD precursors into their acid equivalents, a role for microbiome depletion in triggering the production of endogenous NAD metabolites, and poor evidence for the incorporation of intact NR or NMN into the $\mathrm{NAD}^{+}$metabolome.

\section{Study limitations}

To consolidate the hypothesis that the gut microbiome plays a role in $\mathrm{NAD}^{+}$homeostasis, future studies should measure isotope labelling of $\mathrm{NAD}^{+}$metabolites in faecal contents of mice to determine 
whether these compounds are being utilised by the microbiome, rather than being excreted via other mechanisms. Further, to rule out a role for the effects of antibiotic treatment on host tissues rather than depletion of the gut microbiome, future studies could use treatment in antibiotic treated animals in which the microbiome has been reconstituted. Regarding the interpretation of indirect transport predominating over direct NMN transport, important limitations are that intestinal segments were combined, causing a loss of functional heterogeneity, including sections of the jejunum and ileum recently reported to express the putative NMN transporter SLC12A8 (Grozio et al., 2019). Further, limited availability of isotope labelled material meant that this study used a single time point, rather than a time course which also encompassed very early timepoints, possibly missing the minute-order kinetics of direct NMN transport, as previously reported (Mills et al., 2016; Yoshino et al., 2011). Finally, one of the most important aspects of this work was the observation that treatment with labelled NMN led to an increase in levels of unlabelled NR, NaR and other metabolites. Further work is needed to understand how this occurs, for example, whether it is due to the acute up-regulation of $\mathrm{NAD}^{+}$biosynthetic enzymes, the time-scale by which this increase occurs, and a direct comparison of different isotope labelled $\mathrm{NAD}^{+}$precursors to identify which metabolites trigger the production of endogenous metabolites under normal circumstances and during depletion of the microbiome. Finally, we propose the existence of cell surface receptors and downstream signalling pathways in the gut that are sensitive to both microbial metabolites and exogenous $\mathrm{NAD}^{+}$precursors - identifying these will be a key goal of testing our hypothesis around the role of exogenous metabolites in endogenous $\mathrm{NAD}^{+}$metabolism. 


\section{STAR Methods}

STAR methods are available in Supplementary Material and on our Mendeley data site.

\section{Acknowledgements}

Funding was from the National Health and Medical Research (NHMRC) of Australia as a Career Development Fellowship APP1122484 to LEW, and sponsored research from Jumpstart Fertility. We wish to thank anonymous donors for philanthropic support.

\section{Author contributions}

LJK conducted experiments, analysed data, prepared figures, wrote manuscript. TJC conducted microbiome analyses. EWKP, TTC, JW prepared isotope labelled NMN. SPT and DAS provided critical feedback and interpretation. LEQ conducted experiments, extracted and analysed data, wrote manuscript. LEW conceived of and designed study, obtained funding, supervised experiments, analysed data, prepared figures, wrote manuscript.

\section{Declaration of interests}

EWKP and JW are employees and shareholders of GeneHarbor Biotechnologies. SPT is the CEO of Jumpstart Fertility, which is developing NAD ${ }^{+}$raising compounds for therapeutic use. LEW and DAS are co-founders, shareholders, directors and advisors of Jumpstart Fertility and the Life Biosciences group which includes Jumpstart Fertility, Continuum Biosciences, Senolytic Therapeutics, Selphagy, and Animal Biosciences. LEW and DAS are also advisors to and shareholders in the EdenRoc group of companies, which includes Metro Biotech NSW and Metro International Biotech, Arc-Bio, Dovetail Genomics, Claret, Revere Biosciences, and Liberty Biosecurity. LEW is an advisor and shareholder in Intravital Pty Ltd. DAS is an inventor on a patent application that has been licensed to Elysium Health. Updated affiliation are at https://genetics.med.harvard.edu/sinclairtest/people/sinclair-other.php. 


\section{Figure 1}
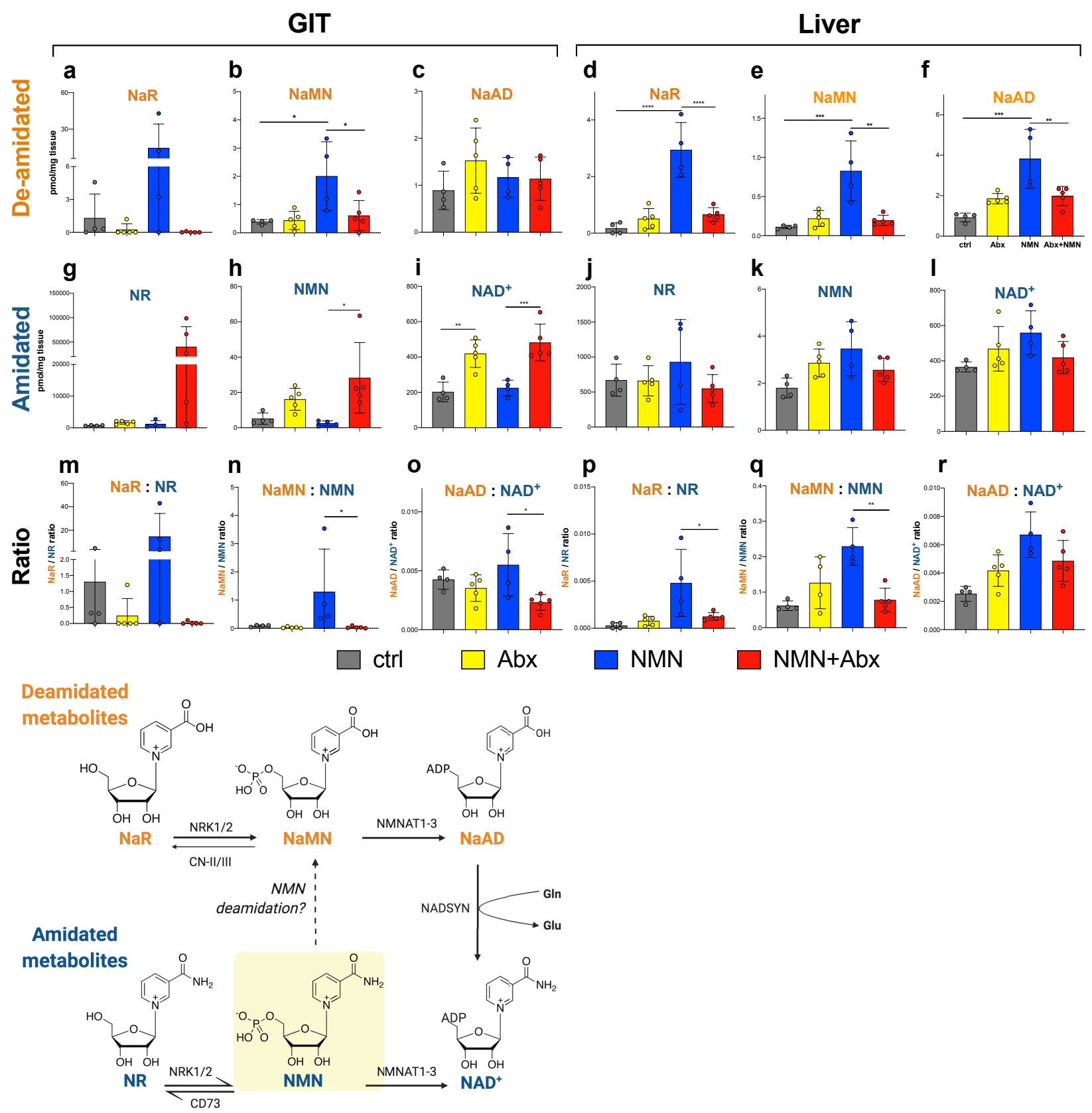

Figure 1. NMN treatment leads to the microbiome-dependent formation of deamidated NAD ${ }^{+}$

metabolites in vivo. Mice treated with antibiotics $(\mathrm{Abx})$ to ablate the gut microbiome were administered a single dose of unlabelled nicotinamide mononucleotide (NMN) $(500 \mathrm{mg} / \mathrm{kg}$, oral gavage). Gastrointestinal tissue (GIT) (a-c) and liver tissue (d-f) were subject to targeted mass spectrometry to quantify the deamidated metabolites (a-f) nicotinic acid riboside (NaR) (a, d), nicotinic acid mononucleotide $(\mathrm{NaMN})(\mathrm{b}, \mathrm{e})$ and nicotinic acid adenine dinucleotide (NAAD) (c, f), as well as their amidated counterparts (g-l) nicotinamide riboside (NR) (g, j), NMN (h, k) and nicotinamide adenine dinucleotide $\left(\mathrm{NAD}^{+}\right)(\mathrm{i}, 1)$. These data were then expressed as ratios between 
bioRxiv preprint doi: https://doi.org/10.1101/2020.09.10.289561; this version posted September 11, 2020. The copyright holder for this preprint (which was not certified by peer review) is the author/funder, who has granted bioRxiv a license to display the preprint in perpetuity. It is made available under aCC-BY-ND 4.0 International license.

de-amidated and amidated counterparts in GIT (m-o) and liver (p-r). Data analysed by 2-way ANOVA with Sidak's post-hoc test, exact p-values and F values in supplementary files. $N=4-5$ animals per group, ${ }^{*} \mathrm{p}<0.05, * * \mathrm{p}<0.01, * * * \mathrm{p}<0.001, * * * * \mathrm{p}<0.0001$ 


\section{Figure 2}

a

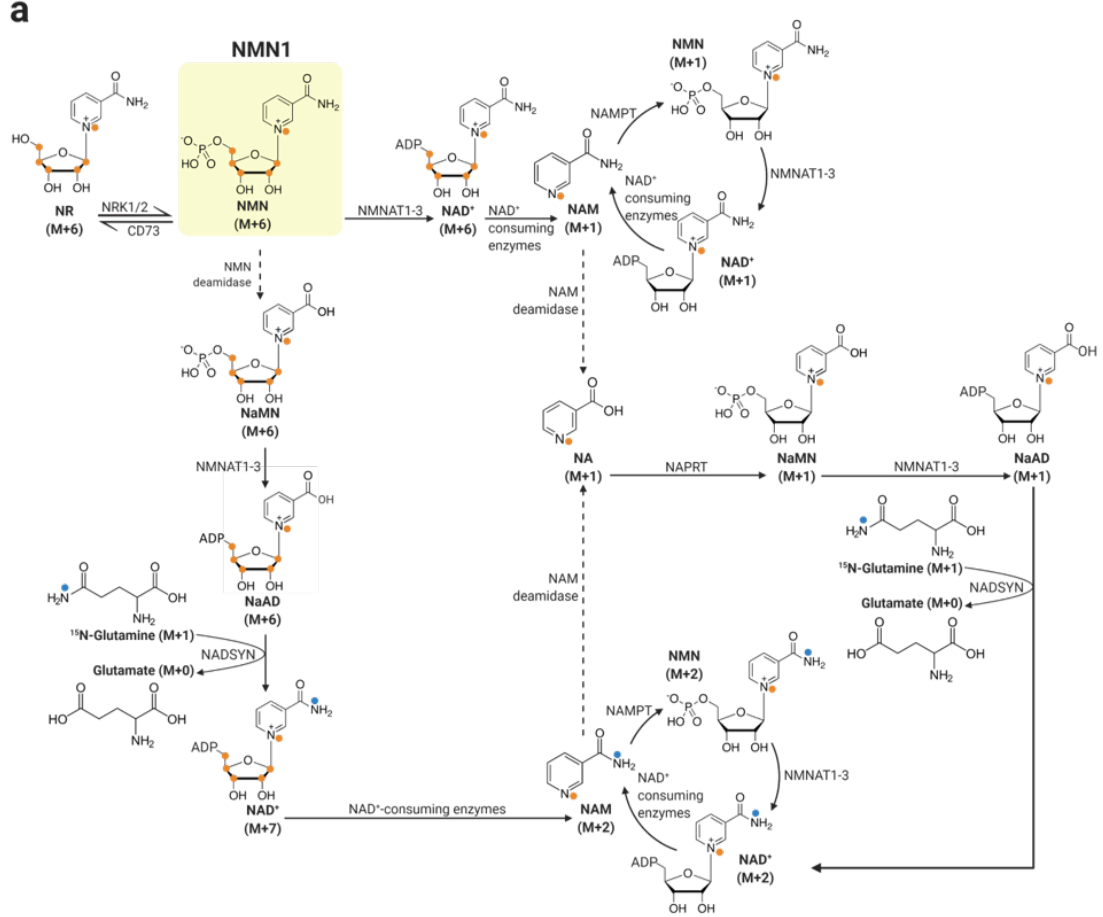

b

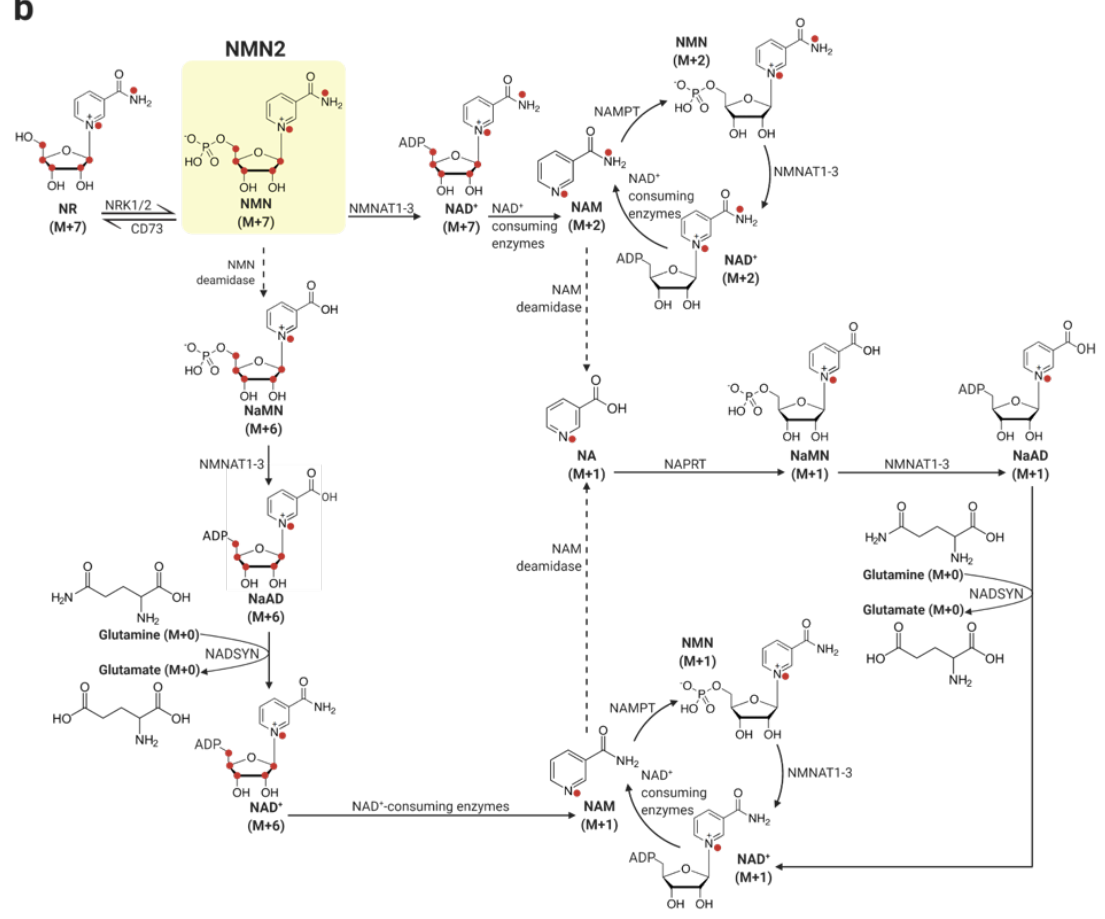

Figure 2. Scheme for assimilation of isotope labelled NMN into the $\mathrm{NAD}^{+}$metabolome.

$\mathrm{NMN}$ (box on left) was labelled with ${ }^{13} \mathrm{C}$ at all 5 carbon positions of the ribose moiety (highlighted in red) and with ${ }^{15} \mathrm{~N}$ at the base (highlighted in blue) and amine (highlighted in orange) of the nicotinamide moiety for a total mass shift of $\mathrm{M}+7$. The expected mass shift for each species is shown, with steps catalyzed by mammalian enzymes in solid black lines, and steps that are only known to be carried out by bacterial enzymes shown with dashed lines. 


\section{Figure 3}
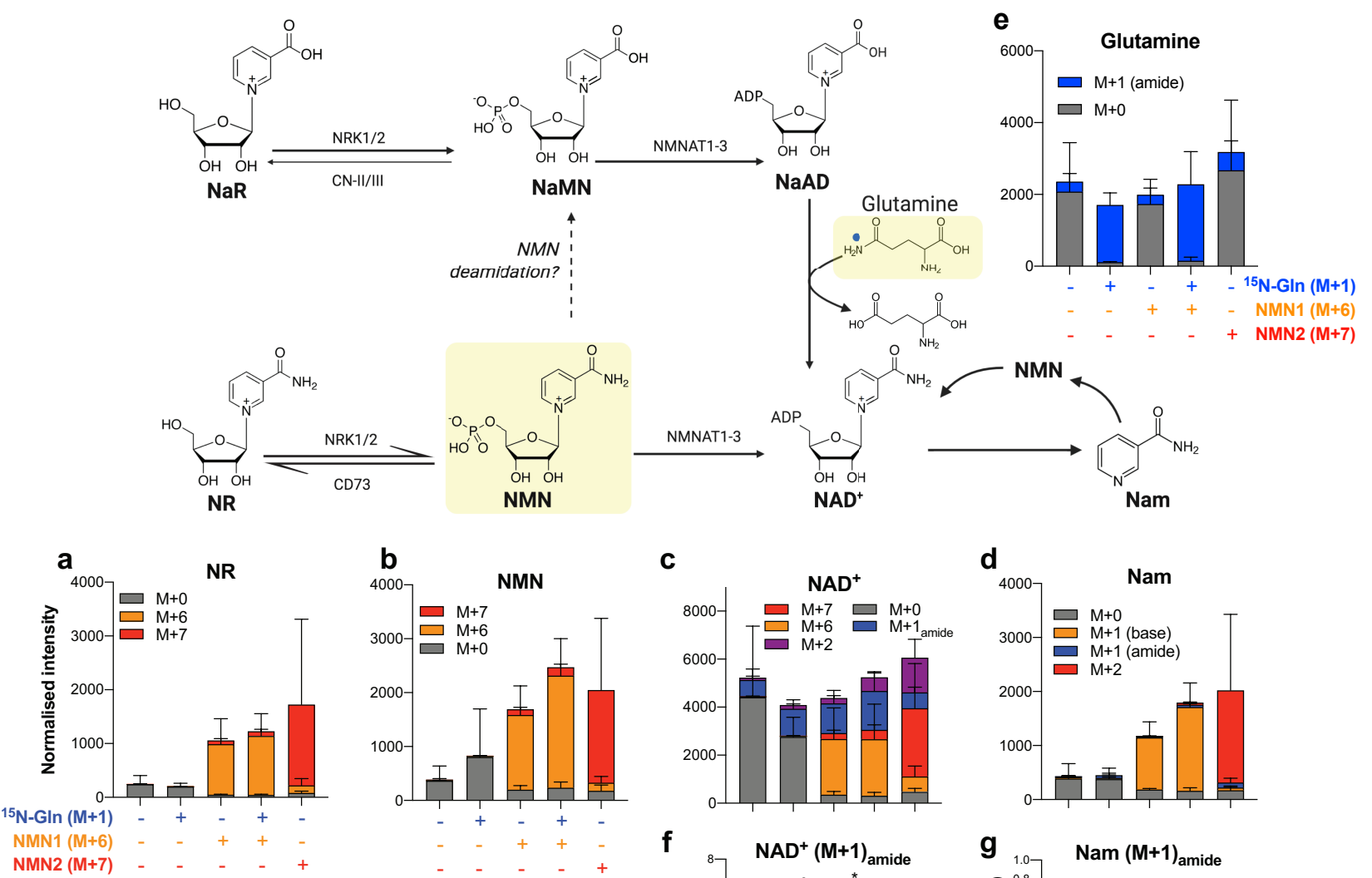

C
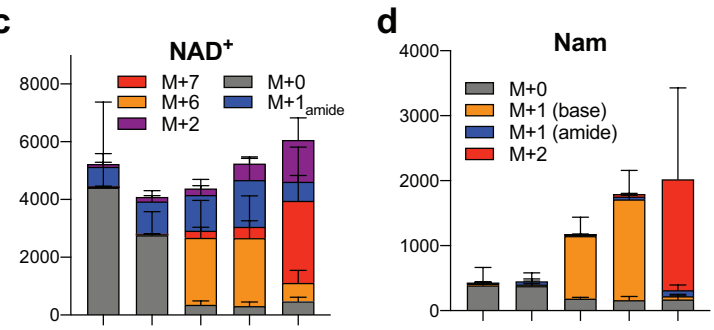

NMN2 (M+7)
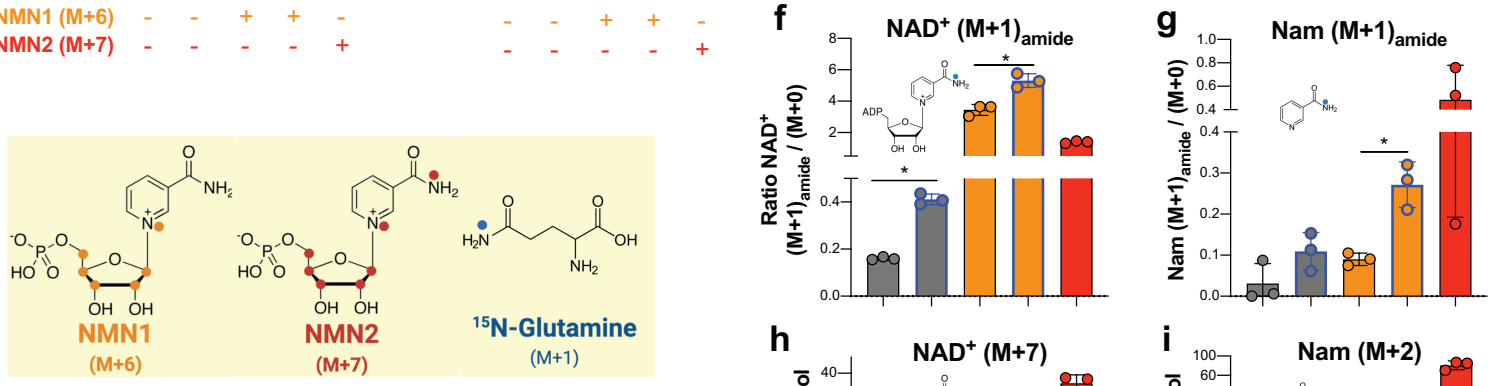

h
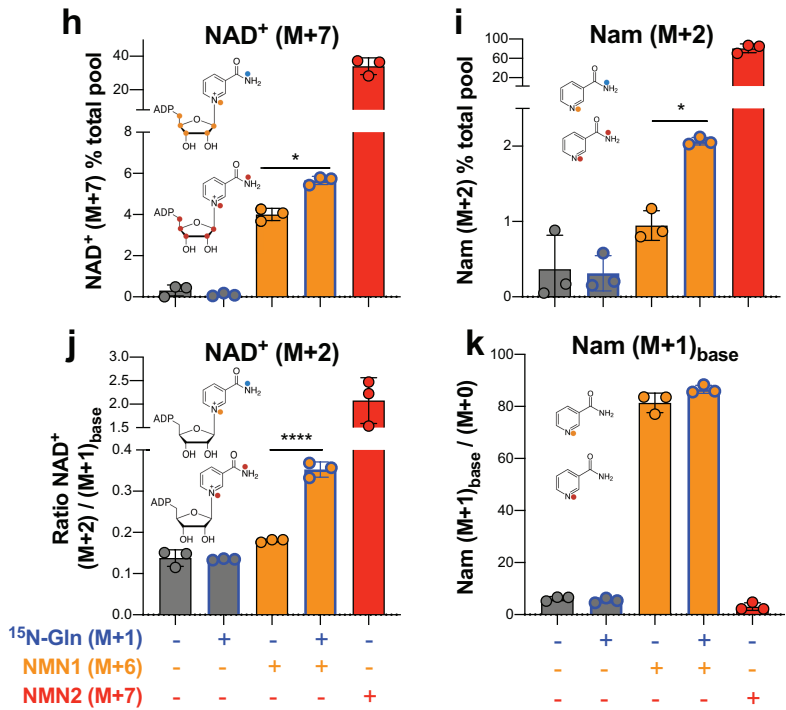

Figure 3. ${ }^{15} \mathrm{~N}$-glutamine labelling of $\mathrm{NAD}^{+}$synthesis. Primary hepatocytes were treated with NMN1 $(\mathrm{M}+6)$ or NMN2 $(\mathrm{M}+7)$ isotopes $(200 \mu \mathrm{M})$ in the presence of unlabelled or amide labelled ${ }^{15} \mathrm{~N}$-glutamine $(\mathrm{M}+1)(4 \mathrm{mM})$ for $24 \mathrm{hr}$, to measure the degree of NAD synthesis and the incorporation of exogenous NMN via the de novo pathway, which incorporates the amide label of 
${ }^{15} \mathrm{~N}-\mathrm{G} \ln$ through the enzyme NAD synthetase (NADS). Exogenous NMN1 and NMN2 isotopes in the presence of ${ }^{15} \mathrm{~N}-\mathrm{G}$ ln led to the expected isotopic labelling of a) NR, b) NMN, c) $\mathrm{NAD}^{+}$d) Nam and e) Gln. Ratios of f) $\mathrm{M}+1_{\text {amide }}$ labelled to unlabelled NAD, g) $\mathrm{M}+7$ labelled NAD,$\left.+ \mathrm{h}\right) \mathrm{M}+2$ labelled NAD + and i) $\mathrm{M}+1_{\text {amide }}$ Nam were consistent with labelling by ${ }^{15} \mathrm{~N}-\mathrm{Gln}$, with no change in $\mathrm{j}$ ) $\mathrm{M}+1_{\text {base }}$ labelled Nam acting as a negative control, as no change in labelling at the ring position is expected. This resulted in 1) M+2 labelled Nam during NMN1 and 15N-Gln treatment. Data analysed by 2-way ANOVA with Sidak's post-hoc test, $\mathrm{n}=3$ biological replicates. 


\section{Figure 4}

\section{Supernatant}
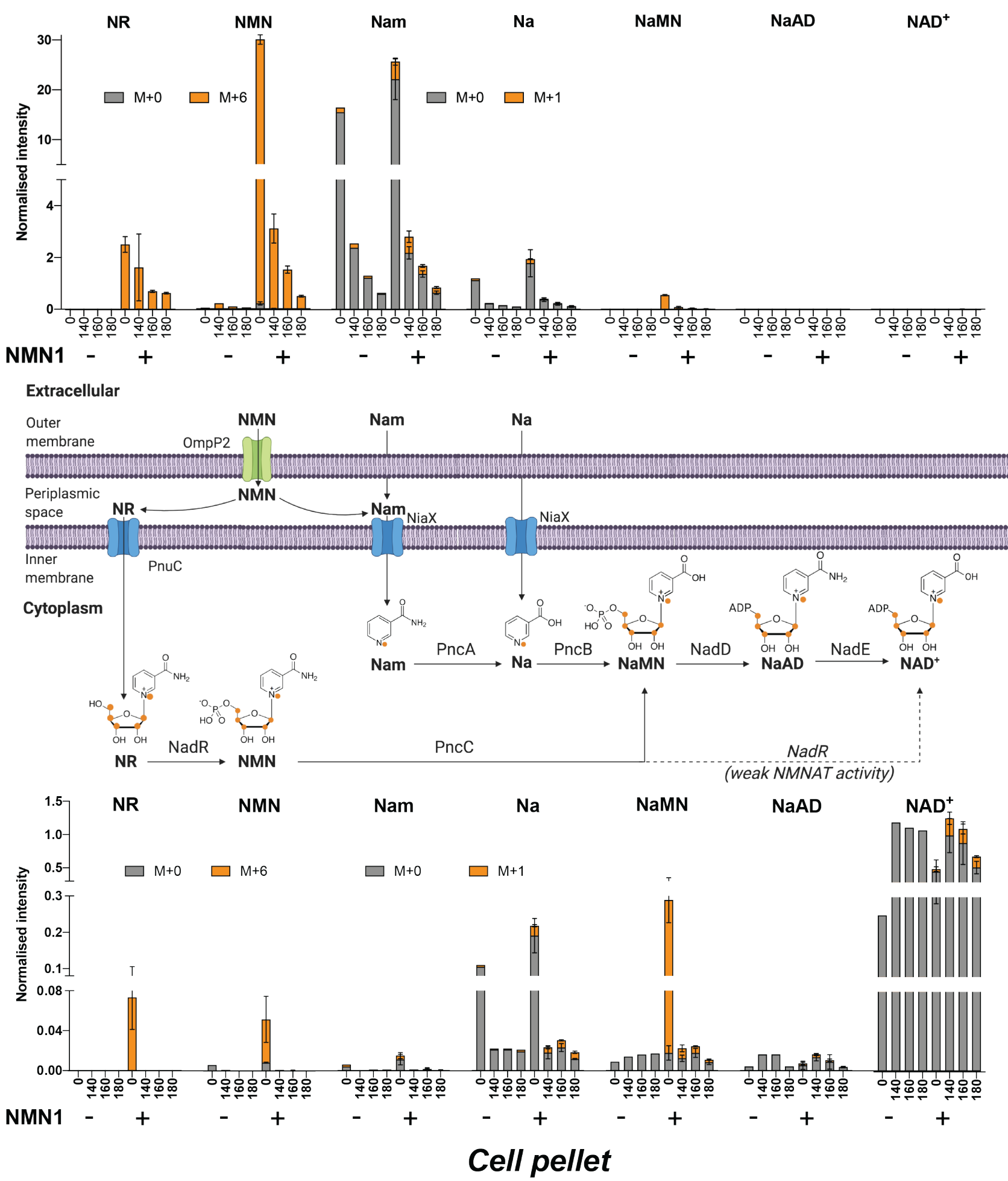

Figure 4. NMN deamidation in bacteria. Liquid cultures of E. coli OP50 bacteria were supplemented with $\mathrm{M}+6$ labelled NMN1 $(0.1 \mathrm{mM})$ at inoculation of a fresh culture. Samples were taken at time 0 (after NMN), 140, 160 and 180 minutes after NMN supplementation. Following separation of the culture supernatant (top) from the cell lysate (bottom), metabolites were extracted and subjected to targeted LC-MS/MS mass spectrometry to detect the incorporation of the M+6 
bioRxiv preprint doi: https://doi.org/10.1101/2020.09.10.289561; this version posted September 11, 2020. The copyright holder for this preprint (which was not certified by peer review) is the author/funder, who has granted bioRxiv a license to display the preprint in perpetuity. It is made available under aCC-BY-ND 4.0 International license.

isotope label into $\mathrm{NMN}, \mathrm{NR}, \mathrm{NaMN}$ and $\mathrm{NAD}^{+}$as well as $\mathrm{M}+1$ labelling of nicotinamide (Nam) and nicotinic acid ( $\mathrm{Na}$ ) in both the culture supernatant (top) and cell lysates (bottom). Data represents mean \pm s.d. ( $\mathrm{n}=3-5$ samples per time point). 


\section{Figure 5}

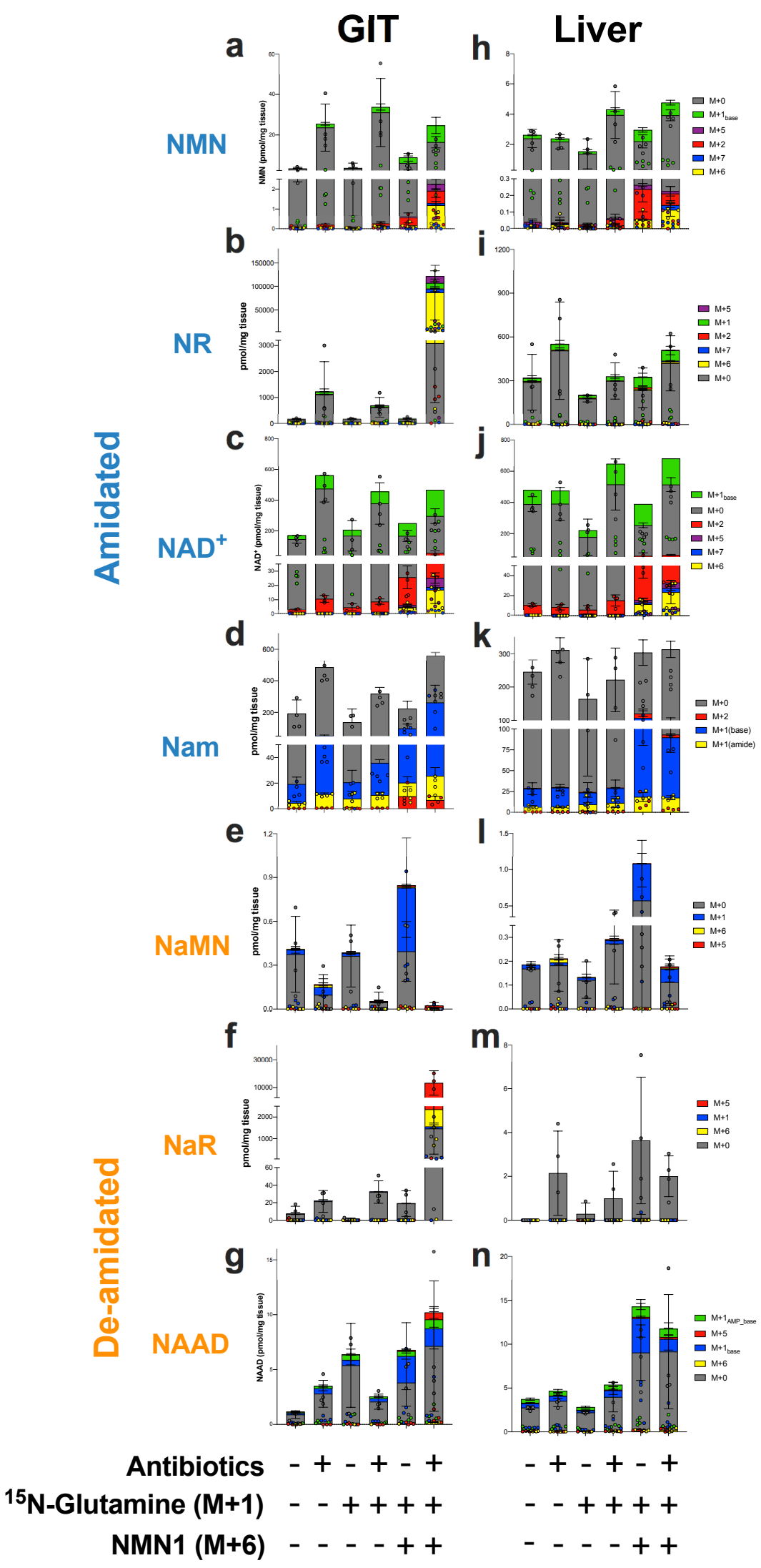

Figure 5. Incorporation of isotope labelled NMN into the NAD metabolome in the GIT. Animals were treated with antibiotics (Abx) to deplete the microbiome, followed by an oral gavage $(50 \mathrm{mg} / \mathrm{kg})$ 
of NMN1 (M+6) with adjacent i.p. administration of ${ }^{15} \mathrm{~N}-\mathrm{G} \ln (735 \mathrm{mg} / \mathrm{kg}, 10 \mathrm{ml} / \mathrm{kg}$ body weight). Four hr later, GIT (a-g) and liver (h-n) were rapidly preserved for targeted metabolomics analysis to identify labelling of $(a, h)$ NMN, (b, i) NR, (c, j) NAD,$+(d, k)$ Nam, (e, l) NaMN, (f, m) NaR and (g, n) NaAD. n=3-4 animals per group, data presented as stacked bars of each isotopologue, with raw data points for each isotopologue overlaid on bar charts. 
Figure 6

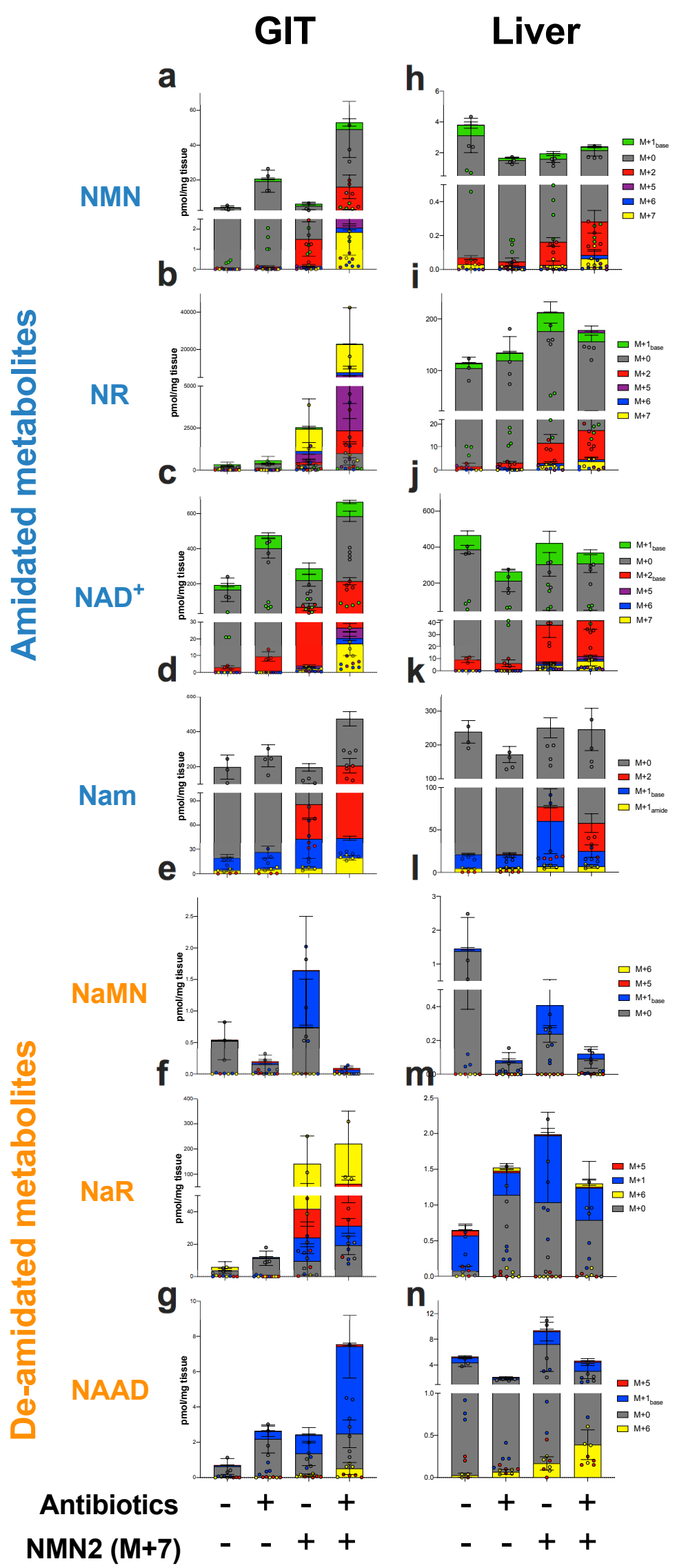

Figure 6. Incorporation of isotope labelled NMN2 $(M+7)$ into the NAD metabolome. Animals were treated with antibiotics (Abx) to deplete the microbiome, followed by an oral gavage $(50 \mathrm{mg} / \mathrm{kg})$ 
of NMN2 (M+7). Four hr later, GIT (a-g) and liver (h-n) were rapidly preserved for targeted metabolomics analysis to identify labelling of (a, h) NMN, (b, i) NR, (c, j) NAD ${ }^{+},(d, k)$ Nam, (e, l) NaMN, (f, m) NaR and (g, n) NaAD. n=3-4 animals per group, data presented as stacked bars of each isotopologue, with raw data points for each isotopologue overlaid on bar charts. 


\section{Figure 7}

a

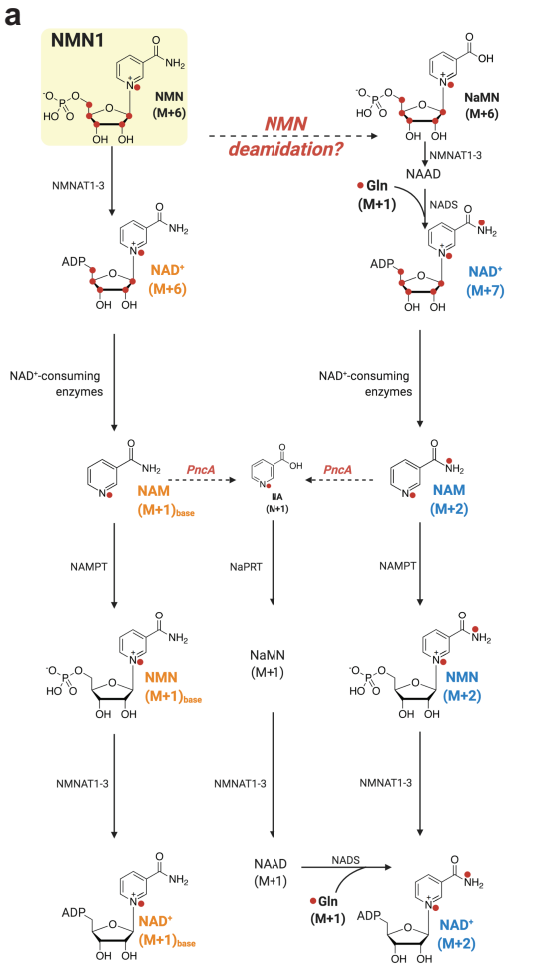

Amidated route

De-amidated route

j
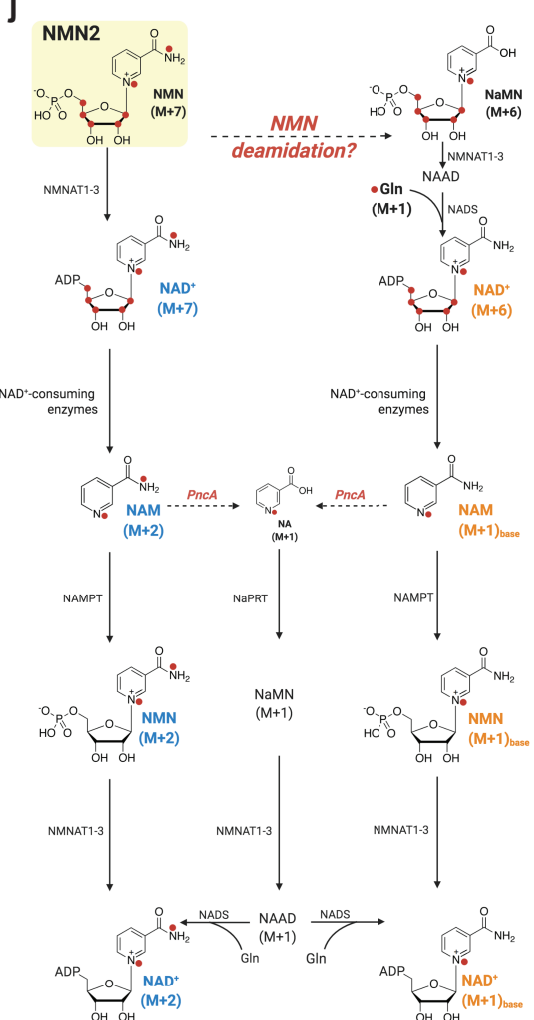

De-amidated route
NMN1 $(M+6)+{ }^{15} \mathrm{~N}-\mathrm{GIn}(\mathrm{M}+1)$

GIT

Liver

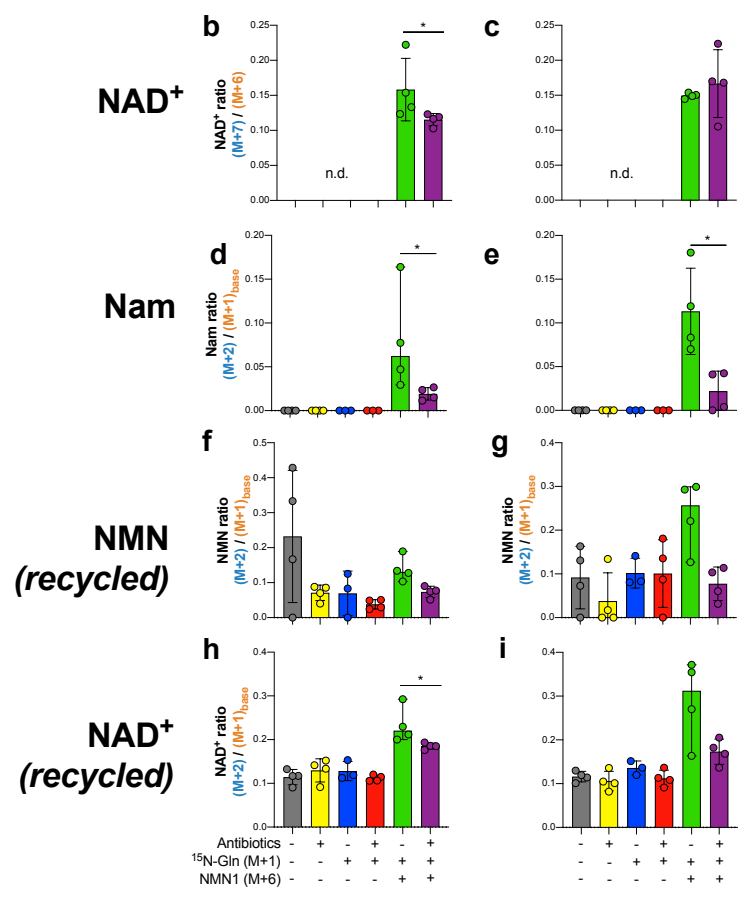

NMN2 (M+7)

$\mathrm{NAD}^{+}$

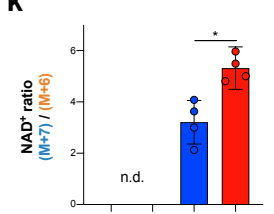

m

Nam
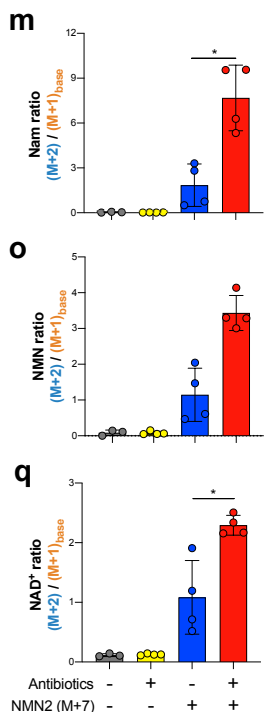
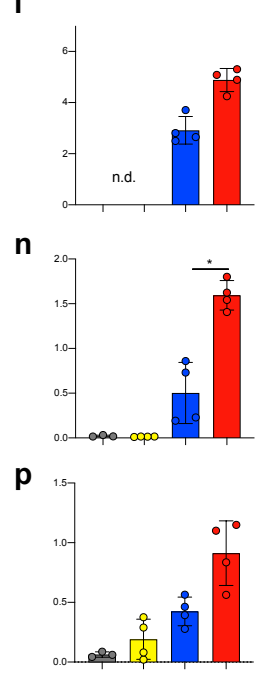

n
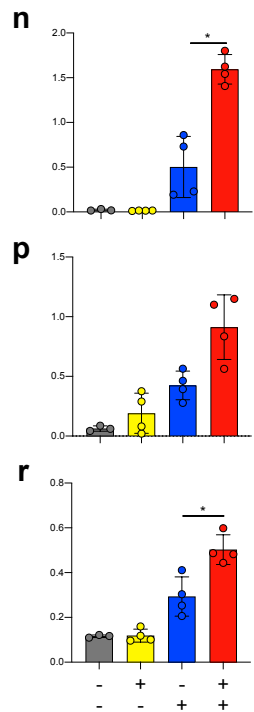

Figure 7. Contribution of the microbiome to NMN deamidation. Antibiotic treated animals were orally administered with the a) NMN1 $(\mathrm{M}+6)$ isotope in the presence or absence of ${ }^{15} \mathrm{~N}$-glutamine $(\mathrm{M}+1)$, with the formation of $\mathrm{M}+7$ labelled $\mathrm{NAD}+$ or $\mathrm{M}+2$ labelled Nam reflective of incorporation 
following deamidation and reamidation by the enzyme NADS. Antibiotic treatment reduced (b, c) $\mathrm{M}+2$ labelling of nicotinamide (Nam), (d, e) M+7 labelling of $\mathrm{NAD}^{+}$and (f, g) $\mathrm{M}+2$ labelling of $\mathrm{NAD}^{+}$, which reflects the incorporation of recycled Nam. Data are expressed as ratios to $\mathrm{M}+1_{\text {base }}$ $\mathrm{NAM}$ and $\mathrm{M}+6$ or $\mathrm{M}+1 \mathrm{NAD}^{+}$, which are the expected isotope products of NMN or NR assimilation. Incorporation of this extra label was limited by the availability of ${ }^{15} \mathrm{~N}$-glutamine $(\mathrm{M}+1)$ as $\left.\mathrm{h}\right)$ a proportion of the endogenous glutamine pool. In a separate cohort, animals were treated with i) NMN2 $(\mathrm{M}+7)$, where loss of the amide ${ }^{15} \mathrm{~N}$ label to form $\mathrm{M}+6$ labelled $\mathrm{NAD}^{+}$or $\mathrm{M}+1_{\text {base }}$ labelled Nam would reflect deamidation. Antibiotic treatment protected $\mathrm{M}+2 \mathrm{NAM}(\mathrm{j}, \mathrm{k}), \mathrm{M}+7 \mathrm{NAD}^{+}(\mathrm{l}, \mathrm{m})$ and $\mathrm{M}+2 \mathrm{NAD}^{+}(\mathrm{n}, \mathrm{o})$ against loss of the NMN2 ${ }^{15} \mathrm{~N}$ amide compared to untreated animals. Tissues shown in the left column $(\mathrm{b}, \mathrm{d}, \mathrm{f}, \mathrm{j}, \mathrm{l}, \mathrm{n})$ are gastrointestinal tract $(\mathrm{GIT})$, on the right column (c, e, g, $\mathrm{k}, \mathrm{m}, \mathrm{o})$ are liver. NMN1 and NMN2 experiments were run in separate cohorts of animals, each measurement represents tissue from a separate animal. Comparisons of isotope treated groups with or without antibiotic treatment were analysed by Mann-Whitney U-test, ${ }^{*} \mathrm{p}=0.0286, \mathrm{n}=4$ animals per group. 


\section{Figure 8}

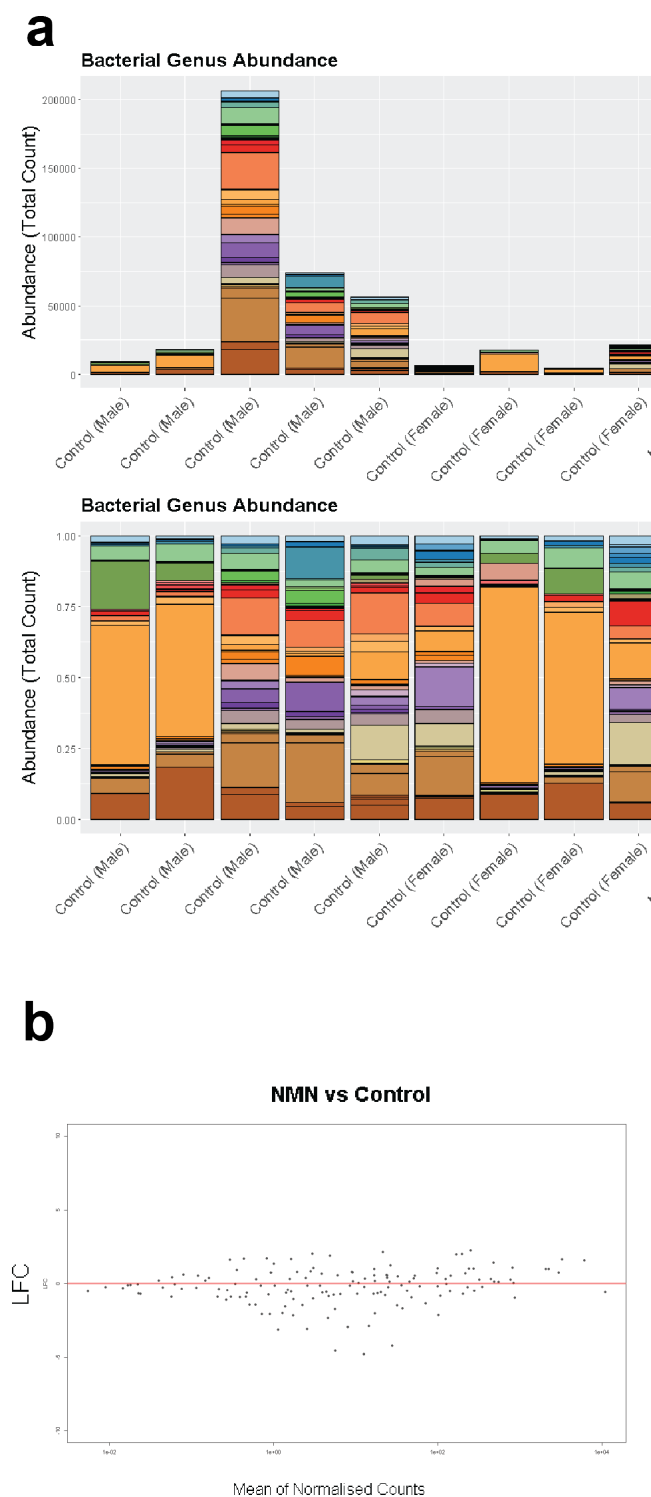

C

d
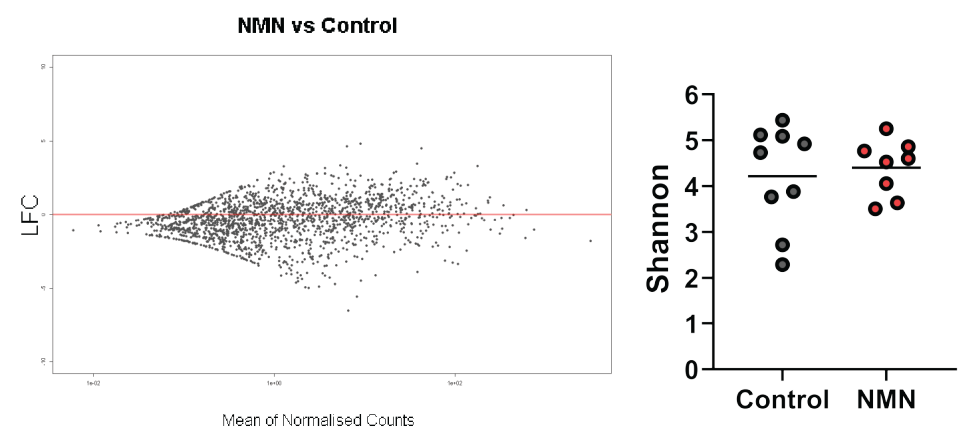

Figure 8. Chronic NMN treatment does not alter microbiome diversity. Aged (97-week old) male and female mice were treated with NMN through addition to drinking water ( $400 \mathrm{mg} / \mathrm{kg} / \mathrm{day})$ for 8 10 weeks prior to cull, and faecal microbiome samples subject to long-read 16S rRNA sequencing. (a) Stacked bar plots represent the total reads and relative abundance of bacterial taxa at the genus level for untreated or NMN treated animals, also shown as differential expression by the (b) genus level and (c) by operational taxonomic unit (OTU) level. There was no change in microbiome diversity, shown by the (d) Shannon alpha diversity index. 
bioRxiv preprint doi: https://doi.org/10.1101/2020.09.10.289561; this version posted September 11, 2020. The copyright holder for this preprint (which was not certified by peer review) is the author/funder, who has granted bioRxiv a license to display the preprint in perpetuity. It is made available under aCC-BY-ND 4.0 International license.

\section{SUPPLEMENTARY MATERIAL}




\section{Supplementary Figure 1}

a

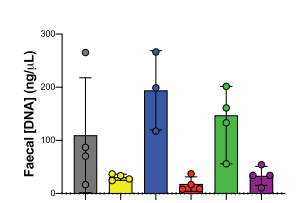

Antibiotics - +-++

${ }^{15} \mathrm{~N}-\mathrm{G} \ln (\mathrm{M}+1)-\quad++++$

NMN1 (M+6) - - - - +

C Bacterial Genus Abundance
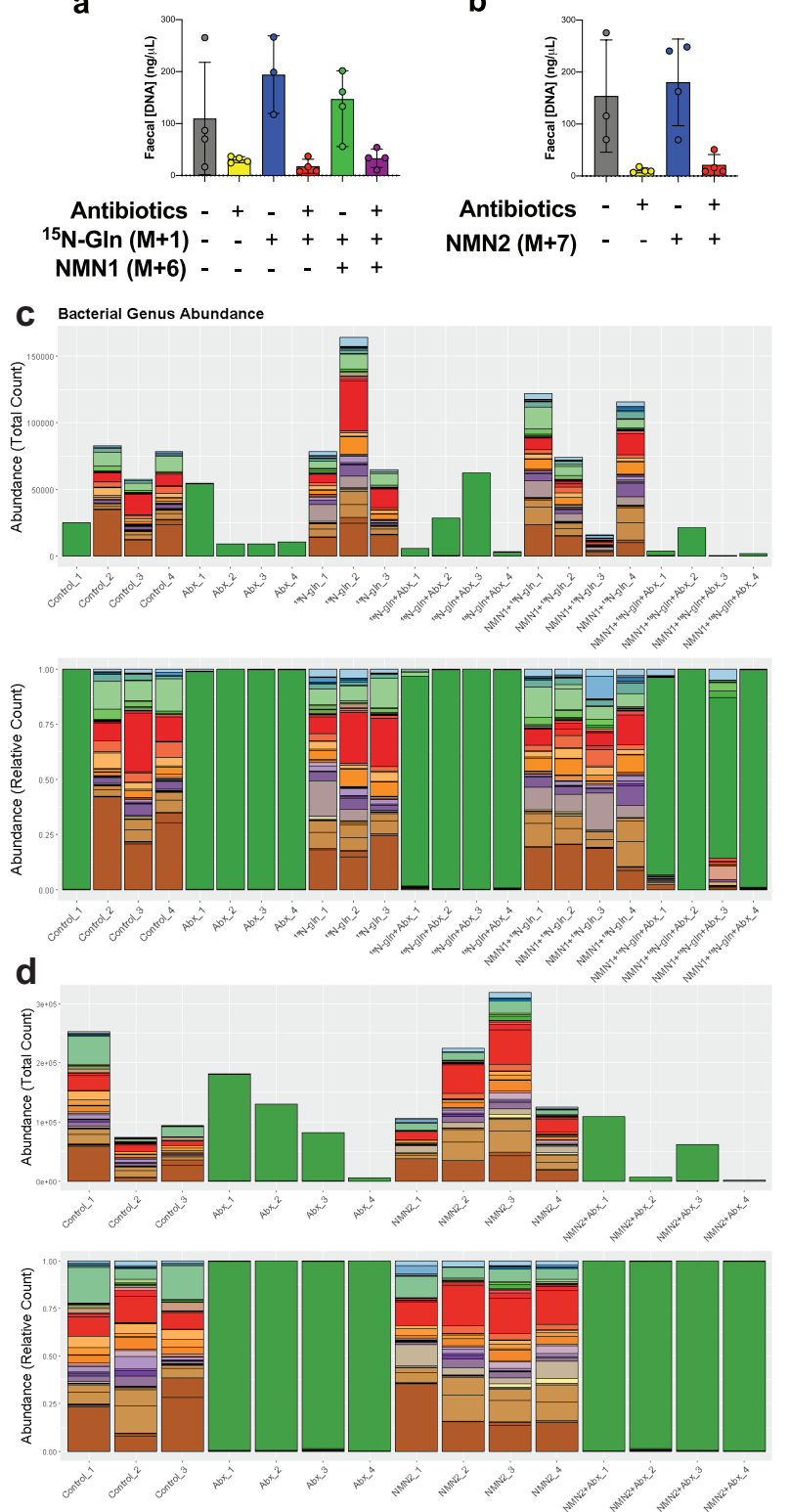

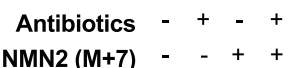

b
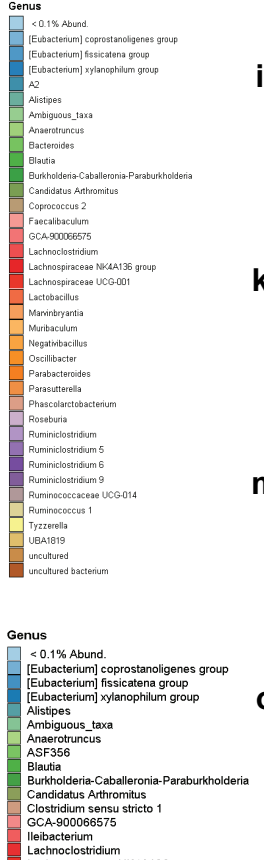

${ }^{15} \mathrm{~N}-\mathrm{G} \ln (\mathrm{M}+1)-{ }_{-}+++$
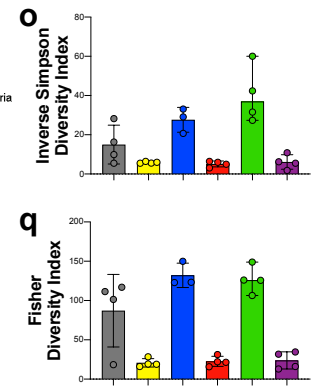

Abx - + - + +

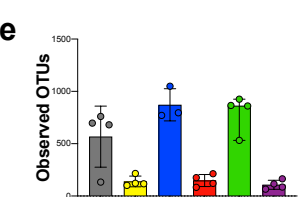

g

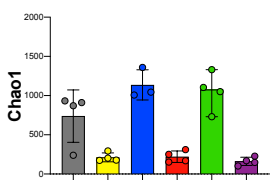

i

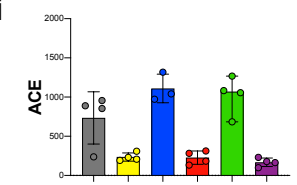

k

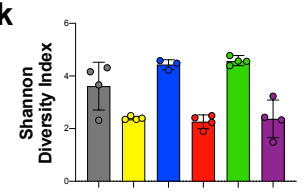

m

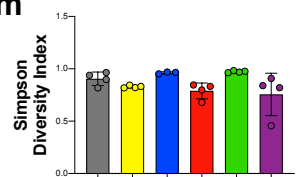

n

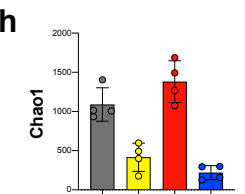

j

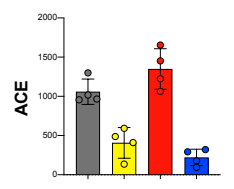

I
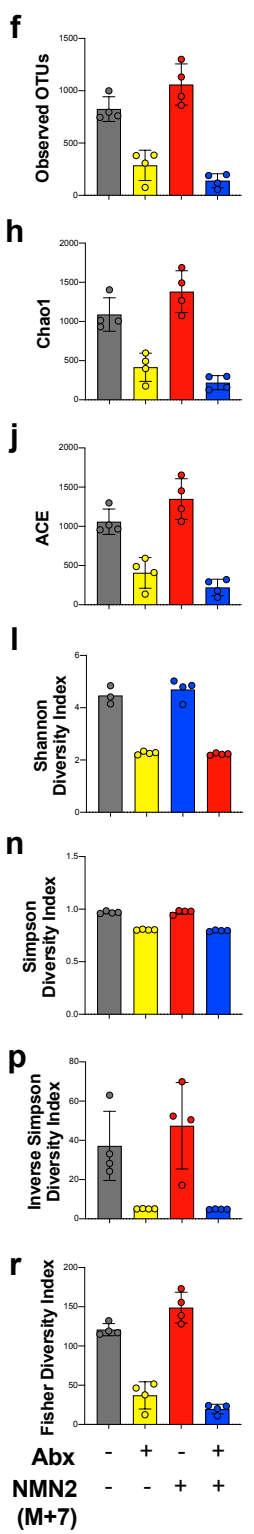

Supplementary Figure 1. Antibiotics treatment ablates the gut microbiome. Following antibiotic treatment in the NMN1 and NMN2 mouse cohorts, (a-b) DNA was extracted from faeces to measure changes in DNA concentration. Uniform amounts of DNA were then subject to (c-d) full-length $16 \mathrm{~S}$ rRNA Nanopore sequencing, with species abundance shown here at the genus level. Sequencing revealed a reduction in (e-f) operational taxonomic units (OTUs), the (g-h) Chaol and (i-j) ACE species richness indices, and the (k-1) Shannon, (m-n) Simpson, (o-p) Inverse Simpson and (q-r) Fisher diversity indices. Data shown are non-rarefied; rarefication showed identical results (data not shown). Each data point represents samples from a separate animal 


\section{Supplementary Figure 2}

a

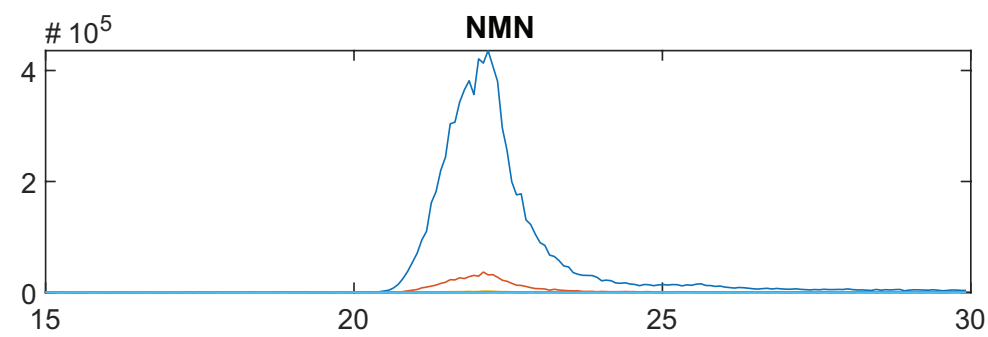

b

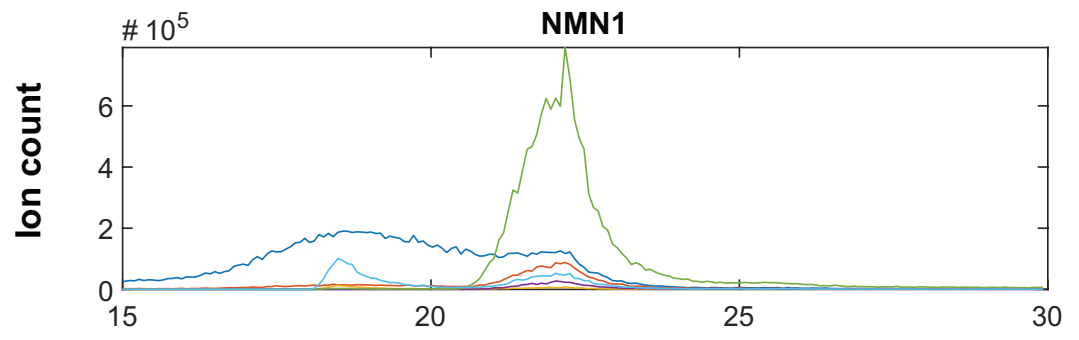

C

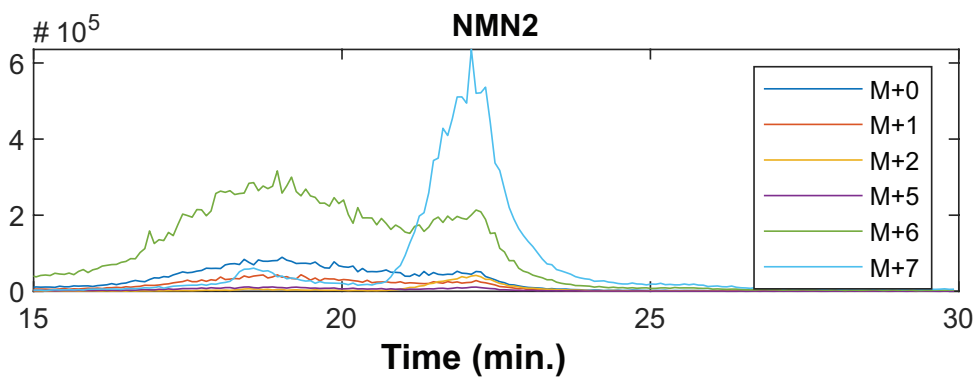

d
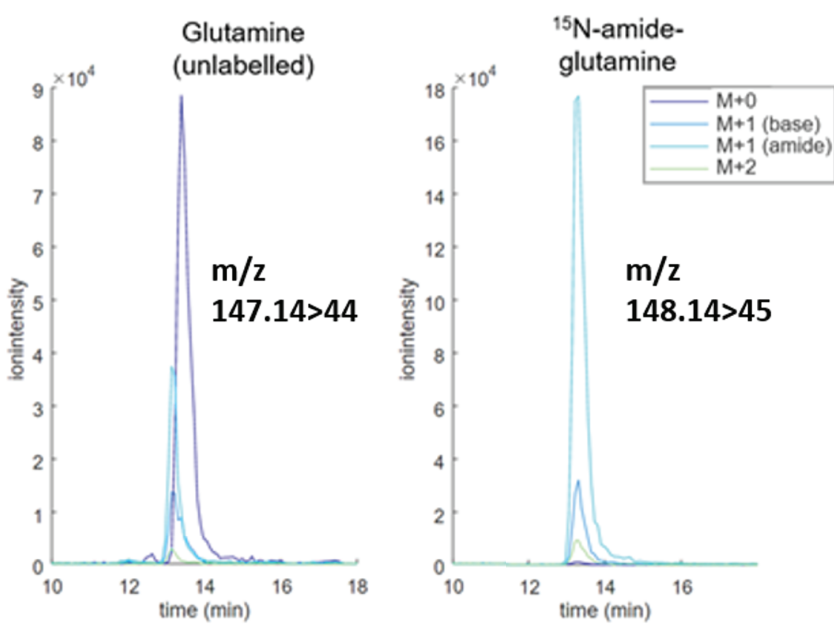

Supplementary Figure 2. Chromatogram of isotope labelled NMN and glutamine (GIn) using

MRM LC-MS/MS. The above chromatograms represent individual peaks (ion count) for a) $100 \mu \mathrm{M}$ unlabelled NMN in combined NAD metabolite standard curve mixture, b) M+6 labelled NMN1 and c) $\mathrm{M}+7$ labelled NMN2, as well as d) unlabelled and ${ }^{15} \mathrm{~N}$-amide labelled glutamine. 


\section{Supplementary Figure 3}

\section{Plasma}

a
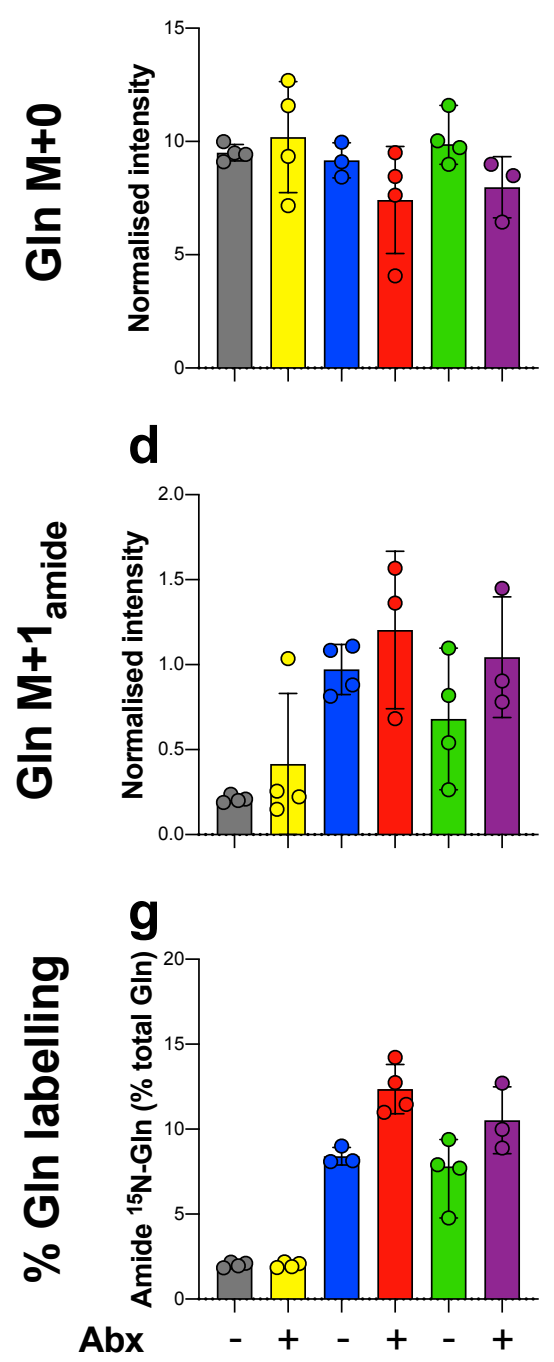

Liver

b

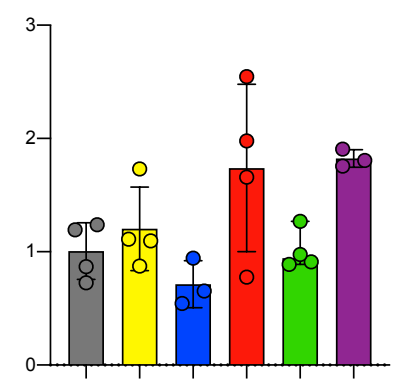

\section{e}

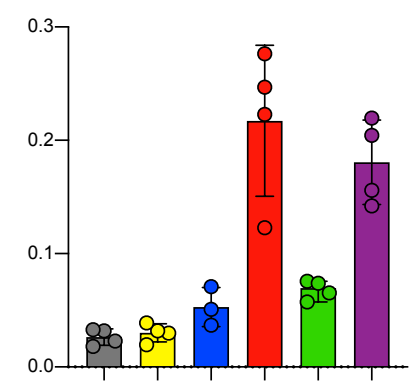

h

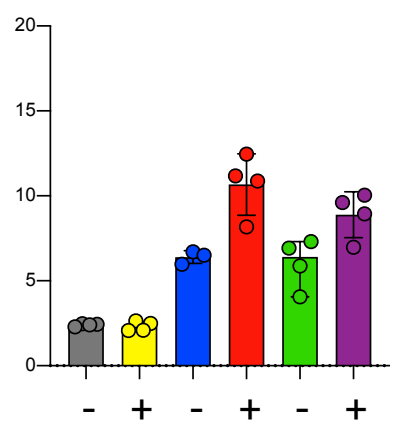

GIT
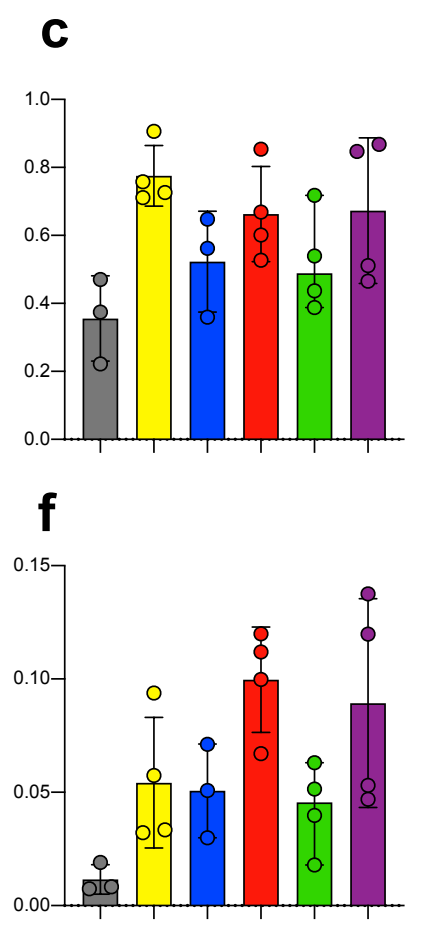

i

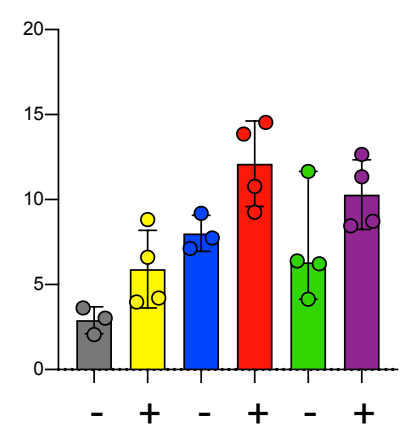

${ }^{15} \mathrm{~N}-\mathrm{G} \ln (\mathrm{M}+1)$

NMN1 (M+6)

Supplementary Figure 3. ${ }^{15} \mathrm{~N}-$ Glutamine labelling of tissues (Main Fig. 5). Animals were treated with antibiotics (Abx) to deplete the microbiome, followed by an oral gavage $(50 \mathrm{mg} / \mathrm{kg}$ ) of NMN1 $(\mathrm{M}+6)$ with adjacent i.p. administration of ${ }^{15} \mathrm{~N}-\mathrm{Gln}(735 \mathrm{mg} / \mathrm{kg}, 10 \mathrm{ml} / \mathrm{kg}$ body weight $)$. Four hr later, tissues were preserved for metabolomic analysis of $(\mathrm{a}-\mathrm{c})$ endogenous $(\mathrm{M}+0)$ and $(\mathrm{d}-\mathrm{e})$ amide labelled $\left(\mathrm{M}+1_{\text {amide }}\right) \mathrm{Gln}$, (g-i) expressed as a percentage ${ }^{15} \mathrm{~N}$ amide glutamine labelling of the overall Gln pool. Tissues subject to analysis are (a, d, g) plasma, (b, e, h) liver and (c, f, i) GIT. n=3-4 animals per group, each data point represents a separate animal, error bars are SD. 


\section{Supplementary Figure 4}

\section{Endogenous}

a

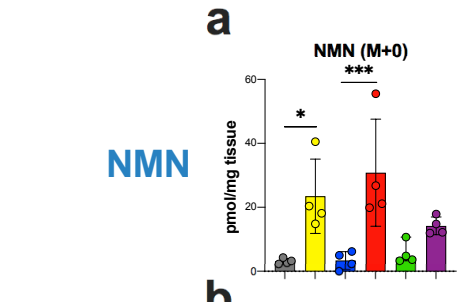

b

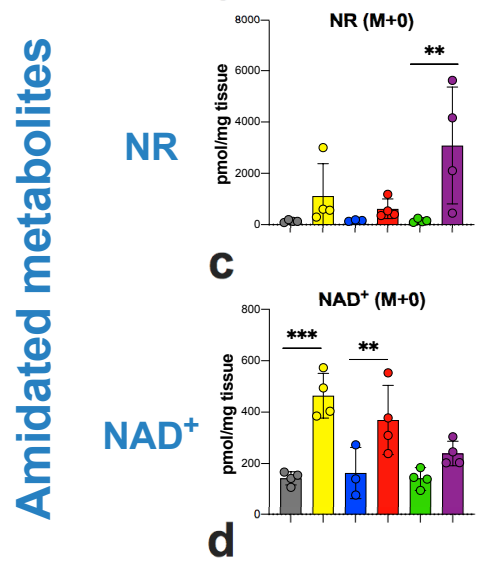

Nam

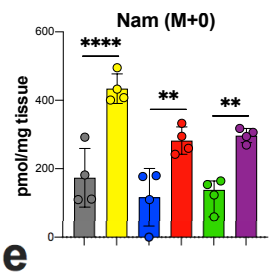

$\operatorname{Namn}(M+0)$
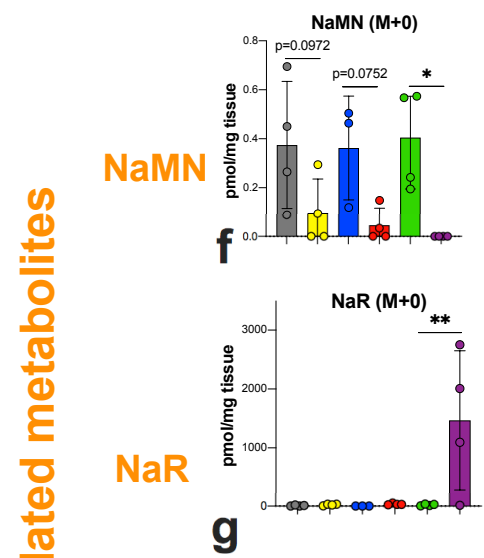

g

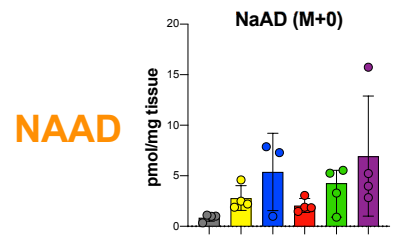

Antibiotics - + + - +

${ }^{15} \mathrm{~N}$-Glutamine $(\mathrm{M}+1)$

NMN1 (M+6)
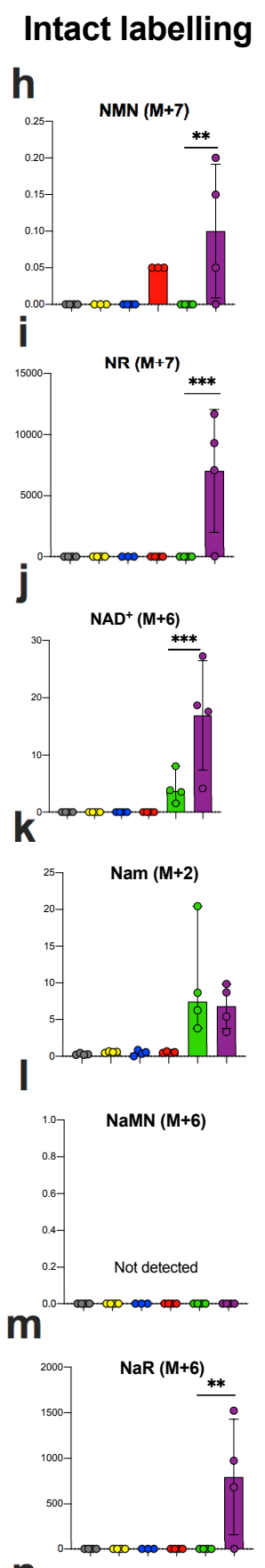

n

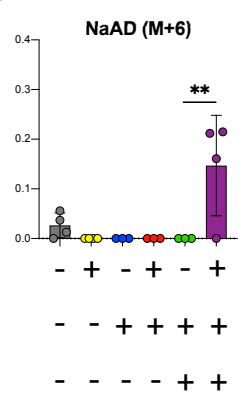

\section{Partial labelling}

o

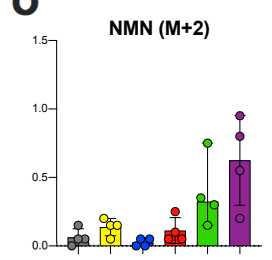

p

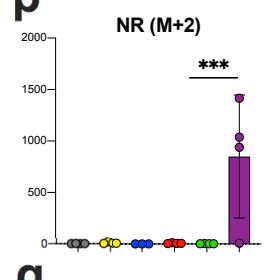

q
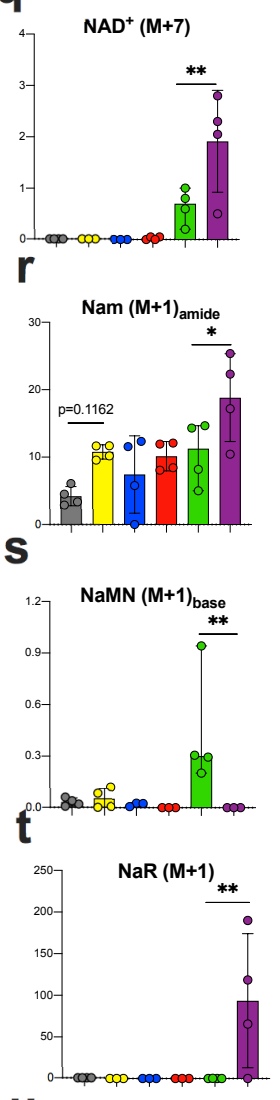

$\mathbf{u}$

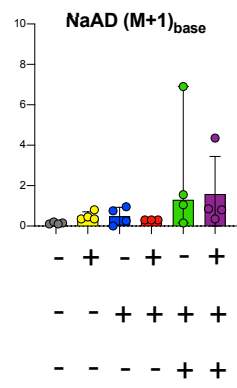

Supplementary Figure 4. Incorporation of NMN1 (M+6) into the GIT (raw data for Fig. 5). As in Figure 4 of main text, animals were treated with antibiotics (Abx) and $M+6$ isotope labelled 
NMN1 followed by metabolomics analysis of intestinal tissue as described in Figure 1 of main text.

Animals were also intraperitoneally injected with a concurrent bolus of ${ }^{15} \mathrm{~N}$-amide labelled glutamine $(735 \mathrm{mg} / \mathrm{kg}, 10 \mathrm{ml} / \mathrm{kg}$ body weight). The left column (a-g) represents the abundance of unlabelled, endogenous species, middle column (h-n) represents intact label incorporation, right column (o-u) represents partial labelled species from the recycling of metabolites, as predicted in Fig. 2a. Data analysed by 2-way ANOVA with Sidak's post-hoc test. n=3-4 animals per group, each data point represents a separate animal, error bars are SD. 


\section{Supplementary Figure 5}

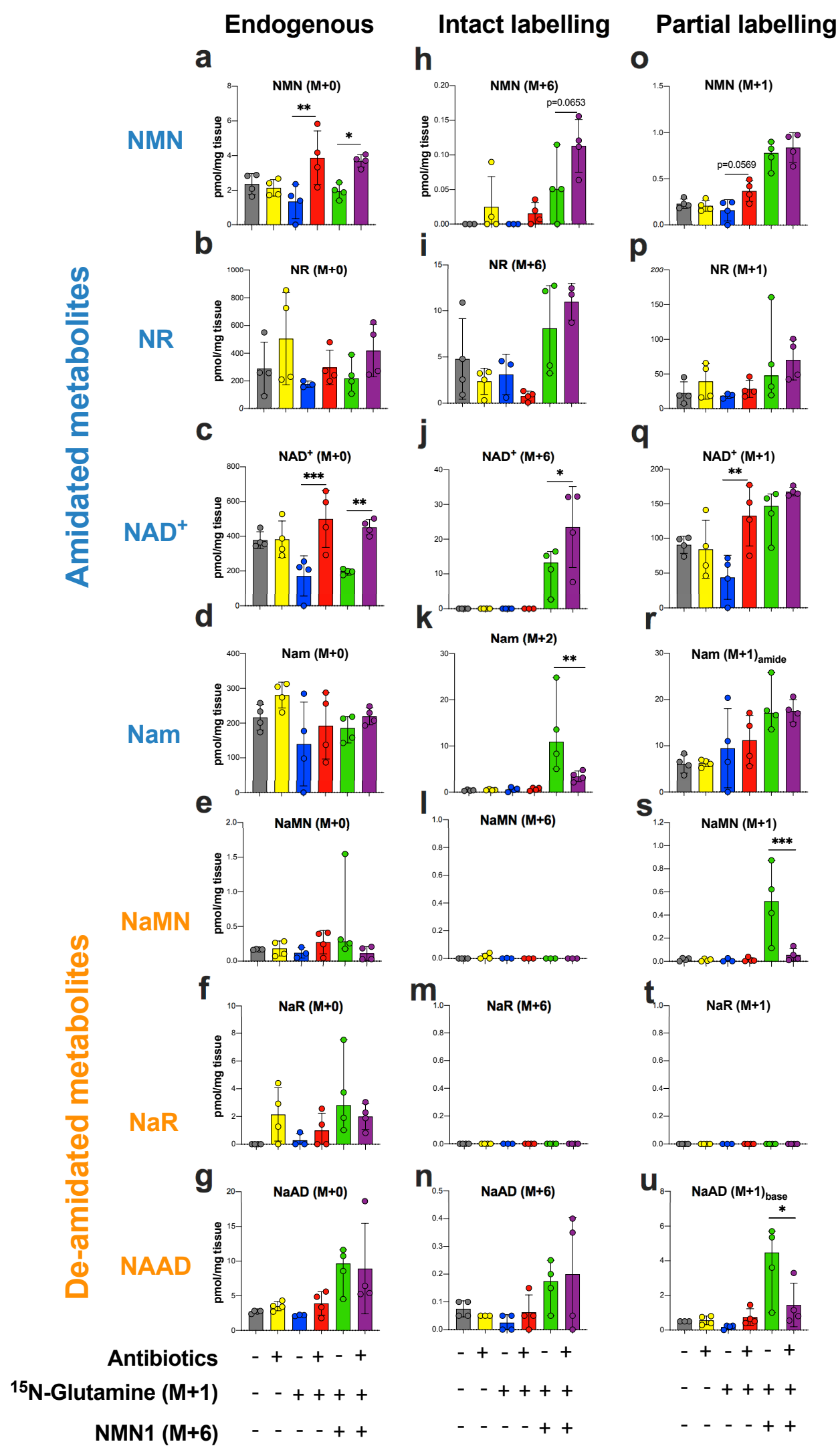

Supplementary Figure 5. Incorporation of NMN1 (M+6) into the liver (raw data for Fig. 5).

As in Figure 4 of main text, animals were treated with antibiotics (Abx) and M+6 isotope labelled

NMN1 followed by metabolomics analysis of intestinal tissue as described in Figure 1 of main text. 
Animals were also intraperitoneally injected with a concurrent bolus of ${ }^{15} \mathrm{~N}$-amide labelled glutamine $(735 \mathrm{mg} / \mathrm{kg}, 10 \mathrm{ml} / \mathrm{kg}$ body weight). The left column (a-g) represents the abundance of unlabelled, endogenous species, middle column (h-n) represents intact label incorporation, right column (o-u) represents partial labelled species from the recycling of metabolites, as predicted in Fig. 2a. Data analysed by 2-way ANOVA with Sidak's post-hoc test. $n=3-4$ animals per group, each data point represents a separate animal, error bars are SD. 


\section{Supplementary Figure 6}

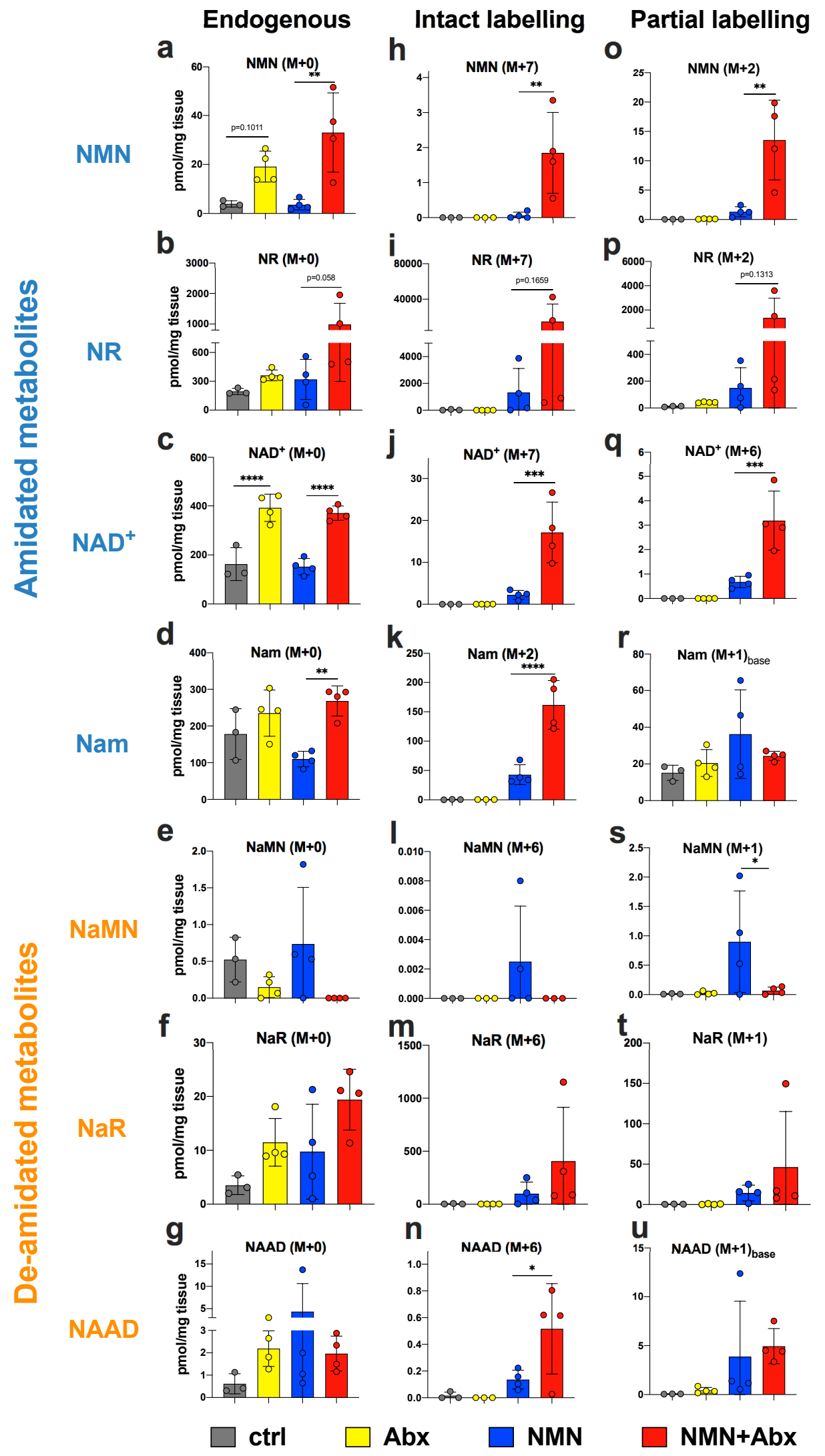

Supplementary Figure 6. Incorporation of NMN2 (M+7) into the GIT (raw data for Fig. 6).

Animals were treated with antibiotics $(\mathrm{Abx})$ and $\mathrm{M}+7$ isotope labelled NMN2 followed by metabolomics analysis of GIT tissue as described in Figure 6 of main text. Each data point represents 
measurements from a different animal. The left column (a-d) represents the abundance of unlabelled, endogenous species, middle column (e-h) represents intact label incorporation, right column (i-l) represents partial labelled species from the recycling of metabolites, as predicted in Fig. 1. Metabolites assayed are nicotinamide riboside (NR), nicotinamide adenine dinucleotide $\left(\mathrm{NAD}^{+}\right)$, nicotinamide (Nam) and nicotinamide mononucleotide (NMN). Data are analysed by two-way ANOVA with Sidak's multiple comparisons test. Data are mean \pm s.d. ( $n=3-5$ mice per group). ${ }^{*} \mathrm{p}<0.05, * * \mathrm{p}<0.01, * * * \mathrm{p}<0.001, * * * \mathrm{p}<0.0001, \mathrm{~ns}=$ not significant. 


\section{Supplementary Figure 7}

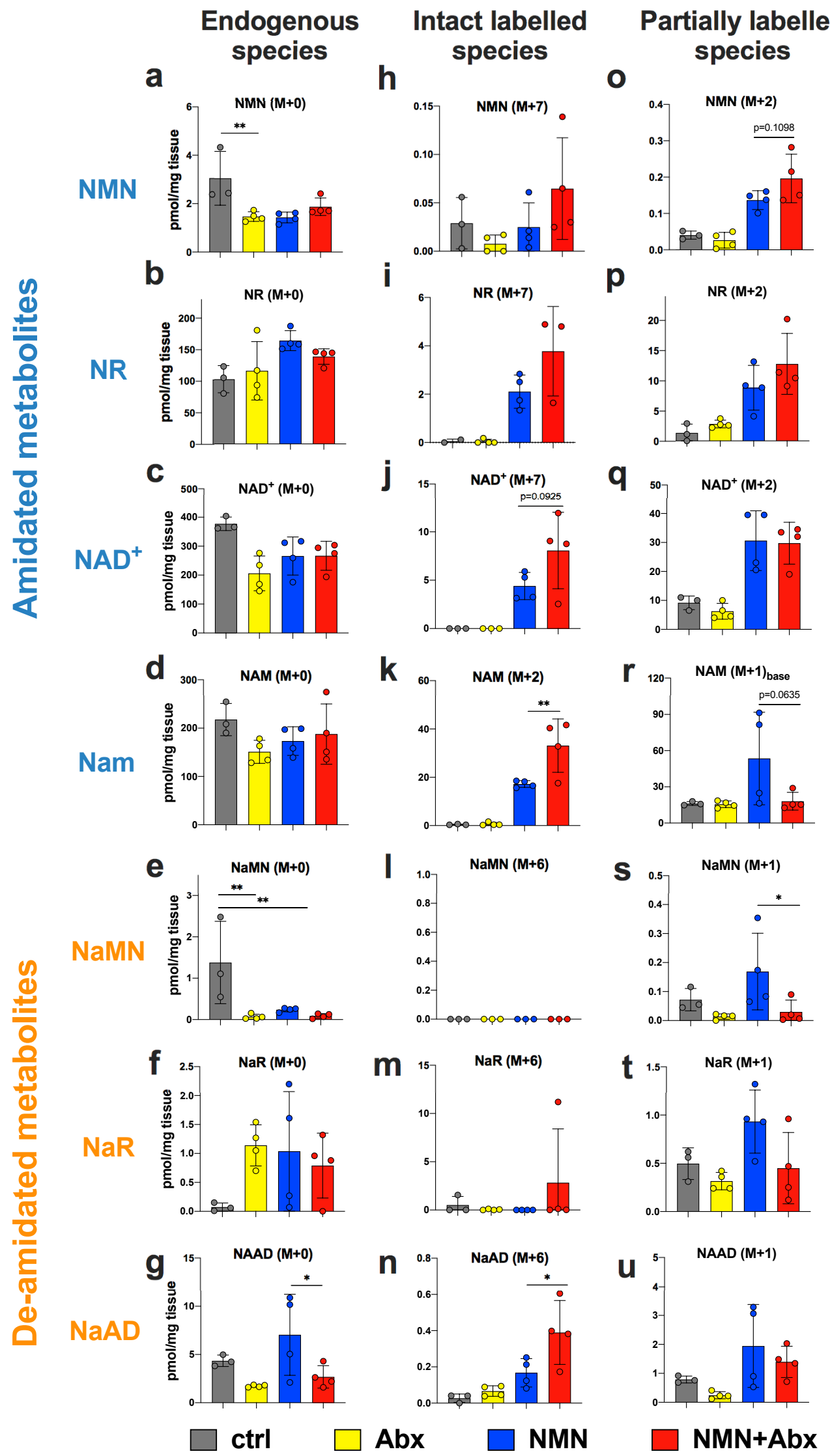

Supplementary Figure 7. Incorporation of NMN2 (M+7) into the liver (raw data for Fig. 6).

Animals were treated with antibiotics $(\mathrm{Abx})$ and $\mathrm{M}+7$ isotope labelled NMN2 followed by 
metabolomics analysis of liver tissue as described in Figure 6 of main text. Each data point represents measurements from a different animal. The left column (a-d) represents the abundance of unlabelled, endogenous species, middle column (e-h) represents intact label incorporation, right column (i-1) represents partial labelled species from the recycling of metabolites, as predicted in Fig. 1. Metabolites assayed are nicotinamide riboside (NR), nicotinamide adenine dinucleotide $\left(\mathrm{NAD}^{+}\right)$, nicotinamide (Nam) and nicotinamide mononucleotide (NMN). Data are analysed by two-way ANOVA with Sidak's multiple comparisons test. Data are mean \pm s.d. ( $n=3-5$ mice per group). ${ }^{*} \mathrm{p}<0.05, * * \mathrm{p}<0.01, * * * \mathrm{p}<0.001, * * * * \mathrm{p}<0.0001, \mathrm{~ns}=$ not significant. 


\section{Supplementary Figure 8}
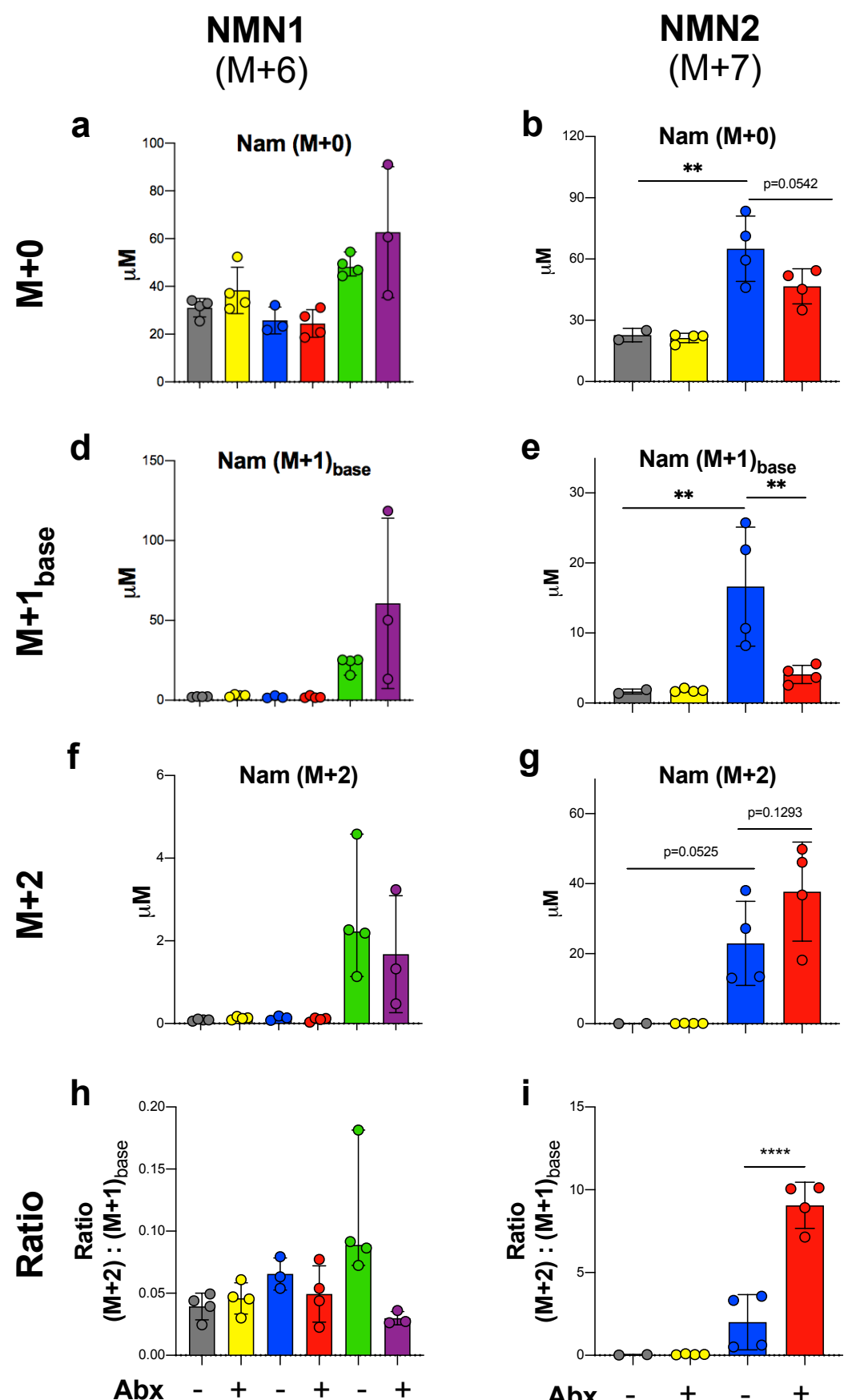

${ }^{15} \mathrm{~N}-\mathrm{G} \ln (\mathrm{M}+1)-\quad+\quad+\quad+$

NMN1 $(M+6)-\quad-\quad-\quad+$ e

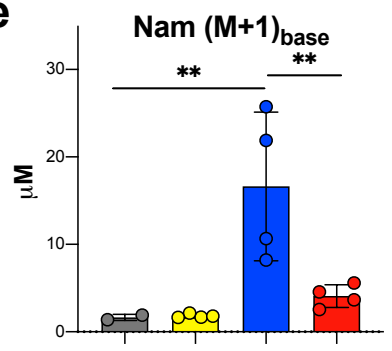

9

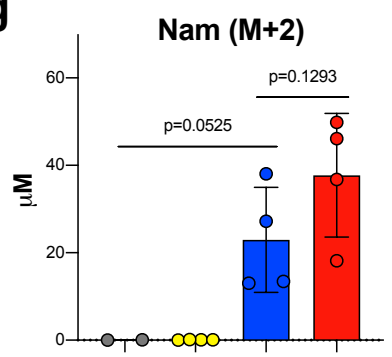

i

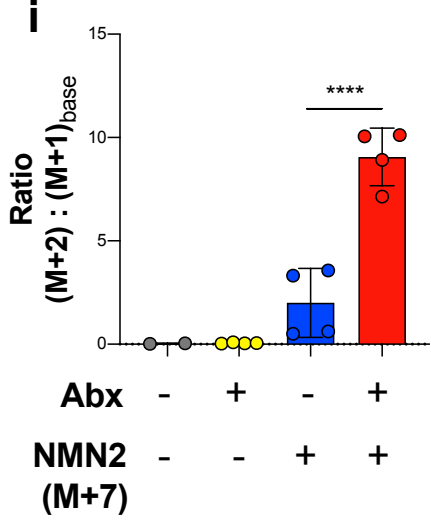

Unlabelled NMN

$(\mathrm{M}+0)$

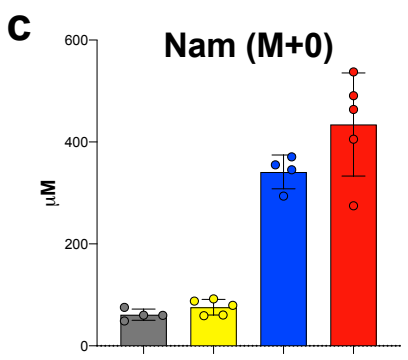

Abx - + - +

NMN - -++

Supplementary Figure 8. Plasma nicotinamide levels. (a-c) Unlabelled, (d, e) $\mathrm{M}+1_{\text {base }}$ (i.e. nicotinyl ring $\left.{ }^{15} \mathrm{~N}\right)$, (f, g) $\mathrm{M}+1_{\text {amide }}$ and $(\mathrm{h}, \mathrm{i}) \mathrm{M}+2$ labelled Nam in antibiotic (Abx) treated animals receiving (a, d, f, g) NMN1 $(\mathrm{M}+6),(\mathrm{b}, \mathrm{e}, \mathrm{g}, \mathrm{i}) \mathrm{NMN} 2(\mathrm{M}+7)$ and $(\mathrm{c})$ unlabelled nicotinamide, as described in main Fig. 1, 5, and 6. Data are analysed by two-way ANOVA with Sidak's multiple comparisons test. 


\section{Supplementary Figure 9}

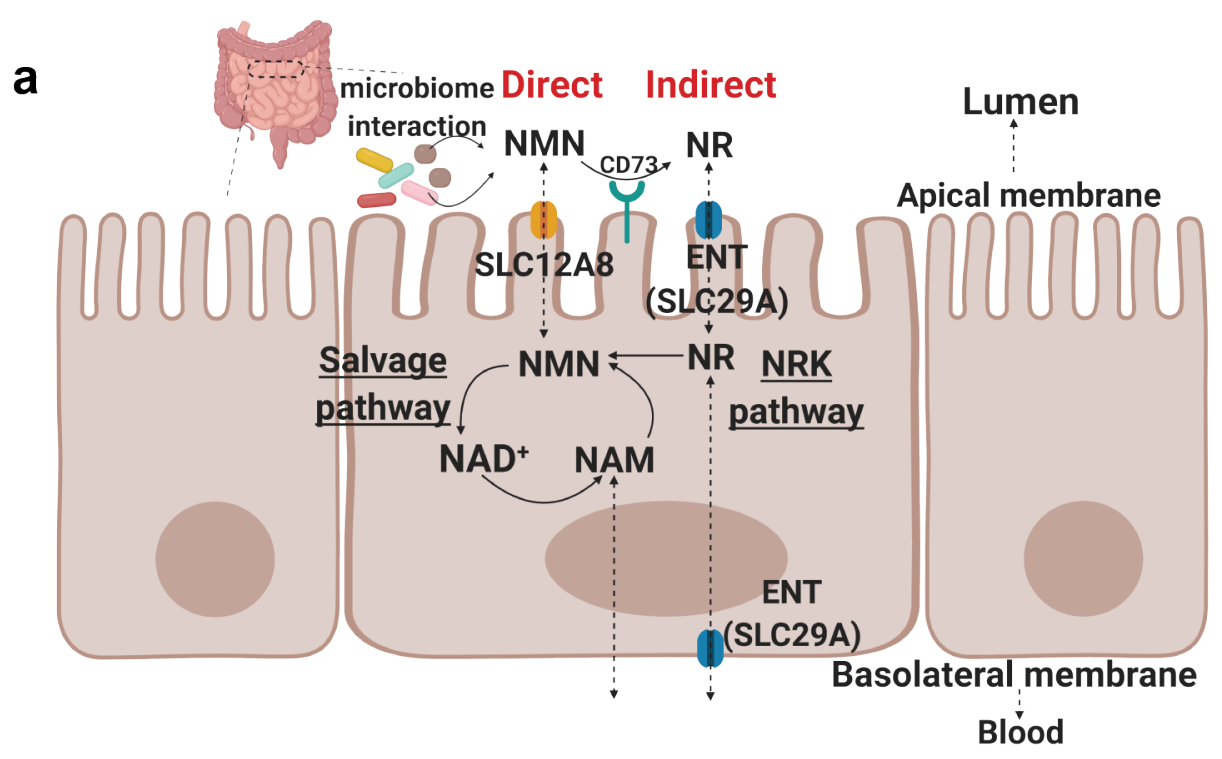

b

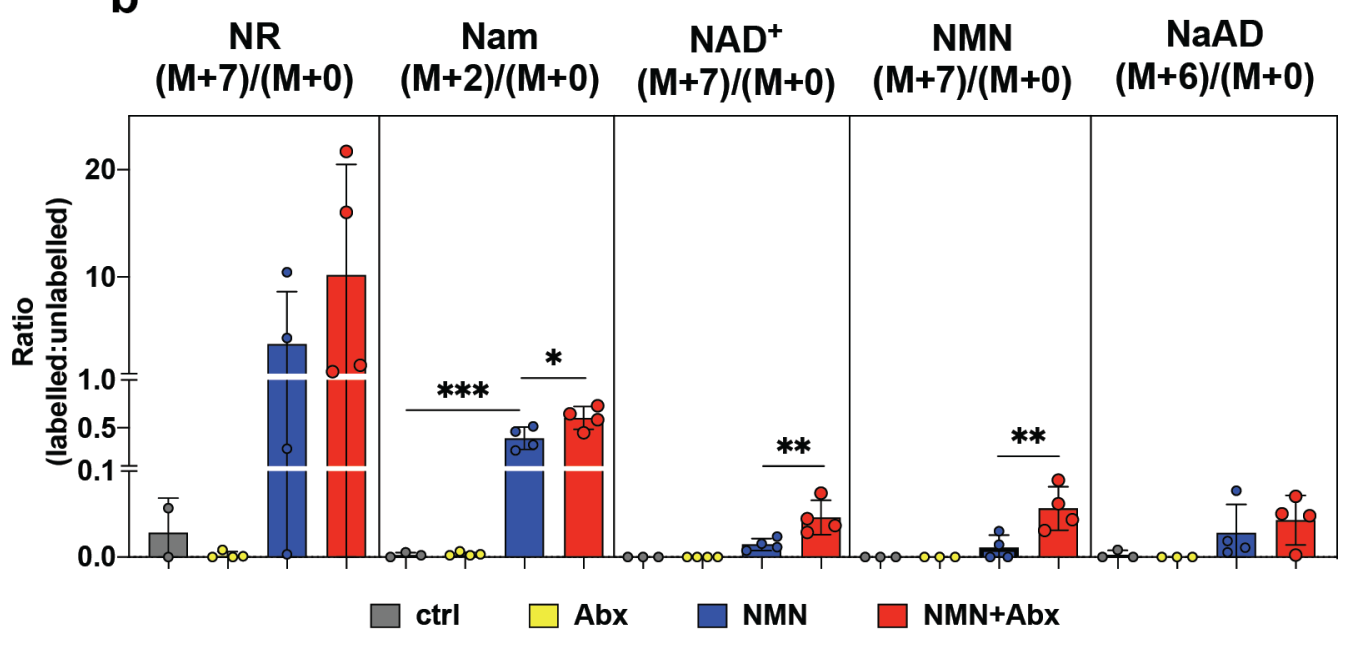

Supplementary Figure 9. Isotope labelled NMN treatment results in greater labelling of the NR

than NMN pool, suggesting indirect uptake. (a) The two proposed mechanisms for NMN uptake are either directly through the putative NMN transporter SLC12A8, or indirectly by dephosphorylation into NR via the ecto-5'-nucleotidase CD73 which is present on the apical side of intestinal cells. To compare the contributions of either direct or indirect transport mechanisms, the contribution of isotope labelled NMN to the overall pool of each metabolite is shown for the intestinal tissue of the NMN2 cohort (data from Fig. 6). Error bars are s.d., each data point represents tissue from a separate animal. 


\section{Supplementary Figure 10}
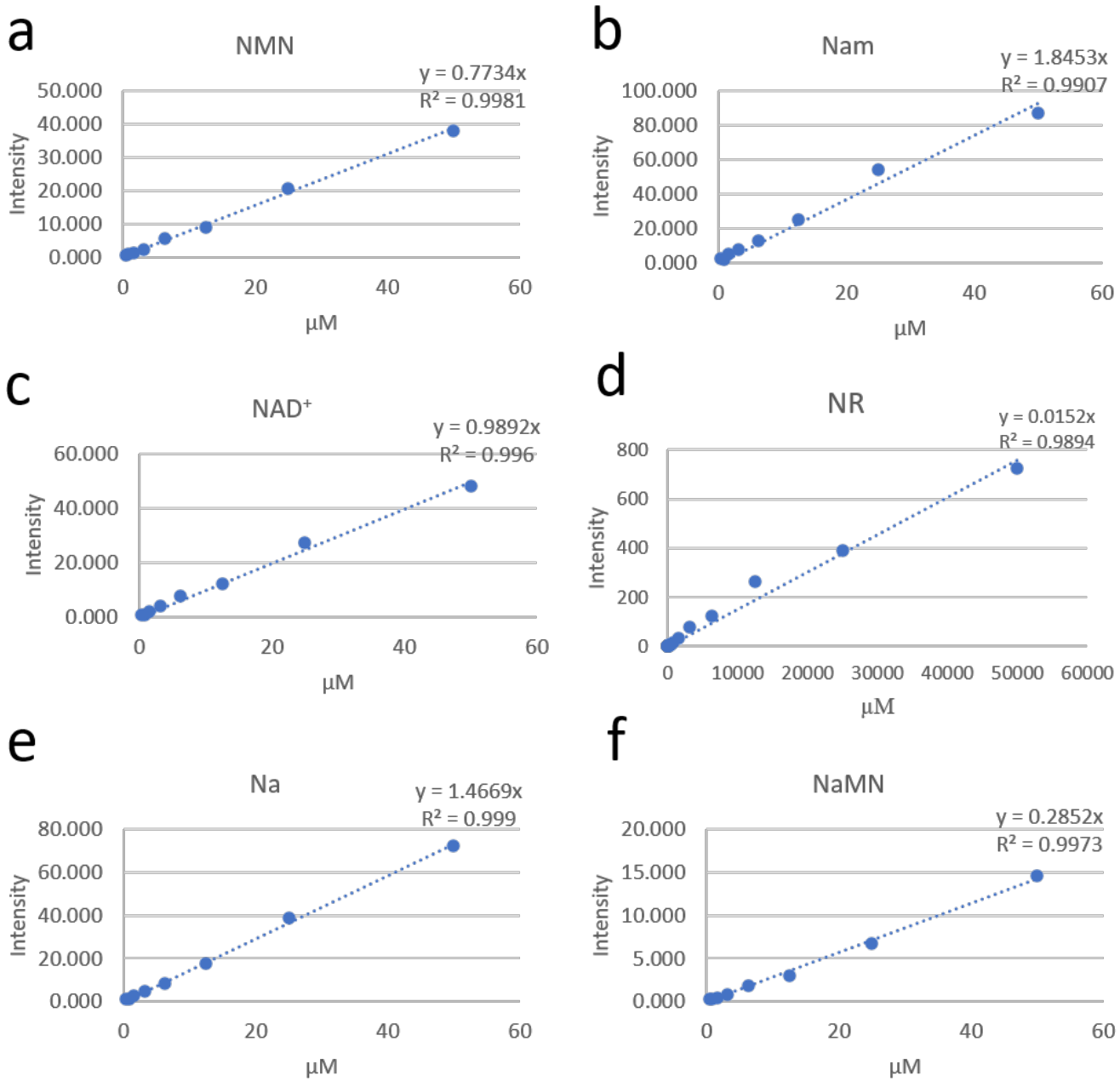

$f$
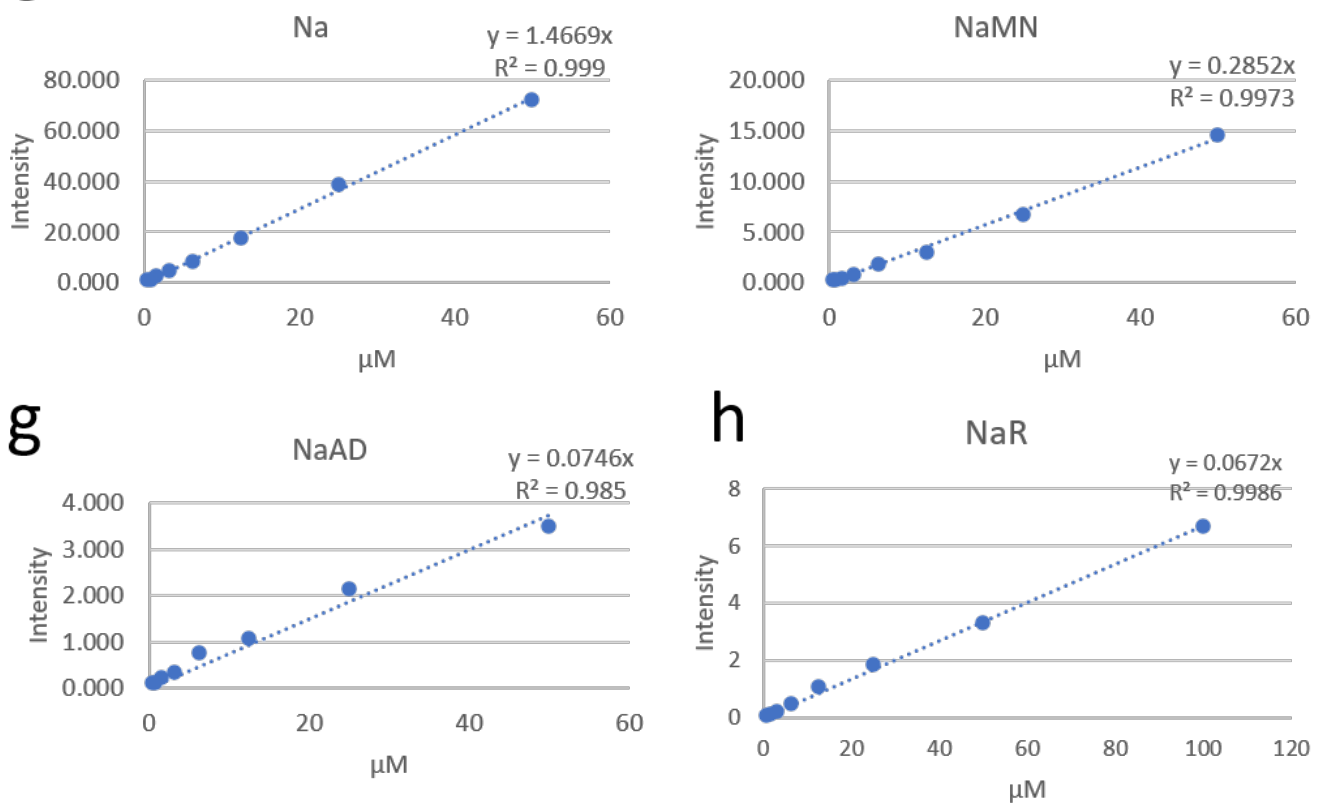

Supplementary Figure 10. NAD ${ }^{+}$metabolite standard curves. (a) NMN (b) Nam (c) $\mathrm{NAD}^{+}$(d)

NR (e) $\mathrm{Na}$ (f) $\mathrm{NaMN}$ (g) NaAD (h) NaR. Standard curves were serially diluted from $50 \mu \mathrm{M}$ to 0.39 $\mu \mathrm{M}$. Shown here from $50 \mu \mathrm{M}$ to $0.39 \mu \mathrm{M}$ for all metabolites except for NR which is shown from 50 $\mathrm{mM}$ to $0.39 \mu \mathrm{M}$, due to the unexpectedly high concentrations of NR in the GIT (Fig. 1, 5, 6) and NaR from $100 \mu \mathrm{M}$ to $0.39 \mu \mathrm{M}$. 


\section{Supplementary Figure 11}
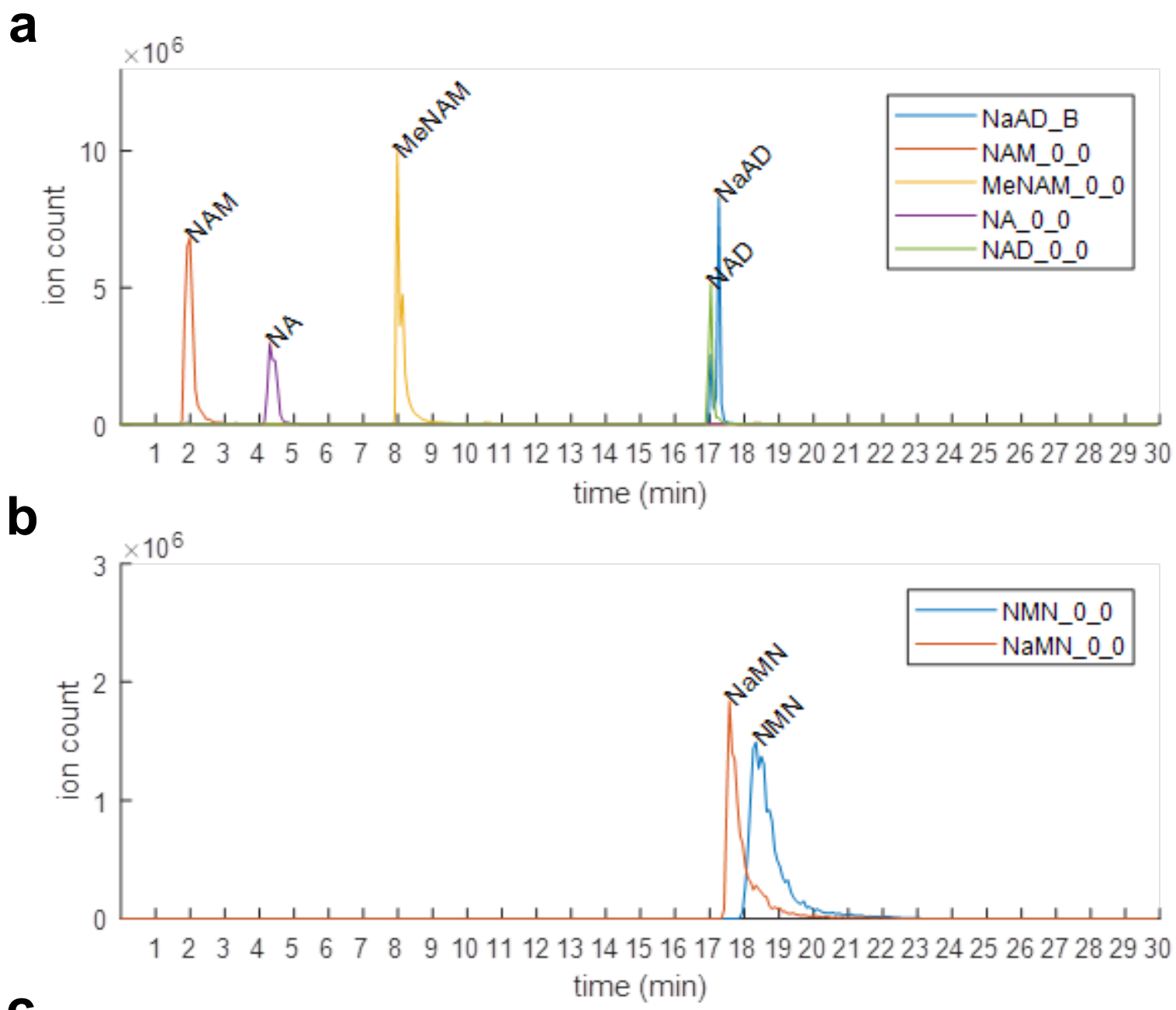

C

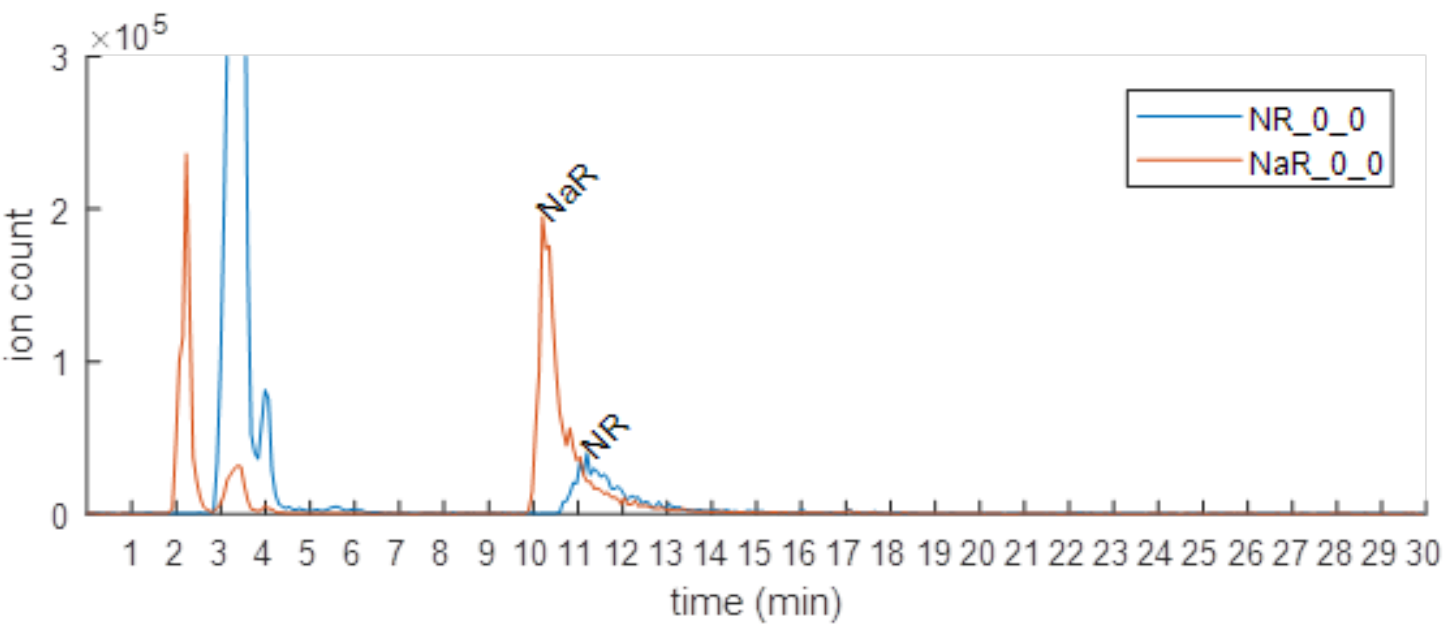

Supplementary Figure 11. Chromatograms of NAD ${ }^{+}$metabolites using MRM LC-MS/MS. Above chromatograms represent individual peaks (ion count) from $100 \mu \mathrm{M}$ standard solutions at each respective retention times for (a) NAM, NA, MeNAM, NAD ${ }^{+}$and NAAD, (b) NAMN and NMN, (c) NAR and NR. 


\section{SUPPLEMENTARY METHODS}

\section{Synthesis of isotope labelled NMN}

The isotopes used here were generated through a two-step process starting with the custom synthesis of nicotinamide labelled with ${ }^{15} \mathrm{~N}$ at the nitrogen base and amide positions. This custom isotope labelled version of nicotinamide was then used with [U5]- ${ }^{13} \mathrm{C}$ - ribose which was ${ }^{13} \mathrm{C}$ labelled at all five carbon positions (Cambridge Isotope Laboratories, cat. no. CLM-3652) and ATP in an enzymebased protocol using recombinant phosphoribosyl synthetase (PRS) and recombinant nicotinamide phosphoribosyl transferase (NAMPT) to synthesise NMN. The two enzymes were added into the reaction buffer that contains $1 \mathrm{mM}$ ribose, $1 \mathrm{mM}$ nicotinamide, $3 \mathrm{mM}$ ATP, $1 \mathrm{mM}$ dithiothreitol, 10 $\mathrm{mM} \mathrm{MgCl} 2$ and $50 \mathrm{mM}$ Tris- $\mathrm{HCl}\left(\mathrm{pH} \mathrm{7.5)}\right.$ and incubated at $37^{\circ} \mathrm{C}$ for $30 \mathrm{~min}$. The reaction was terminated with the addition of $0.01 \%$ Trichloroacetic acid (TCA). The purification was proceeded with size-exclusion columns and ion exchange columns. Isotope labelled NMN samples of $>95 \%$ purity were concentrated by lyophilization, and labelling confirmed by mass spectrometry (Supp. Fig. 2).

\section{$\underline{\text { Animal experiments }}$}

All experiments were performed according to procedures approved by UNSW Animal Care and Ethics Committee (ACEC) under ethics protocol 18/134A. The UNSW ACEC operates under the animal ethics guidelines from the National health and Medical Research Council (NHMRC) of Australia. Mice were fed standard chow ad libitum and housed under a 12-hr light/12-hr dark cycle in a temperature-controlled room $\left(22 \pm 1^{\circ} \mathrm{C}\right)$ at $80 \%$ humidity in individually ventilated cages. Fourweek old female C57BL/6J mice were acclimatised for one week prior to treatment and body weight matched before random assignment into groups. For antibiotic treatment, mice were administered a cocktail of antibiotics consisting of vancomycin (0.5 g/L; Sigma SBR00001), neomycin (1 g/L; Sigma N6386), ampicillin (1 g/L; Sigma A9393) and metronidazole (1 g/L; Sigma, M3761) (VNAM) 
with addition of sucrose ( $3 \mathrm{~g} / \mathrm{L}$; Bundaberg Sugar) to increase palatability for 4 days, and switched to ampicillin ( $1 \mathrm{~g} / \mathrm{L})$ with sucrose $(3 \mathrm{~g} / \mathrm{L})$ for an additional week, which can reduce gut bacterial density by 1000-fold (Ubeda et al., 2010). During this treatment period there was a reduction in water consumption in the VNAM group (below), which was the reason for the switch to ampicillin alone.
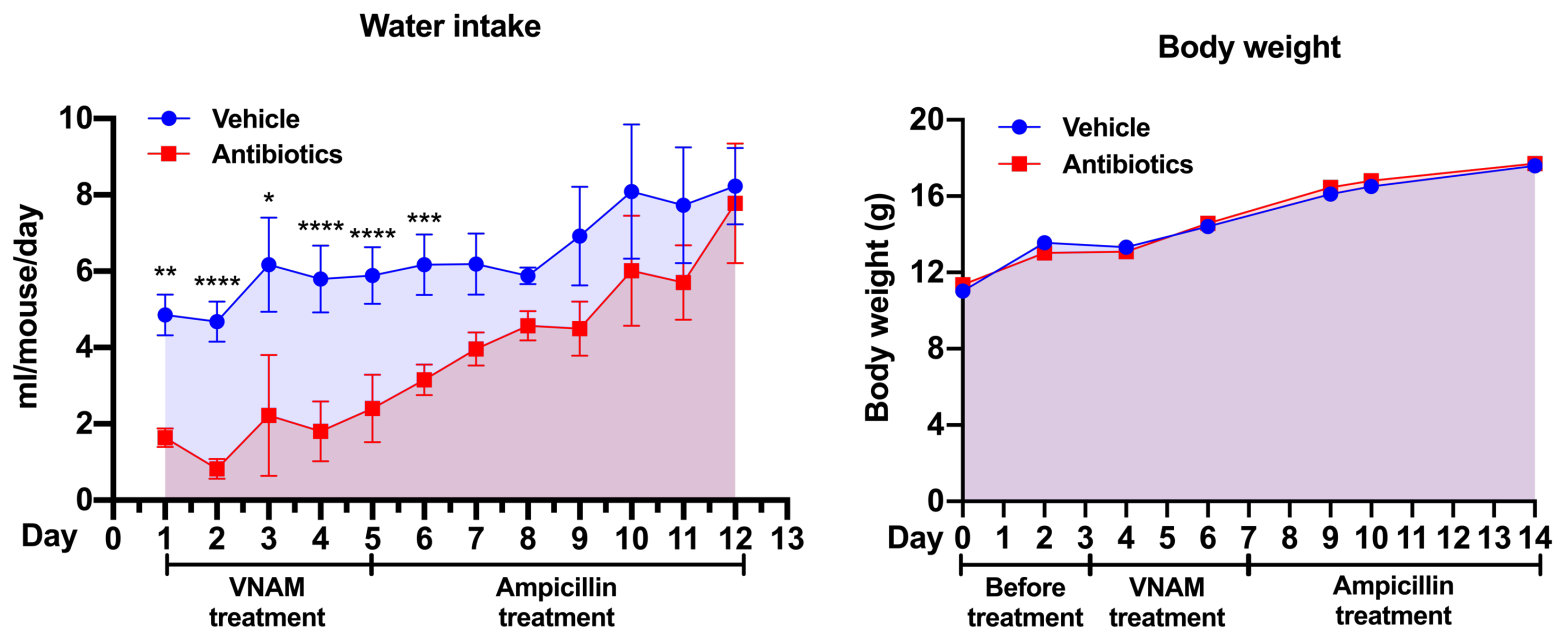

To maintain consistency, this antibiotic treatment protocol was used for all in vivo experiments (Figs. 1, 5, 6). For NMN treatment, mice received a single oral gavage of NMN1 (Fig. 5) or NMN2 (Fig. 6) isotopes at $50 \mathrm{mg} / \mathrm{kg}$, or for unlabelled NMN (Fig. 1) at $500 \mathrm{mg} / \mathrm{kg}$, with water vehicle used as a control. Four hours later, animals were placed under anaesthesia, and blood was obtained by cardiac puncture, followed by euthanasia by cervical dislocation, and rapid dissection and snap freezing of tissues. Gavages were staggered by 5 mins between mice with alternating treatment groups to avoid any experimental bias. On the day of cull mice were all 5-6 weeks old. Differences in NMN dosing between unlabelled and isotope labelled NMN were due to limited availability of isotope labelled material.

\section{Blood plasma collection and preparation for mass spectrometry}

Approximately $1 \mathrm{~mL}$ of blood was collected via cardiac puncture in anaesthetised mice (1-2\% isoflurane) into $1.5 \mathrm{~mL}$ eppendorf tubes prefilled with $10 \mu \mathrm{L}$ of EDTA $(0.5 \mathrm{M})$ and mixed thoroughly 
with a pipette to prevent clotting. Blood samples were then spun at $2000 \mathrm{~g}$ for $10 \mathrm{~min}$ and the top layer was transferred to a new tube and snap frozen immediately in liquid nitrogen. All samples were stored in $-80^{\circ} \mathrm{C}$ until further processing. On the day of sample acquisition plasma samples were thawed on ice and $20 \mu \mathrm{L}$ of plasma was added to $80 \mu \mathrm{L}$ of extraction buffer (acetonitrile:methanol) with an internal standard mixture containing MES, CSA and thymine-d4. Samples were vortexed and centrifuged at $16,000 \mathrm{~g}$ for $10 \mathrm{mins}$ at $4^{\circ} \mathrm{C}$ and the supernatant was transferred to a new eppendorf tube and dried down completely using a speed vacuum concentrator (Savant SpeedVac ${ }^{\circledR}$ SPD140DDA, Thermo Scientific). The resulting pellet was then resuspended in $30 \mu \mathrm{L}$ of LC-MSgrade water and centrifuged as above and the supernatant analysed promptly by LC-MS.

\section{$\underline{\text { Gastrointestinal and liver tissue collection and preparation for mass spectrometry }}$}

Intestinal contents (small intestine and colon without cecum) were resected and flushed with ice cold $1 \times$ phosphate buffered saline (PBS) to clear faecal contents before snap freezing immediately with liquid nitrogen. Livers were resected and weighed before being rinsed in ice cold 1x PBS and snap frozen in liquid nitrogen. All tissue samples were stored in $-80{ }^{\circ} \mathrm{C}$ until further processing. Frozen tissue samples were crushed using a mortar and pestle on liquid nitrogen and approximately $50 \mathrm{mg}$ was weighed into tubes containing ceramic beads (Precellys, Bertin Technologies, France) to which $500 \mu$ of cold $\left(-30^{\circ} \mathrm{C}\right)$ extraction buffer (acetonitrile:methanol:water, 2:2:1) with internal standard mixture as above was added. All samples were homogenised using an automated tissue homogeniser (Precellys24, Bertin Technologies, France) at 5,000-6,000 rpm for 15 seconds and immediately centrifuged at $16,000 \mathrm{~g}$ for $10 \mathrm{mins}$ at $4{ }^{\circ} \mathrm{C}$. The supernatant was transferred to a new tube and dried down completely using a speed vacuum concentrator (Savant SpeedVac ${ }^{\circledR}$ SPD140DDA, Thermo Scientific). All samples were resuspended in $50 \mu \mathrm{L}$ of LC-MS-grade water, centrifuged as above and the supernatant analysed promptly by LC-MS. 


\section{Bacterial culture and NMN treatment}

A stab culture of the $E$. coli strain OP50 was inoculated into sterile Luria-Bertani (LB) broth (10 g/L tryptone, $5 \mathrm{~g} / \mathrm{L}$ yeast and $10 \mathrm{~g} / \mathrm{L}$ sodium chloride in deionized water) under aseptic conditions and incubated overnight at $37^{\circ} \mathrm{C}$ on a shaking platform set at $200 \mathrm{rpm}$. To measure the growth rate of $E$. coli, the overnight culture was sub-cultured (1:200) into sterile LB broth in a new flask and the optical density was measured at $600 \mathrm{~nm}\left(\mathrm{OD}_{600}\right)$ every 20 minutes (approximate doubling time) and samples were collected during the early-mid exponential growth phase (OD600 $<0.70)$, as bacterial enzymes are more active during exponential growth phase than stationary phase (Rahman et al., 2006). For samples, the overnight culture was sub-cultured (1:200) and aliquoted into smaller volumes. The cultures were then supplemented with either vehicle (water) or M+6 labelled NMN (0.1 mM) and OD600 measured at time zero (before NMN), time zero (after NMN), and 140, 160 and 180 minutes after supplementation with NMN. The supernatant of cells was separated from the cells via centrifugation $\left(5000 \mathrm{~g}\right.$ for 10 minutes at $\left.4^{\circ} \mathrm{C}\right)$ and stored immediately at $-30^{\circ} \mathrm{C}$. Meanwhile, the cell pellet was resuspended in cold $\left(4^{\circ} \mathrm{C}\right)$ saline solution $(0.9 \% \mathrm{NaCl})$ and centrifuged as above, to rinse away residual media before storage at $-30^{\circ} \mathrm{C}$. The $\mathrm{OD}_{600}$ was measured for each sample and used to normalise metabolite levels after LC-MS/MS analysis.

\section{Primary hepatocyte culture}

Primary hepatocytes were obtained as described previously (Chowdhury et al., 2016). Male Sprague Dawley rats (250 grams, Animal Resources Centre, Perth, WA, Australia) were maintained on a 12:12 h day-night cycle, with water and food supplied ad libitum. Under deep non-recoverable general anaesthesia (75 mg/kg ketamine, $10 \mathrm{mg} / \mathrm{kg}$ xylazine, intraperitoneal administration) rats underwent laparotomy. The portal vein was cannulated in situ and the liver perfused initially with carbogensaturated perfusion media (final: $\mathrm{NaCl} 138 \mathrm{mM}$, HEPES $25 \mathrm{mM}$, D-glucose $5.6 \mathrm{mM}, \mathrm{KCl} 5.4 \mathrm{mM}$, $\mathrm{Na}_{2} \mathrm{HPO}_{4}, 0.34 \mathrm{mM}, \mathrm{KH}_{2} \mathrm{PO}_{4} 0.44 \mathrm{mM}, \mathrm{NaHCO}_{3} 4.17 \mathrm{mM}$, EDTA $0.5 \mathrm{mM}, \mathrm{pH} 7.4,37^{\circ} \mathrm{C}, 25 \mathrm{ml} / \mathrm{min}$ flow rate). The inferior vena cava was cut to allow efflux. After 4 mins, the carbogen-saturated 
perfusion media was changed to the collagenase containing buffer (final: $\mathrm{NaCl} 138 \mathrm{mM}$, HEPES 25 mM, D-glucose $5.6 \mathrm{mM}, \mathrm{KCl} 5.4 \mathrm{mM}, \mathrm{Na}_{2} \mathrm{HPO}_{4}, 0.34 \mathrm{mM}, \mathrm{KH}_{2} \mathrm{PO}_{4} 0.44 \mathrm{mM}, \mathrm{NaHCO}_{3} 4.17 \mathrm{mM}$,

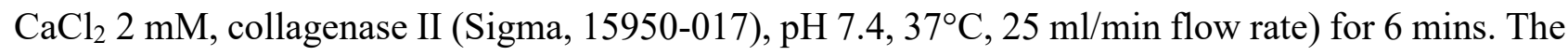
inferior vena cava was clamped at least 10 times during the collagenase digestion (preventing efflux) resulting in liver swelling that allows a better digestion.

Following the collagenase digestion, the liver was removed and place on ice in $20 \mathrm{ml}$ Williams' Medium E (Life-technologies, Waltham, MA, USA). The hepatocytes were gently dispersed in the medium and the cells filtered through a $100 \mu \mathrm{m}$ cell strainer. Hepatocytes were washed and diluted Williams' Medium E and plated (6-well plates) at 106/2ml/well. After $4 \mathrm{hrs}$ of incubation the culture medium was changed to MOPS buffer (final, $\mathrm{NaCl} 128 \mathrm{mM}$, MOPS $23.9 \mathrm{mM}, \mathrm{KCl} 6 \mathrm{mM}$, $\mathrm{MgSO}_{4} .7 \mathrm{H}_{2} \mathrm{O} 1.18 \mathrm{mM}, \mathrm{CalCl}_{2} 1.29 \mathrm{mM}$, glucose $5 \mathrm{mM}$, BSA (FFA) $0.2 \%$, pH 7.4) and cells incubated overnight. Following overnight incubation, cells were incubated in M199 media without glutamine (Sigma M2154), supplemented with either unlabelled (Sigma) or ${ }^{15} \mathrm{~N}$-amide labelled glutamine (Cambridge Isotope Laboratories NLM-557) at $4 \mathrm{mM}$, in the presence or absence of NMN1 or NMN2 isotopes at $200 \mu \mathrm{M}$ for $24 \mathrm{hr}$, following which samples were preserved for metabolomic analysis (Fig. 3).

\section{$\underline{\text { Preparation of } \mathrm{NAD}^{+} \text {metabolite standards }}$}

$\mathrm{NAD}^{+}$metabolites were serially diluted starting from a concentration of $100 \mu \mathrm{M}$ to $0.39 \mu \mathrm{M}$. The same volume $(500 \mu \mathrm{L})$ of extraction buffer (acetonitrile:methanol:water) was added and vortexed before centrifuging and transferring to new tube ready to be dried down as above. The subsequent steps were the same as preparing the tissue samples as above. All standards and samples were processed on the same day to reduce any experimental bias or variability. Standard curves used to calculate absolute concentrations are shown in Supp. Fig. 10 and are available in supplementary raw data files. 


\section{Mass spectrometry}

The LC-MS method was performed using 1260 Infinity LC System (Agilent) coupled to QTRAP 5500 (AB Sciex) mass spectrometer. LC separation by gradient elution was accomplished on an XBridge BEH amide column (100 mm x $2.1 \mathrm{~mm}, 3.5 \mu \mathrm{m}$ particle size, Waters Corporation) at room temperature. For the mobile phase, Solvent A is 95\%:5\% $\mathrm{H}_{2} \mathrm{O}$ :acetonitrile containing $20 \mathrm{mM}$ ammonium acetate and $20 \mathrm{mM}$ acetic acid, and solvent $\mathrm{B}$ is acetonitrile. The flow rate was 200 $\mu \mathrm{L} / \mathrm{min}$, with the percentage of solvent B set at $85 \%(0 \mathrm{~min}), 85 \%(0.1 \mathrm{~min}), 70 \%(10 \mathrm{~min}), 15 \%(13$ min), 15\% (17 $\mathrm{min}), 85 \%$ (17.5 $\mathrm{min}), 85 \%$ (30 $\mathrm{min}$ ) (Supplementary Table 1). Injection volume was $2.5 \mu \mathrm{L}$. Ion source was set at $350{ }^{\circ} \mathrm{C}$ and $4500 \mathrm{~V}$ with polarity switching. Mass isotopologues of metabolites were acquired by $\mathrm{MS}^{2}$, using the unscheduled multiple reaction monitoring (MRM) mode with a dwell time of $40 \mathrm{~ms}$. The MS parameters (declustering potential, collision energy and cell exit potential) (Supplementary Table 2a) and MRM transitions were calibrated based on the monoisotopic mass of chemical standards (Supplementary Figure 11). Data processing was performed using MSConvert (version 3.0.18165-fd93202f5) and in-house MATLAB scripts. Deconvolution scripts were developed to resolve overlapping NAAD-NAD, NAR-NR and NAMN-NMN peaks using MATLAB's Optimisation Toolbox. Representative chromatograms are shown in Supp. Fig. 11.

\section{$\underline{\text { Statistical analysis for mass spectrometry }}$}

All data are presented as mean \pm standard deviation (s.d.). Statistical significance was performed using a two-way ANOVA with a Sidak's multiple comparisons test to determine differences between groups after removing outliers using the ROUT method $(\mathrm{Q}=1 \%)$. Data in Fig. 7 were analysed by Mann-Whitney U-test between NMN isotope treated groups due to the absence of detection of labelled metabolites in animals that did not receive NMN isotopes. All statistics were performed on GraphPad Prism software (version 8.2.1). P values less than 0.05 were considered statistically significant. All data analyses are available as an .xml file available on our Mendeley data site. For in vivo experiments (Fig. 1, 5-8, Supp. Fig. 4-7), each data point represents tissues from a separate 
animal, while each data point for in vitro experiments (Fig. 2,3) represents an independent biological replicate.

\section{DNA extraction from faecal pellets}

Solid faecal pellets taken from the colonic and rectal region of the gastrointestinal tract were stored in $-80^{\circ} \mathrm{C}$ until further processing. DNA was extracted from frozen faecal pellets using the QIAamp ${ }^{\circledR}$ PowerFecal ${ }^{\circledR}$ DNA kit (Qiagen, Cat. No. 12830-50) according to the manufacturer's protocol. DNA concentration was determined using the $\operatorname{NanoDrop}^{\mathrm{TM}}\left(\right.$ DeNovix ${ }^{\circledR}, \mathrm{DS}-11$ FX) and the purity of double-stranded DNA (dsDNA) was also determined by measuring the 260/280 ratio. All DNA extracts were stored at $-80^{\circ} \mathrm{C}$ until further processing by $16 \mathrm{~S}$ rRNA sequencing.

\section{Nanopore 16S Sequencing}

Full length 16S rRNA genes were amplified by PCR using the Oxford Nanopore 16S Barcoding Kit (SQK-RAB204; Oxford Nanopore Technologies, Oxford, UK). Briefly, 10 ng genomic DNA, $1 \mu \mathrm{L}$ 16S Barcode $(10 \mu \mathrm{M})$ and $25 \mu \mathrm{L}$ LongAmp Taq 2X Master Mix (New England Biolabs, Ipswich, MA, USA) were combined in a $50 \mu \mathrm{L}$ reaction for PCR on a Bio-Rad T100 ${ }^{\mathrm{TM}}$ Thermal Cycler (BioRad Laboratories Pty Ltd, Hercules, CA, USA). PCR cycling condition were as follows; initial denaturation at $95{ }^{\circ} \mathrm{C}$ for 1 minute, 25 cycles of denaturation at $95{ }^{\circ} \mathrm{C}$ for 20 seconds, annealing at 55 ${ }^{\circ} \mathrm{C}$ for 30 seconds and extension at $65^{\circ} \mathrm{C}$ for 2 minutes before a final extension at $65^{\circ} \mathrm{C}$ for 5 minutes. PCR products were purified as per Oxford Nanopore Technologies (ONT) protocol using AMPure XP magnetic beads (Beckman Coulter, Indianapolis, IN) and DNA quantified using the NanoDrop ${ }^{\mathrm{TM}}$ (DeNovix ${ }^{\circledR}$, DS-11 FX). Barcodes were pooled to a total of $100 \mathrm{fmol}$ in $10 \mu \mathrm{L}$ of $10 \mathrm{mM}$ Tris-HCl, pH 8.0 with $50 \mathrm{mM} \mathrm{NaCl}$ for library loading. Sequencing was performed using R9.4.1 ONT Flow Cells on the MinION ${ }^{\mathrm{TM}}$ sequencing platform and data acquired using MinKNOW software version 19.10.1 (Oxford Nanopore Technologies). 


\section{Data Analysis for $16 \mathrm{~S}$ sequencing}

Full length 16S sequencing reads acquired from MinION runs (i.e. FAST5 data) were base-called to fastq files using Guppy software version 3.4.4 (Oxford Nanopore Technologies). Fastq files were demultiplexed using Porechop (https://github.com/rrwick/Porechop) and trimmed to 1400bp with Trimmomatic version 0.39 (Bolger et al., 2014). Reads were imported to QIIME2 for dereplication and chimeric reads screened and filtered from the dataset. Operational taxonomic unit clustering was completed within QIIME2 version 2019.7.0 (Bolyen et al., 2018) at 85\% similarity to account for typical sequencing errors obtained from long-read sequencing. Taxonomy was assigned to reads using a pre-trained classifier on the SILVA 132 16S rRNA representative sequences. Data was imported into $R$ version 3.6.1 with qiime2R version 0.99 .13 (https://github.com/jbisanz/qiime2R) for visualisation and alpha diversity analysis using raw and rarefied data with the phyloseq version 1.30.0 (McMurdie and Holmes, 2013) package. Scripts for command line processing and analysis in R available in Supplementary Materials. Sequencing data has been deposited in the NCBI database Sequence Read Archive (SRA) under accession numbers PRJNA635359. 


\section{SUPPLEMENTARY TABLES}

\section{Supplementary Table 1.}

\begin{tabular}{c|ccc}
\hline Time (mins) & Flow rate $(\boldsymbol{\mu l} / \mathbf{m i n})$ & Buffer A (\%) & Buffer B (\%) \\
\hline 0 & 200 & 15 & 85 \\
0.1 & 200 & 15 & 85 \\
10 & 200 & 30 & 70 \\
13 & 200 & 85 & 30 \\
17 & 200 & 85 & 30 \\
17.5 & 200 & 15 & 85 \\
30 & 200 & 15 & 85 \\
\hline
\end{tabular}

Supplementary Table 2. Liquid chromatography (LC) separation gradient

Buffer A: 95:5 (v/v) HPLC $\mathrm{H}_{2} \mathrm{O}$ :Acetonitrile $\left(\mathrm{CH}_{3} \mathrm{CN}\right)$ with $20 \mathrm{mM}$ ammonium acetate $\left(\mathrm{NH}_{4} \mathrm{OAc}\right)$ $+20 \mathrm{mM}$ acetic acid $\left(\mathrm{CH}_{3} \mathrm{COOH}\right), \mathrm{pH}$ 5. Buffer B: $100 \%$ Acetonitrile $\left(\mathrm{CH}_{3} \mathrm{CN}\right)$. 


\section{Supplementary Table 2.}

\begin{tabular}{|c|c|c|c|c|c|c|}
\hline Metabolite_Q1_Q3 & $\begin{array}{l}\text { Q1 } \\
(\mathrm{m} / \mathrm{z})\end{array}$ & $\begin{array}{l}\mathbf{Q 3} \\
(\mathrm{m} / \mathbf{z})\end{array}$ & $\begin{array}{l}\text { DP } \\
(V)\end{array}$ & $\begin{array}{l}\mathbf{C E} \\
(\mathrm{V})\end{array}$ & $\begin{array}{l}\text { CXP } \\
\text { (V) }\end{array}$ & $\begin{array}{l}\text { RT } \\
\text { (mins) }\end{array}$ \\
\hline glutamine_0_0 & 147 & 44 & 51 & 73 & 10 & 13 \\
\hline glutamine_1_0 & 148 & 44 & 51 & 73 & 10 & 13 \\
\hline glutamine_1_1 & 148 & 45 & 51 & 73 & 10 & 13 \\
\hline glutamine 2 & 149 & 45 & 51 & 73 & 10 & 13 \\
\hline NA 000 & 124 & 78 & 70 & 25 & 10 & 4 \\
\hline NA_1_1 & 125 & 79 & 70 & 25 & 10 & 4 \\
\hline NaAD_0_0 & 665 & 428 & 139 & 35 & 38 & 17 \\
\hline $\mathrm{NaAD} 5500$ & 670 & 428 & 139 & 35 & 38 & 17 \\
\hline NaAD_6_0 & 671 & 428 & 139 & 35 & 38 & 17 \\
\hline NaAD_1_0 & 666 & 428 & 139 & 35 & 38 & 17 \\
\hline $\mathrm{NaAD}$ 5_5 & 670 & 433 & 139 & 35 & 38 & 17 \\
\hline NaAD_10_5 & 675 & 433 & 139 & 35 & 38 & 17 \\
\hline $\mathrm{NaAD} \_11 \_5$ & 676 & 433 & 139 & 35 & 38 & 17 \\
\hline NaAD_6_5 & 671 & 433 & 139 & 35 & 38 & 17 \\
\hline NaAD_6_6 & 671 & 434 & 139 & 35 & 38 & 17 \\
\hline $\mathrm{NaAD} \_11 \_6$ & 676 & 434 & 139 & 35 & 38 & 17 \\
\hline $\mathrm{NaAD} \_12 \_6$ & 677 & 434 & 139 & 35 & 38 & 17 \\
\hline NaAD_7_6 & 672 & 434 & 139 & 35 & 38 & 17 \\
\hline NaAD_1_1 & 666 & 429 & 139 & 35 & 38 & 17 \\
\hline NaAD_6_1 & 671 & 429 & 139 & 35 & 38 & 17 \\
\hline $\mathrm{NaAD}{ }^{-7}+1$ & 672 & 429 & 139 & 35 & 38 & 17 \\
\hline $\mathrm{NaAD} 22 \_1$ & 667 & 429 & 139 & 35 & 38 & 17 \\
\hline $\mathrm{NaAD}+7 \_7$ & 672 & 435 & 139 & 35 & 38 & 17 \\
\hline NAD_0_0 & 664 & 428 & 33 & 36 & 31 & 17 \\
\hline NAD_5_0 & 669 & 428 & 33 & 36 & 31 & 17 \\
\hline NAD_6_0 & 670 & 428 & 33 & 36 & 31 & 17 \\
\hline NAD_7_0 & 671 & 428 & 33 & 36 & 31 & 17 \\
\hline NAD_1_0 & 665 & 428 & 33 & 36 & 31 & 17 \\
\hline NAD_2_0 & 666 & 428 & 33 & 36 & 31 & 17 \\
\hline NAD_5_5 & 669 & 433 & 33 & 36 & 31 & 17 \\
\hline NAD_6_6 & 670 & 434 & 33 & 36 & 31 & 17 \\
\hline NAD_7_7 & 671 & 435 & 33 & 36 & 31 & 17 \\
\hline NAD_1_1 & 665 & 429 & 33 & 36 & 31 & 17 \\
\hline NAD_2_2 & 666 & 430 & 33 & 36 & 31 & 17 \\
\hline NAD_10_5 & 674 & 433 & 33 & 36 & 31 & 17 \\
\hline NAD_11_5 & 675 & 433 & 33 & 36 & 31 & 17 \\
\hline NAD_12_5 & 676 & 433 & 33 & 36 & 31 & 17 \\
\hline NAD_6_1 & 670 & 429 & 33 & 36 & 31 & 17 \\
\hline NAD_7_1 & 671 & 429 & 33 & 36 & 31 & 17 \\
\hline NAD_8_1 & 672 & 429 & 33 & 36 & 31 & 17 \\
\hline NAD_11_6 & 675 & 434 & 33 & 36 & 31 & 17 \\
\hline NAD_12_6 & 676 & 434 & 33 & 36 & 31 & 17 \\
\hline NAD_13_6 & 677 & 434 & 33 & 36 & 31 & 17 \\
\hline NAM_0_0 & 123 & 80 & 80 & 30 & 25 & 2 \\
\hline
\end{tabular}




\begin{tabular}{|l|l|l|l|l|l|l|}
\hline NAM_1_1 & 124 & 81 & 80 & 30 & 25 & 2 \\
\hline NAM_2_1 & 125 & 81 & 80 & 30 & 25 & 2 \\
\hline NAM_1_0 & 124 & 80 & 80 & 30 & 25 & 2 \\
\hline NaMN_0_0 & 336 & 124 & 66 & 28 & 11 & 19 \\
\hline NaMN_5_0 & 341 & 124 & 66 & 28 & 11 & 19 \\
\hline NaMN_6_1 & 342 & 125 & 66 & 28 & 11 & 19 \\
\hline NaMN_1_1 & 337 & 125 & 66 & 28 & 11 & 19 \\
\hline NaR_0_0 & 256 & 124 & 41 & 27 & 6 & 10 \\
\hline NaR_5_0 & 261 & 124 & 41 & 27 & 6 & 10 \\
\hline NaR_6_1 & 262 & 125 & 41 & 27 & 6 & 10 \\
\hline NaR_1_1 & 257 & 125 & 41 & 27 & 6 & 10 \\
\hline NMN_0_0 & 335 & 123 & 48 & 24 & 11 & 19 \\
\hline NMN_5_0 & 340 & 123 & 48 & 24 & 11 & 19 \\
\hline NMN_6_1 & 341 & 124 & 48 & 24 & 11 & 19 \\
\hline NMN_7_2 & 342 & 125 & 48 & 24 & 11 & 19 \\
\hline NMN_1_1 & 336 & 124 & 48 & 24 & 11 & 19 \\
\hline NMN_2_2 & 337 & 125 & 48 & 24 & 11 & 19 \\
\hline NR_0_0 & 255 & 123 & 64 & 30 & 13 & 11 \\
\hline NR_5_0 & 260 & 123 & 64 & 30 & 13 & 11 \\
\hline NR_6_1 & 261 & 124 & 64 & 30 & 13 & 11 \\
\hline NR_7_2 & 262 & 125 & 64 & 30 & 13 & 11 \\
\hline NR_1_1 & 256 & 124 & 64 & 30 & 13 & 11 \\
\hline NR_2_2 & 257 & 125 & 64 & 30 & 13 & 11 \\
\hline Thymine-d4 & 129 & 42 & -115 & -52 & -11 & 2 \\
\hline CSA & 231 & 80 & -170 & -40 & -13 & 2 \\
\hline MES & 196 & 100 & 140 & 31 & 25 & 4 \\
\hline & & & & & & \\
\hline & 25 & & & & 11 \\
\hline
\end{tabular}

Supplementary Table 2. MRM transitions and MS parameters of $\mathrm{NAD}^{+}$metabolites and MRM internal standards (Thymidine d4, CSA, MES). Q1: parent ion, Q3: fragment ion, MRM: multiple reaction monitoring, DP: declustering potential, CE: collision energy, CXP: collision cell exit potential. 


\section{References}

Belenky, P., Christensen, K.C., Gazzaniga, F., Pletnev, A.A., and Brenner, C. (2009). Nicotinamide riboside and nicotinic acid riboside salvage in fungi and mammals. Quantitative basis for Urh1 and purine nucleoside phosphorylase function in NAD+ metabolism. J Biol Chem 284, 158-164.

Berger, F., Lau, C., Dahlmann, M., and Ziegler, M. (2005). Subcellular compartmentation and differential catalytic properties of the three human nicotinamide mononucleotide adenylyltransferase isoforms. Journal of Biological Chemistry 280, 36334-36341.

Bertoldo, M.J., Listijono, D.R., Ho, W.J., Riepsamen, A.H., Goss, D.M., Richani, D., Jin, X.L., Mahbub, S., Campbell, J.M., Habibalahi, A., et al. (2020). NAD(+) Repletion Rescues Female Fertility during Reproductive Aging. Cell Rep 30, 1670-1681 e1677.

Bieganowski, P., and Brenner, C. (2004). Discoveries of nicotinamide riboside as a nutrient and conserved NRK genes establish a Preiss-Handler independent route to NAD + in fungi and humans. Cell 117, 495-502.

Bolger, A.M., Lohse, M., and Usadel, B. (2014). Trimmomatic: a flexible trimmer for Illumina sequence data. Bioinformatics 30, 2114-2120.

Bolyen, E., Rideout, J.R., Dillon, M.R., Bokulich, N.A., Abnet, C., Al-Ghalith, G.A., Alexander, H., Alm, E.J., Arumugam, M., and Asnicar, F. (2018). QIIME 2: Reproducible, interactive, scalable, and extensible microbiome data science (PeerJ Preprints).

Braidy, N., Guillemin, G.J., Mansour, H., Chan-Ling, T., Poljak, A., and Grant, R. (2011). Age related changes in NAD + metabolism oxidative stress and Sirt1 activity in wistar rats. PloS one 6, e19194.

Camacho-Pereira, J., Tarragó, M.G., Chini, C.C., Nin, V., Escande, C., Warner, G.M., Puranik, A.S., Schoon, R.A., Reid, J.M., and Galina, A. (2016). CD38 dictates age-related NAD decline and mitochondrial dysfunction through an SIRT3-dependent mechanism. Cell metabolism 23, 11271139.

Cantó, C., Houtkooper, R.H., Pirinen, E., Youn, D.Y., Oosterveer, M.H., Cen, Y., FernandezMarcos, P.J., Yamamoto, H., Andreux, P.A., and Cettour-Rose, P. (2012). The NAD+ precursor nicotinamide riboside enhances oxidative metabolism and protects against high-fat diet-induced obesity. Cell metabolism 15, 838-847.

Chen, X.C., Hentz, N.G., Hubbard, F., Meier, T.I., Sittampalam, S., and Zhao, G. (2002). Development of a fluorescence resonance energy transfer assay for measuring the activity of Streptococcus pneumoniae DNA ligase, an enzyme essential for DNA replication, repair, and recombination. Analytical biochemistry 309, 232-240. 
bioRxiv preprint doi: https://doi.org/10.1101/2020.09.10.289561; this version posted September 11, 2020. The copyright holder for this preprint (which was not certified by peer review) is the author/funder, who has granted bioRxiv a license to display the preprint in perpetuity. It is made available under aCC-BY-ND 4.0 International license.

Chowdhury, M.K., Wu, L.E., Coleman, J.L., Smith, N.J., Morris, M.J., Shepherd, P.R., and Smith, G.C. (2016). Niclosamide blocks glucagon phosphorylation of Ser552 on beta-catenin in primary rat hepatocytes via PKA signalling. Biochem J 473, 1247-1255.

Clement, J., Wong, M., Poljak, A., Sachdev, P., and Braidy, N. (2019). The plasma NAD+ metabolome is dysregulated in "normal" aging. Rejuvenation research 22, 121-130.

Das, A., Huang, G.X., Bonkowski, M.S., Longchamp, A., Li, C., Schultz, M.B., Kim, L.-J., Osborne, B., Joshi, S., and Lu, Y. (2018). Impairment of an endothelial NAD+-H2S signaling network is a reversible cause of vascular aging. Cell 173, 74-89. e20.

de Murcia, G., and de Murcia, J.M. (1994). Poly (ADP-ribose) polymerase: a molecular nicksensor. Trends in biochemical sciences 19, 172-176.

Emanuelli, M., Carnevali, F., Saccucci, F., Pierella, F., Amici, A., Raffaelli, N., and Magni, G. (2001). Molecular cloning, chromosomal localization, tissue mRNA levels, bacterial expression, and enzymatic properties of human NMN adenylyltransferase. Journal of Biological Chemistry 276, 406-412.

Frederick, D.W., Loro, E., Liu, L., Davila Jr, A., Chellappa, K., Silverman, I.M., Quinn III, W.J., Gosai, S.J., Tichy, E.D., and Davis, J.G. (2016). Loss of NAD homeostasis leads to progressive and reversible degeneration of skeletal muscle. Cell metabolism 24, 269-282.

Frothingham, R., Meeker-O'Connell, W.A., Talbot, E., George, J.W., and Kreuzer, K.N. (1996). Identification, cloning, and expression of the Escherichia coli pyrazinamidase and nicotinamidase gene, pncA. Antimicrobial agents and chemotherapy 40, 1426-1431.

Galeazzi, L., Bocci, P., Amici, A., Brunetti, L., Ruggieri, S., Romine, M., Reed, S., Osterman, A.L., Rodionov, D.A., and Sorci, L. (2011). Identification of nicotinamide mononucleotide deamidase of the bacterial pyridine nucleotide cycle reveals a novel broadly conserved amidohydrolase family. Journal of Biological Chemistry 286, 40365-40375.

Garavaglia, S., D'Angelo, I., Emanuelli, M., Carnevali, F., Pierella, F., Magni, G., and Rizzi, M. (2002). Structure of human NMN adenylyltransferase a key nuclear enzyme for NAD homeostasis. Journal of Biological Chemistry 277, 8524-8530.

Gazzaniga, F., Stebbins, R., Chang, S.Z., McPeek, M.A., and Brenner, C. (2009). Microbial NAD metabolism: lessons from comparative genomics. Microbiol Mol Biol Rev 73, 529-541.

Geider, K. (1972). DNA Synthesis in Nucleotide-Permeable Escherichia coli Cells: The Effects of Nucleotide Analogues on DNA Synthesis. European journal of biochemistry 27, 554-563.

Gerdts, J., Brace, E.J., Sasaki, Y., DiAntonio, A., and Milbrandt, J. (2015). SARM1 activation triggers axon degeneration locally via $\mathrm{NAD}(+)$ destruction. Science 348, 453-457.

Gomes, A.P., Price, N.L., Ling, A.J., Moslehi, J.J., Montgomery, M.K., Rajman, L., White, J.P., Teodoro, J.S., Wrann, C.D., and Hubbard, B.P. (2013). Declining NAD+ induces a pseudohypoxic state disrupting nuclear-mitochondrial communication during aging. Cell 155, 1624-1638. 
bioRxiv preprint doi: https://doi.org/10.1101/2020.09.10.289561; this version posted September 11, 2020. The copyright holder for this preprint (which was not certified by peer review) is the author/funder, who has granted bioRxiv a license to display the preprint in perpetuity. It is made available under aCC-BY-ND 4.0 International license.

Grozio, A., Mills, K.F., Yoshino, J., Bruzzone, S., Sociali, G., Tokizane, K., Lei, H.C., Cunningham, R., Sasaki, Y., and Migaud, M.E. (2019). Slc12a8 is a nicotinamide mononucleotide transporter. Nature metabolism 1, 47.

Haigis, M.C., and Sinclair, D.A. (2010). Mammalian sirtuins: biological insights and disease relevance. Annual Review of Pathology: Mechanisms of Disease 5, 253-295.

Hara, N., Osago, H., Hiyoshi, M., Kobayashi-Miura, M., and Tsuchiya, M. (2019). Quantitative analysis of the effects of nicotinamide phosphoribosyltransferase induction on the rates of NAD+ synthesis and breakdown in mammalian cells using stable isotope-labeling combined with mass spectrometry. PLoS One 14, e0214000.

Imai, S.-I., Armstrong, C.M., Kaeberlein, M., and Guarente, L. (2000). Transcriptional silencing and longevity protein Sir2 is an NAD-dependent histone deacetylase. Nature 403, 795.

Jukarainen, S., Heinonen, S., Rämö, J.T., Rinnankoski-Tuikka, R., Rappou, E., Tummers, M., Muniandy, M., Hakkarainen, A., Lundbom, J., and Lundbom, N. (2016). Obesity is associated with low NAD+/SIRT pathway expression in adipose tissue of BMI-discordant monozygotic twins. The Journal of Clinical Endocrinology 101, 275-283.

Kim, M.Y., Zhang, T., and Kraus, W.L. (2005). Poly (ADP-ribosyl) ation by PARP-1:PARlaying'NAD+ into a nuclear signal. Genes \& development 19, 1951-1967.

Liu, L., Su, X., Quinn III, W.J., Hui, S., Krukenberg, K., Frederick, D.W., Redpath, P., Zhan, L., Chellappa, K., and White, E. (2018). Quantitative analysis of NAD synthesis-breakdown fluxes. Cell metabolism 27, 1067-1080. e1065.

Martens, C.R., Denman, B.A., Mazzo, M.R., Armstrong, M.L., Reisdorph, N., McQueen, M.B., Chonchol, M., and Seals, D.R. (2018). Chronic nicotinamide riboside supplementation is welltolerated and elevates NAD+ in healthy middle-aged and older adults. Nature communications 9 , 1286.

Massudi, H., Grant, R., Braidy, N., Guest, J., Farnsworth, B., and Guillemin, G.J. (2012). Ageassociated changes in oxidative stress and NAD+ metabolism in human tissue. PloS one 7, e42357.

McMurdie, P.J., and Holmes, S. (2013). phyloseq: an R package for reproducible interactive analysis and graphics of microbiome census data. PloS one 8 .

Mills, K.F., Yoshida, S., Stein, L.R., Grozio, A., Kubota, S., Sasaki, Y., Redpath, P., Migaud, M.E., Apte, R.S., and Uchida, K. (2016). Long-term administration of nicotinamide mononucleotide mitigates age-associated physiological decline in mice. Cell metabolism 24, 795-806.

Okabe, K., Yaku, K., Tobe, K., and Nakagawa, T. (2019). Implications of altered NAD metabolism in metabolic disorders. Journal of biomedical science 26, 34.

Olivera, B.M., and Lehman, I. (1967). Diphosphopyridine nucleotide: a cofactor for the polynucleotide-joining enzyme from Escherichia coli. Proceedings of the National Academy of Sciences of the United States of America 57, 1700. 
bioRxiv preprint doi: https://doi.org/10.1101/2020.09.10.289561; this version posted September 11, 2020. The copyright holder for this preprint (which was not certified by peer review) is the author/funder, who has granted bioRxiv a license to display the preprint in perpetuity. It is made available under aCC-BY-ND 4.0 International license.

Petrack, B., Greengard, P., Craston, A., and Sheppy, F. (1965). Nicotinamide Deamidase from Mammalian Liver. J Biol Chem 240, 1725-1730.

Preiss, J., and Handler, P. (1958a). Biosynthesis of diphosphopyridine nucleotide II. Enzymatic aspects. Journal of Biological Chemistry 233, 493-500.

Preiss, J., and Handler, P. (1958b). Biosynthesis of diphosphopyridine nucleotide. I. Identification of intermediates. J Biol Chem 233, 488-492.

Raffaelli, N., Sorci, L., Amici, A., Emanuelli, M., Mazzola, F., and Magni, G. (2002). Identification of a novel human nicotinamide mononucleotide adenylyltransferase. Biochemical and biophysical research communications 297, 835-840.

Rahman, M., Hasan, M.R., Oba, T., and Shimizu, K. (2006). Effect of rpoS gene knockout on the metabolism of Escherichia coli during exponential growth phase and early stationary phase based on gene expressions, enzyme activities and intracellular metabolite concentrations. Biotechnology and bioengineering $94,585-595$.

Rajman, L., Chwalek, K., and Sinclair, D.A. (2018). Therapeutic potential of NAD-boosting molecules: the in vivo evidence. Cell metabolism 27, 529-547.

Ratajczak, J., Joffraud, M., Trammell, S.A., Ras, R., Canela, N., Boutant, M., Kulkarni, S.S., Rodrigues, M., Redpath, P., and Migaud, M.E. (2016a). NRK1 controls nicotinamide mononucleotide and nicotinamide riboside metabolism in mammalian cells. Nature communications 7,13103 .

Ratajczak, J., Joffraud, M., Trammell, S.A., Ras, R., Canela, N., Boutant, M., Kulkarni, S.S., Rodrigues, M., Redpath, P., Migaud, M.E., et al. (2016b). NRK1 controls nicotinamide mononucleotide and nicotinamide riboside metabolism in mammalian cells. Nat Commun 7, 13103.

Revollo, J.R., Grimm, A.A., and Imai, S.-i. (2004). The NAD biosynthesis pathway mediated by nicotinamide phosphoribosyltransferase regulates Sir2 activity in mammalian cells. Journal of Biological Chemistry 279, 50754-50763.

Ryu, D., Zhang, H., Ropelle, E.R., Sorrentino, V., Mázala, D.A., Mouchiroud, L., Marshall, P.L., Campbell, M.D., Ali, A.S., and Knowels, G.M. (2016). NAD+ repletion improves muscle function in muscular dystrophy and counters global PARylation. Science translational medicine 8, 361ra139361 ra139.

Sasaki, Y., Nakagawa, T., Mao, X., DiAntonio, A., and Milbrandt, J. (2016). NMNAT1 inhibits axon degeneration via blockade of SARM1-mediated NAD $(+)$ depletion. Elife 5.

Schultz, M.B., and Sinclair, D.A. (2016). Why NAD+ declines during aging: It's destroyed. Cell metabolism 23, 965-966.

Schweiger, M., Hennig, K., Lerner, F., Niere, M., Hirsch-Kauffmann, M., Specht, T., Weise, C., Oei, S.L., and Ziegler, M. (2001). Characterization of recombinant human nicotinamide mononucleotide adenylyl transferase (NMNAT), a nuclear enzyme essential for NAD synthesis. FEBS letters 492, 95-100. 
bioRxiv preprint doi: https://doi.org/10.1101/2020.09.10.289561; this version posted September 11, 2020. The copyright holder for this preprint (which was not certified by peer review) is the author/funder, who has granted bioRxiv a license to display the preprint in perpetuity. It is made available under aCC-BY-ND 4.0 International license.

Shats, I., Williams, J.G., Liu, J., Makarov, M.V., Wu, X., Lih, F.B., Deterding, L.J., Lim, C., Xu, X., Randall, T.A., et al. (2020). Bacteria Boost Mammalian Host NAD Metabolism by Engaging the Deamidated Biosynthesis Pathway. Cell Metab 31, 564-579 e567.

Shimoyama, M., Tanigawa, Y., Ito, T., Murashima, R., Ueda, I., and Tomoda, T. (1971). Nicotinamide deamidation by microorganisms in rat stomach. Journal of bacteriology 108, 191-195.

Tanigawa, Y., Shimoyama, M., Murashima, R., Ito, T., Yamaguchi, K., and Ueda, I. (1970). The role of microorganisms as a function of nicotinamide deamidation in rat stomach. Biochimica et Biophysica Acta (BBA)-General Subjects 201, 394-397.

Tarantini, S., Valcarcel-Ares, M.N., Toth, P., Yabluchanskiy, A., Tucsek, Z., Kiss, T., Hertelendy, P., Kinter, M., Ballabh, P., Sule, Z., et al. (2019). Nicotinamide mononucleotide (NMN) supplementation rescues cerebromicrovascular endothelial function and neurovascular coupling responses and improves cognitive function in aged mice. Redox Biol 24, 101192.

Trammell, S.A., Schmidt, M.S., Weidemann, B.J., Redpath, P., Jaksch, F., Dellinger, R.W., Li, Z., Abel, E.D., Migaud, M.E., and Brenner, C. (2016). Nicotinamide riboside is uniquely and orally bioavailable in mice and humans. Nature communications 7, 12948.

Ubeda, C., Taur, Y., Jenq, R.R., Equinda, M.J., Son, T., Samstein, M., Viale, A., Socci, N.D., Van Den Brink, M.R., and Kamboj, M. (2010). Vancomycin-resistant Enterococcus domination of intestinal microbiota is enabled by antibiotic treatment in mice and precedes bloodstream invasion in humans. The Journal of clinical investigation 120, 4332-4341.

Xiao, W., Wang, R.-S., Handy, D.E., and Loscalzo, J. (2018). NAD (H) and NADP (H) redox couples and cellular energy metabolism. Antioxidants \& redox signaling 28, 251-272.

Ying, W. (2008). NAD+/NADH and NADP+/NADPH in cellular functions and cell death: regulation and biological consequences. Antioxidants \& redox signaling 10, 179-206.

Yoshino, J., Baur, J.A., and Imai, S.-i. (2018). NAD+ intermediates: the biology and therapeutic potential of NMN and NR. Cell metabolism 27, 513-528.

Yoshino, J., Mills, K.F., Yoon, M.J., and Imai, S.-i. (2011). Nicotinamide mononucleotide, a key $\mathrm{NAD}+$ intermediate, treats the pathophysiology of diet-and age-induced diabetes in mice. Cell metabolism 14, 528-536.

Zhang, H., Ryu, D., Wu, Y., Gariani, K., Wang, X., Luan, P., D’Amico, D., Ropelle, E.R., Lutolf, M.P., and Aebersold, R. (2016). NAD+ repletion improves mitochondrial and stem cell function and enhances life span in mice. Science 352, 1436-1443.

Zhang, X., Kurnasov, O.V., Karthikeyan, S., Grishin, N.V., Osterman, A.L., and Zhang, H. (2003). Structural characterization of a human cytosolic NMN/NaMN adenylyltransferase and implication in human NAD biosynthesis. Journal of Biological Chemistry 278, 13503-13511.

Zimmerman, S.B., and Oshinsky, C.K. (1969). Enzymatic joining of deoxyribonucleic acid strands iii. Further purification of the deoxyribonucleic acid ligase from escherichia coli and multiple forms of the purified enzyme. Journal of Biological Chemistry 244, 4689-4695. 
bioRxiv preprint doi: https://doi.org/10.1101/2020.09.10.289561; this version posted September 11, 2020. The copyright holder for this preprint (which was not certified by peer review) is the author/funder, who has granted bioRxiv a license to display the preprint in perpetuity. It is made available under aCC-BY-ND 4.0 International license. 
bioRxiv preprint doi: https://doi.org/10.1101/2020.09.10.289561; this version posted September 11, 2020. The copyright holder for this

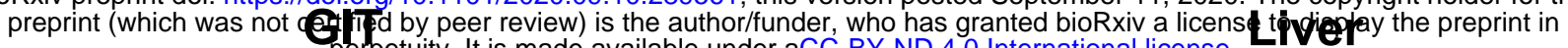
perpetuity. It is made available under aCC-BY-ND 4.0 International license.

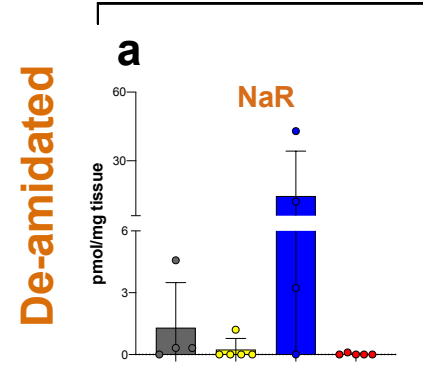

b c

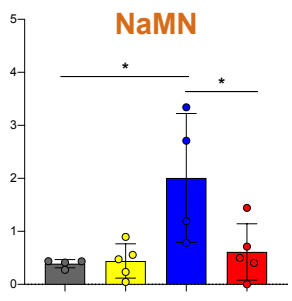

h

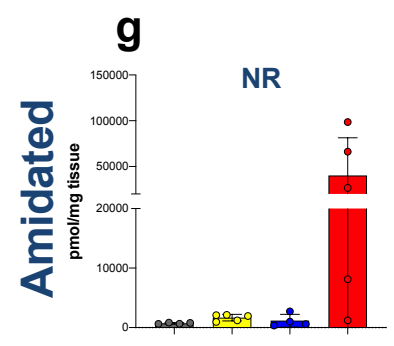

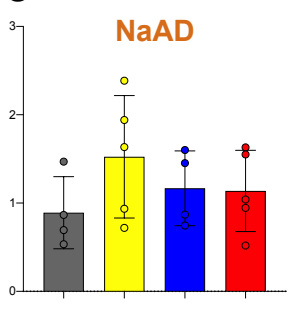

d

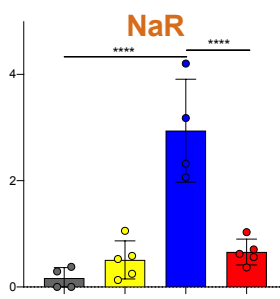

e
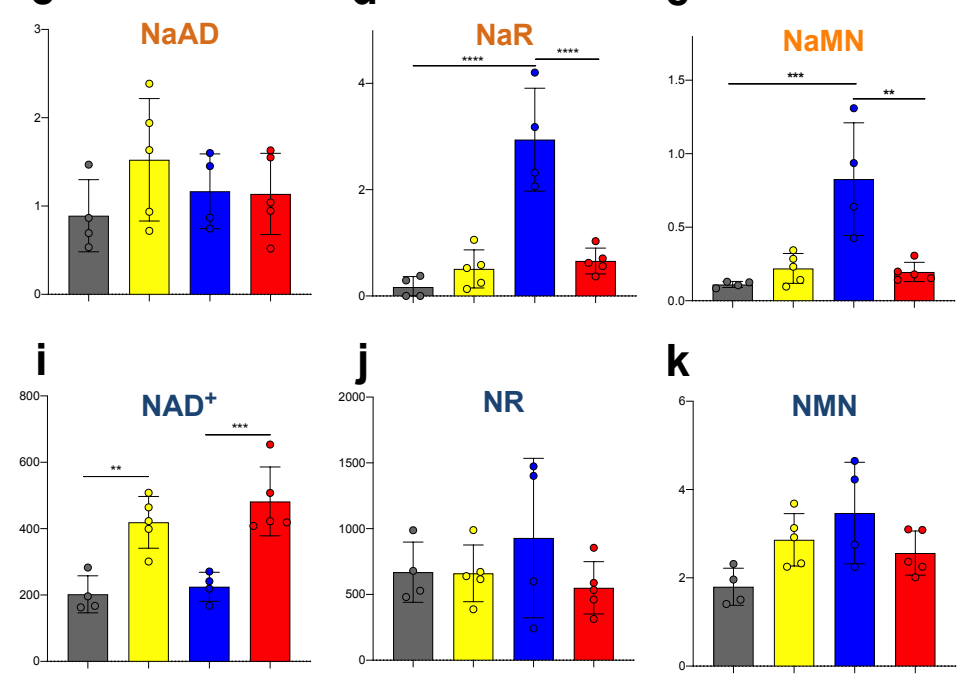

k

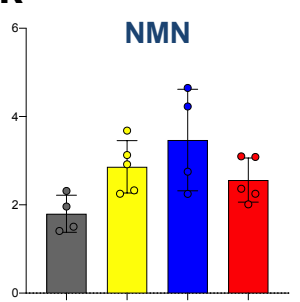

p

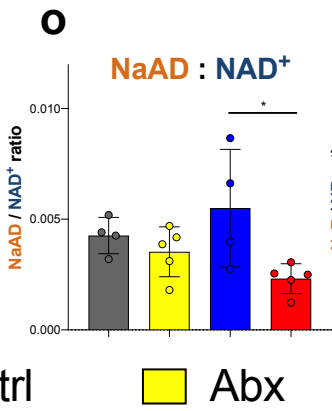

n

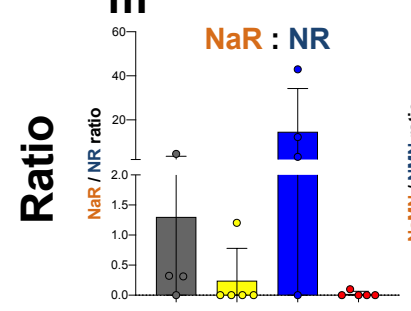

\section{Deamidated}

metabolites

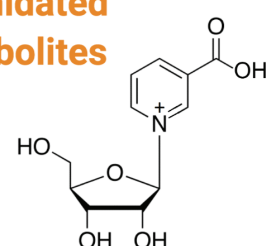

$\mathrm{NaR}$

NRK1/2

HO<smiles></smiles>

NMNAT1-3

ADP<smiles>CC(C)n1ccc(C=O)c1</smiles>

Amidated

$\mathrm{CN}-\mathrm{II} / \mathrm{III}$

metabolites

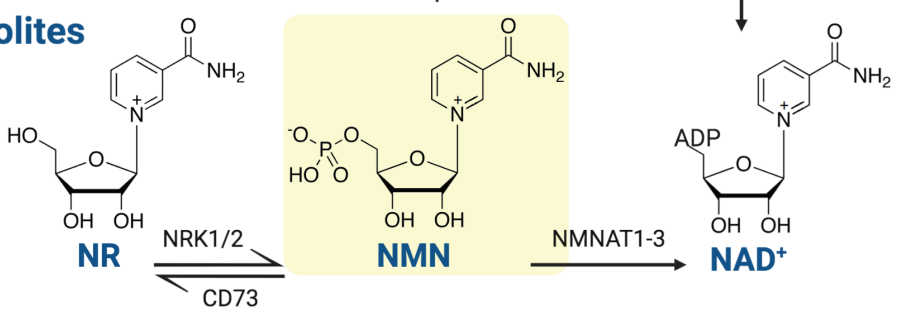


bioRxiv preprint doi: https://doi.org/10.1101/2020.09.10.289561; this version posted September 11, 2020. The copyright holder for this preprint (which was not certified by peer review) is the author/funder, who has granted bioRxiv a license to display the preprint in

a perpetuity. It is made available under aCC-BY-ND 4.0 International license.

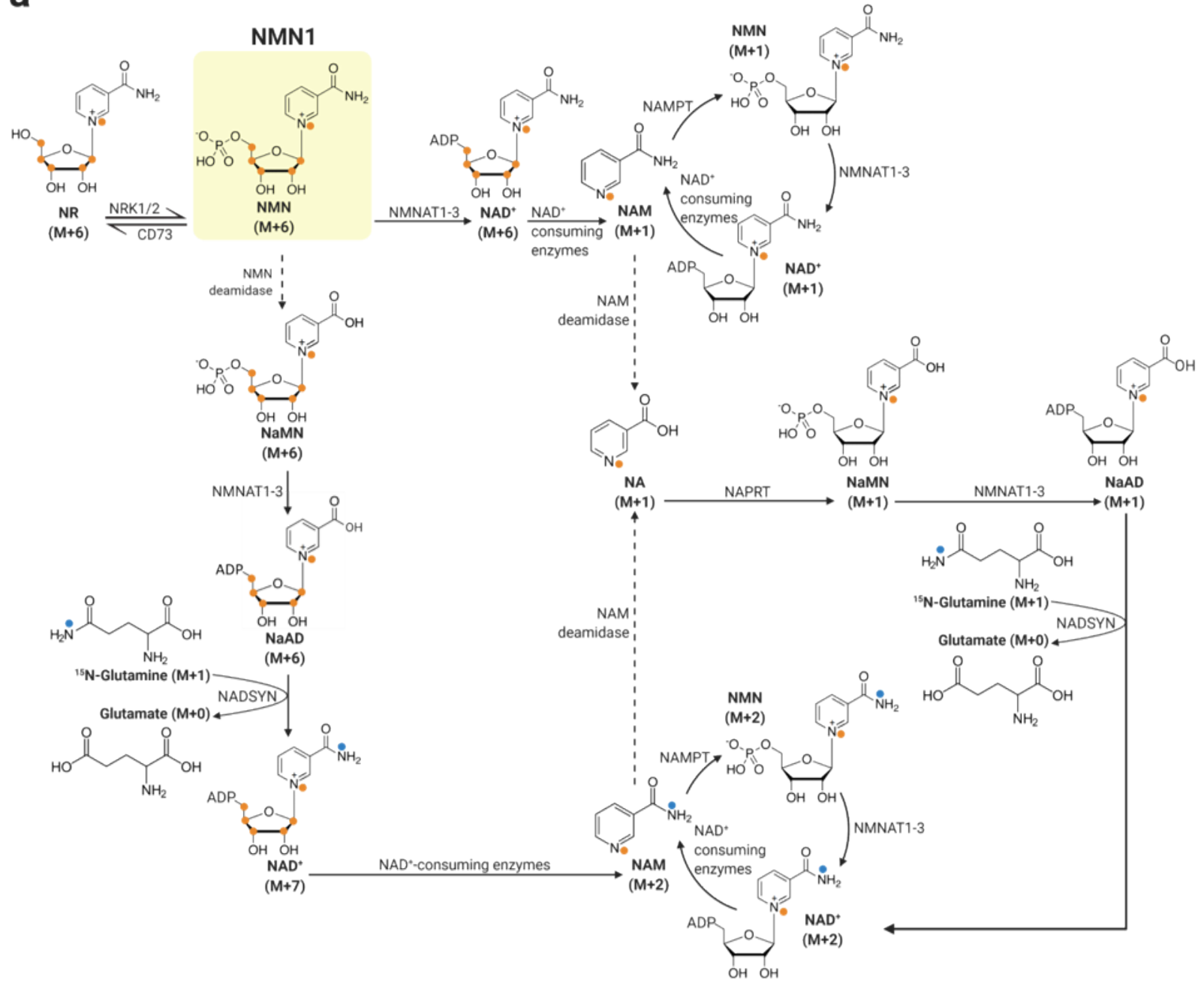

b

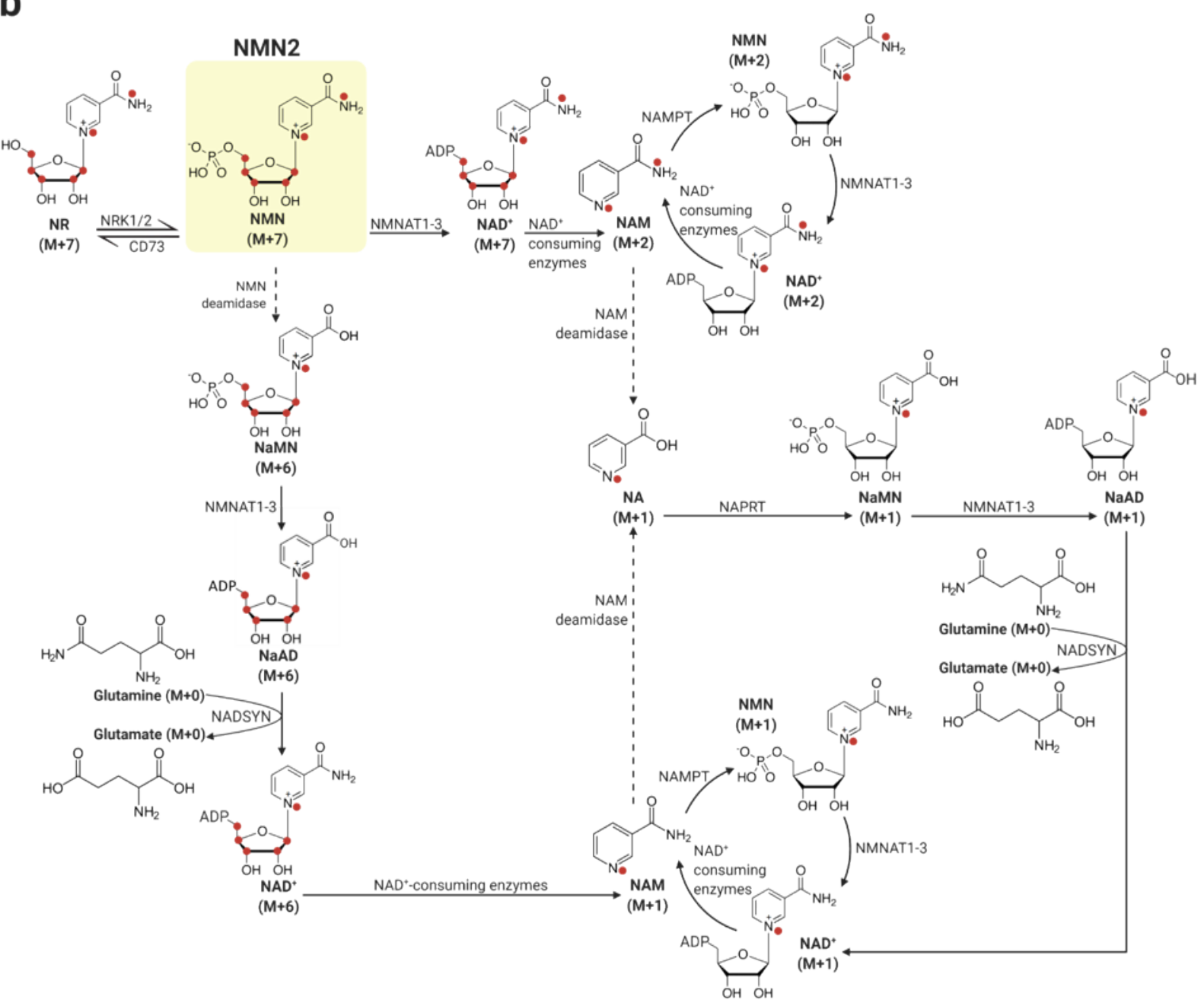




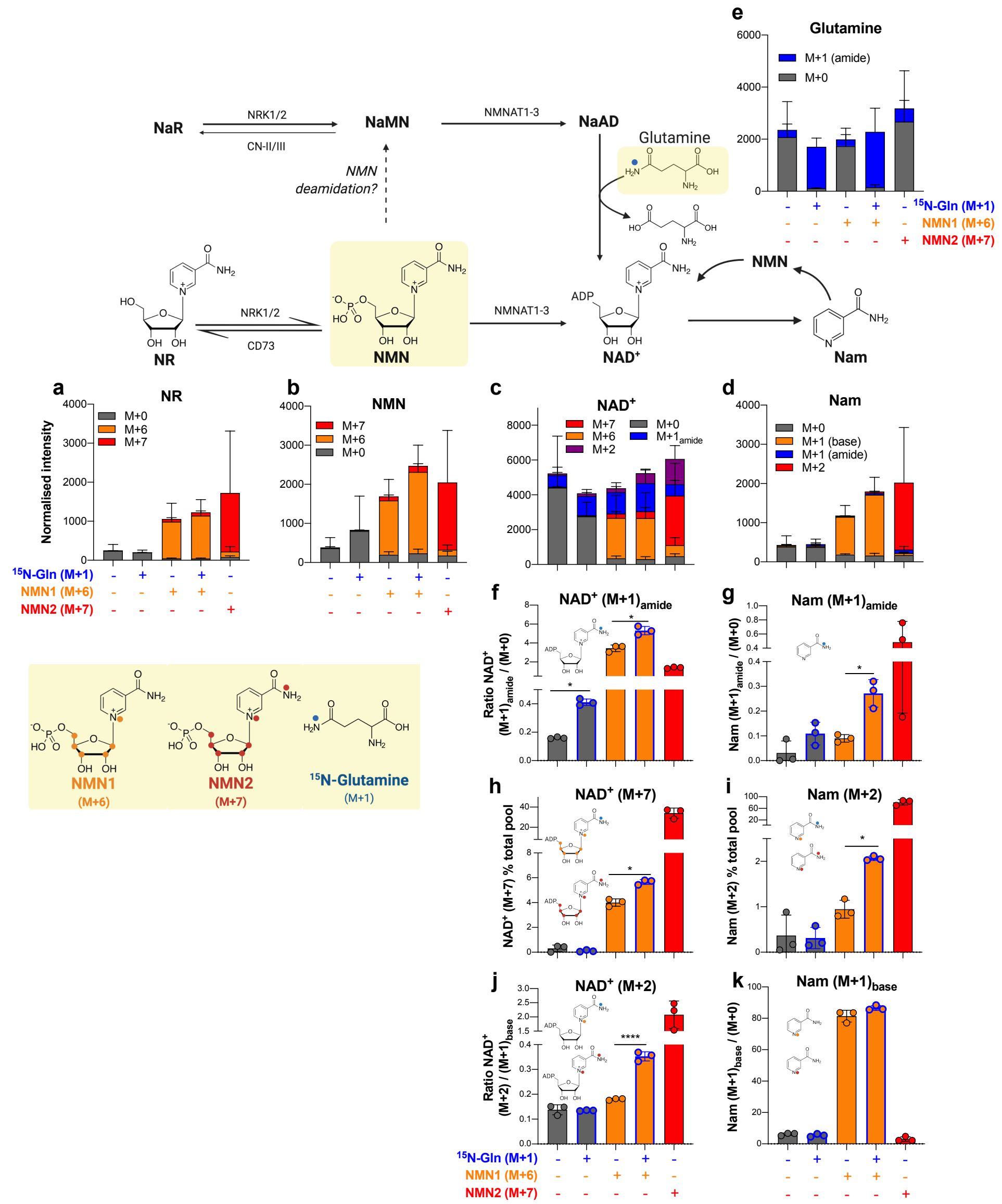




\section{Supernatant}

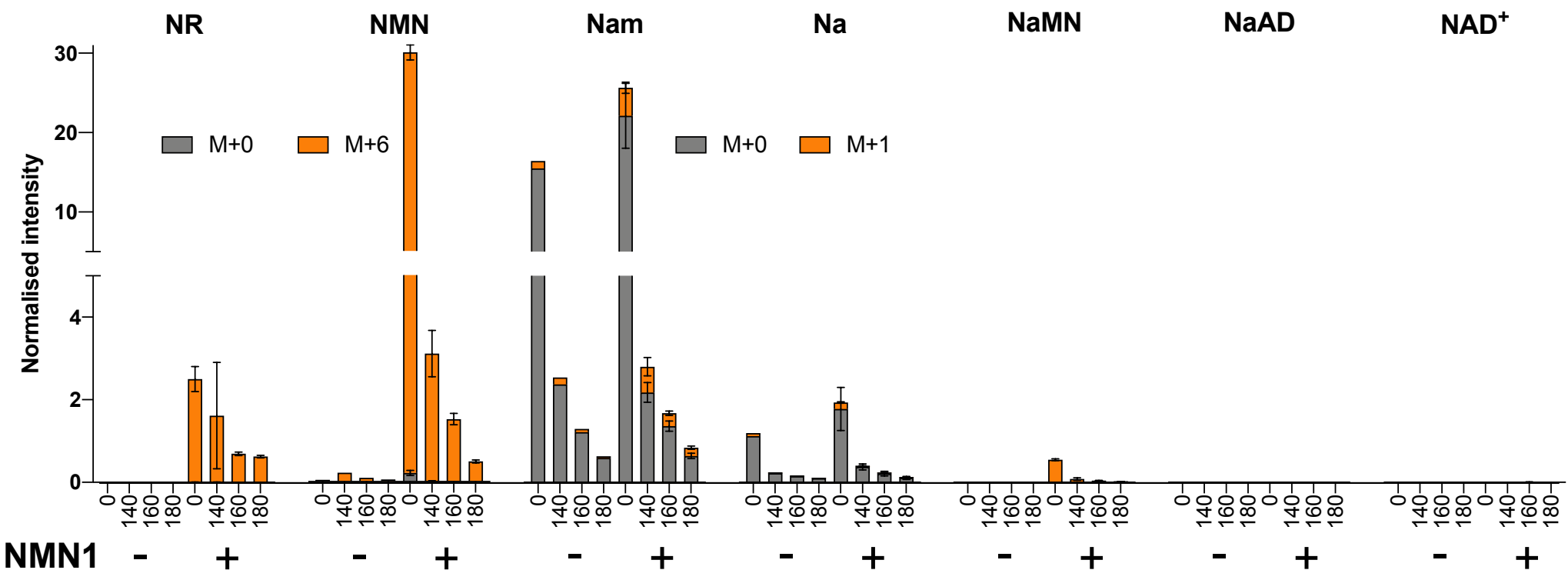

Extracellular

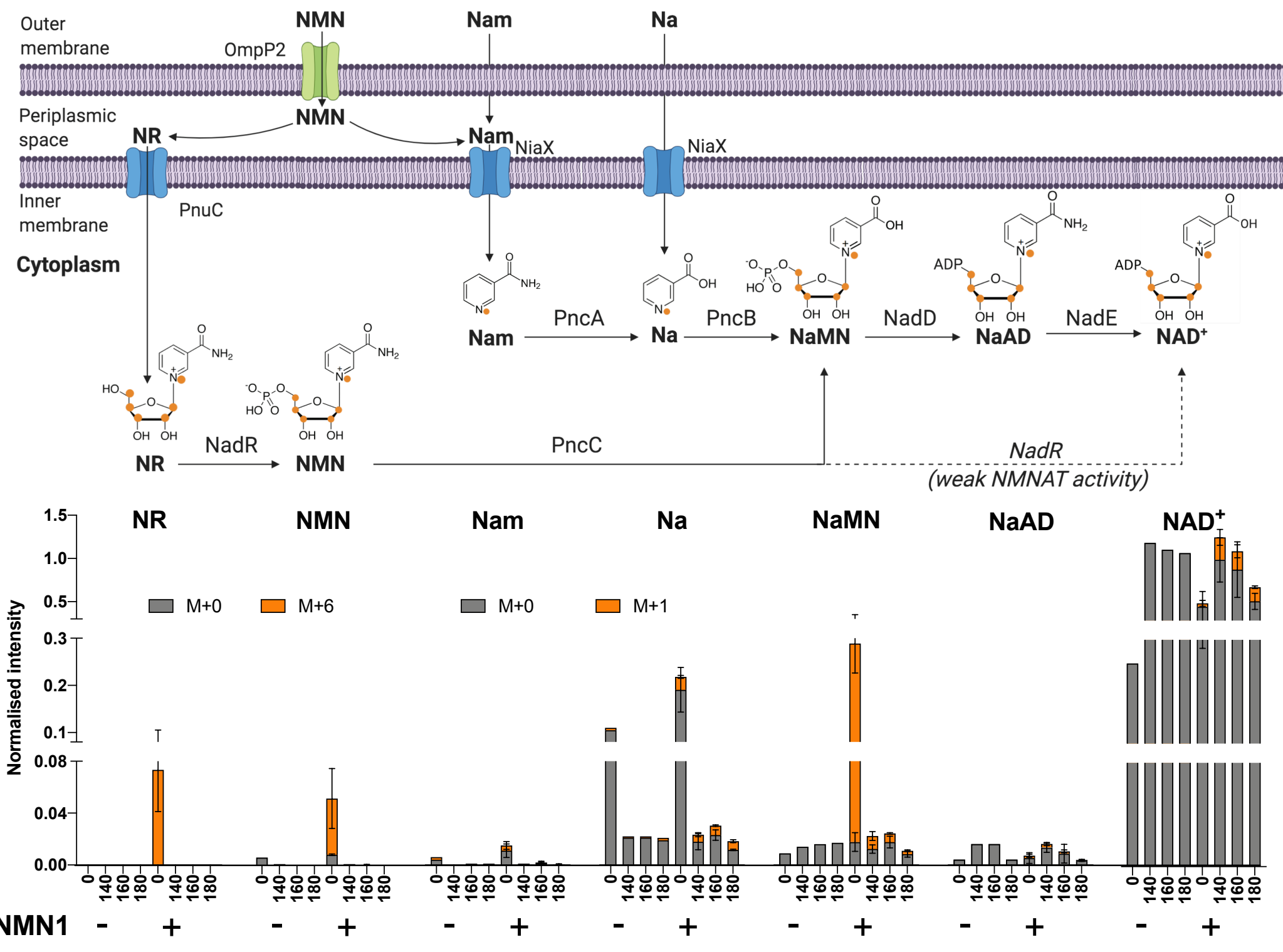




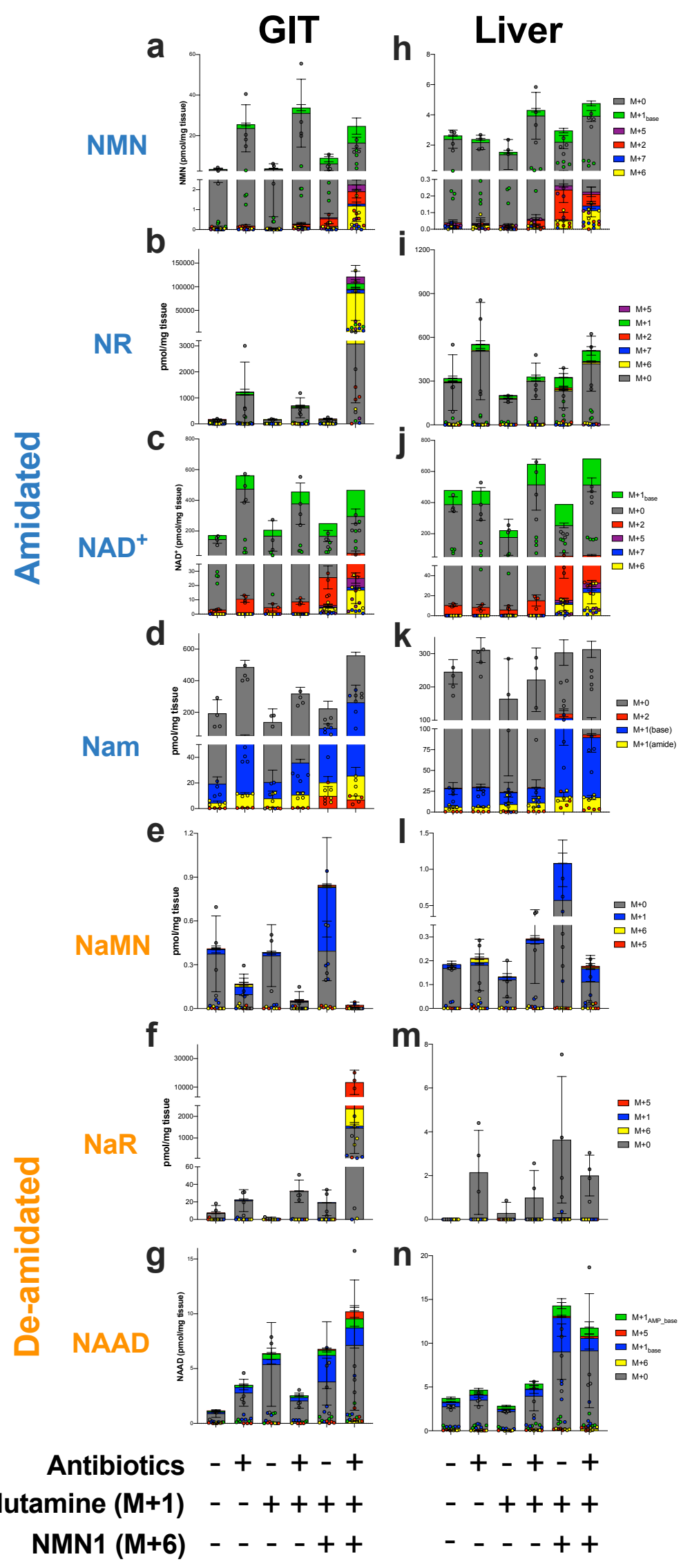


GIT

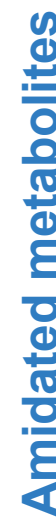

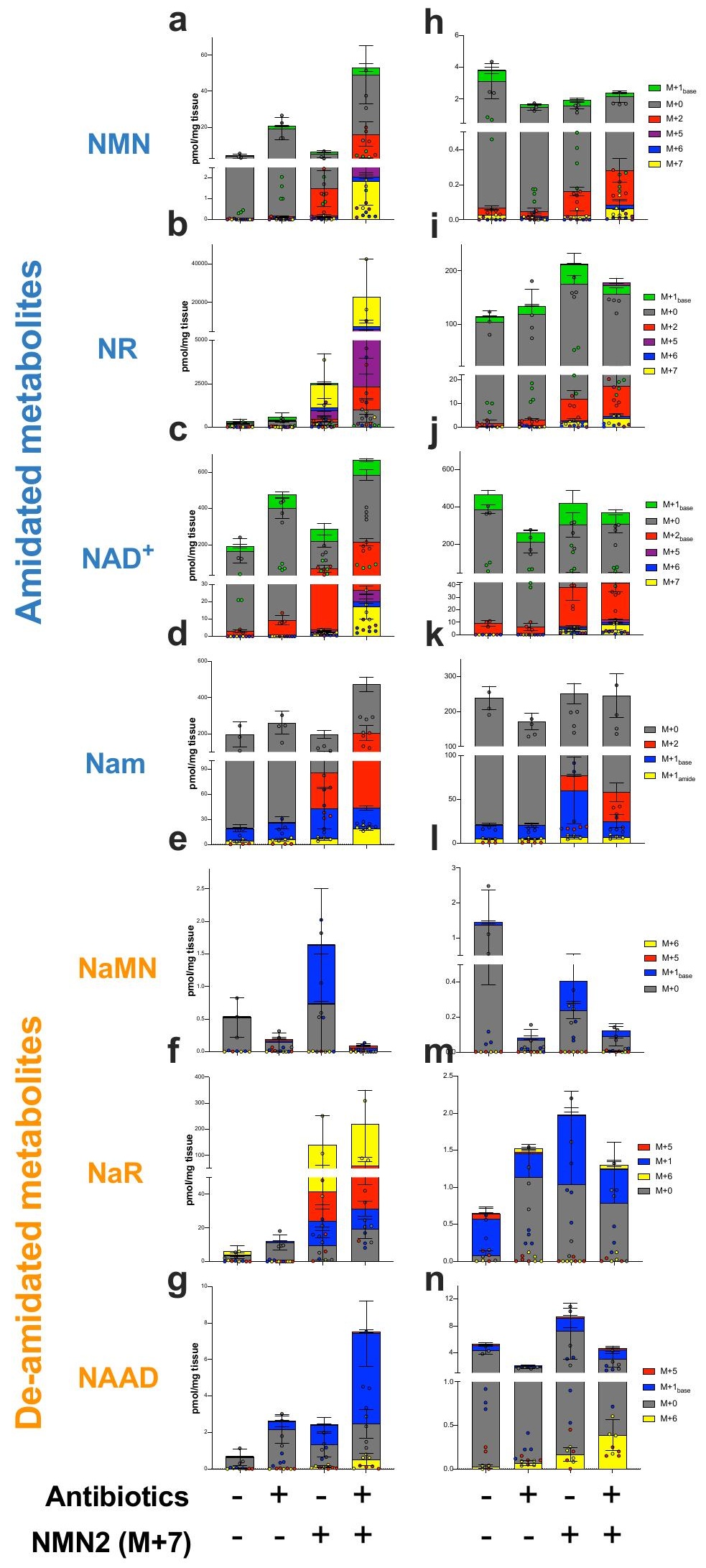


bioRxiv preprint doi: https://doi.org/10.1101/2020.09.10.289561; this version posted September 11,2020 . The copyright holder for this

a
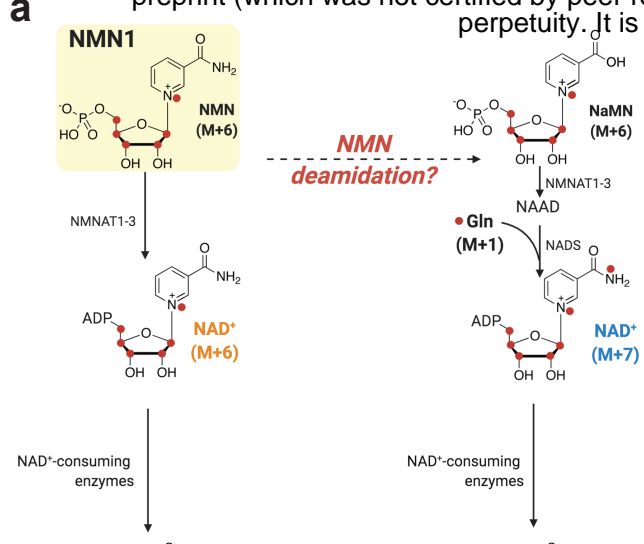

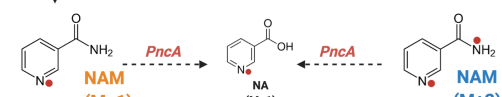

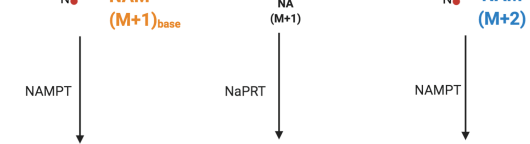

(M+1)

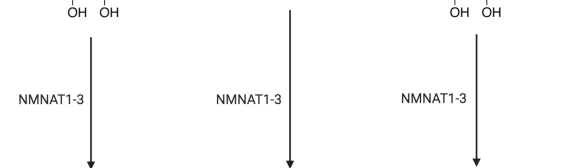

$\underbrace{\mathrm{OH}}_{\mathrm{OH}}(\mathrm{M}+1)_{\text {bose }}$

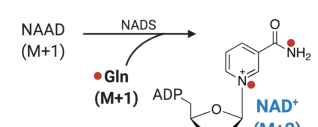

Amidated route

De-amidated route

j

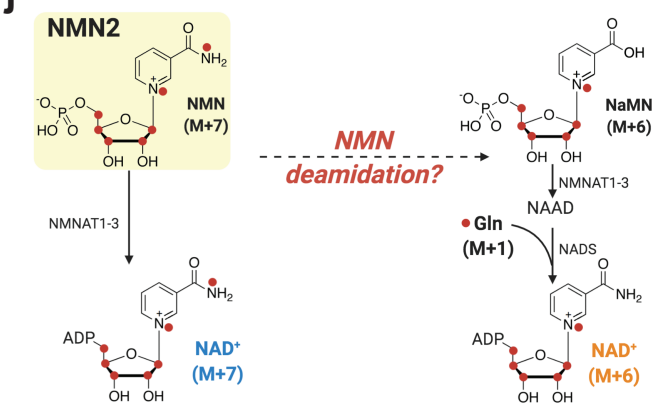

\begin{tabular}{c} 
NAD+consuming \\
enzymes \\
\hline
\end{tabular}
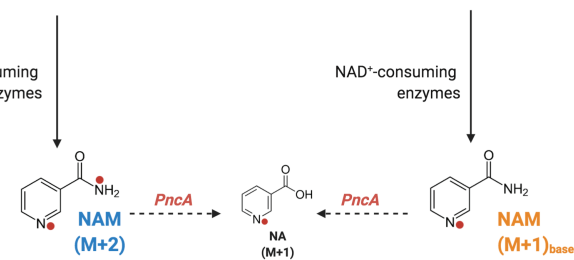

NAMPT
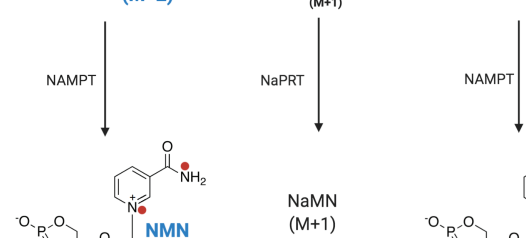

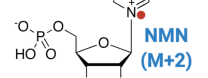

$(\mathrm{M}+1)$

$\mathrm{OH} \mathrm{OH}^{2}$
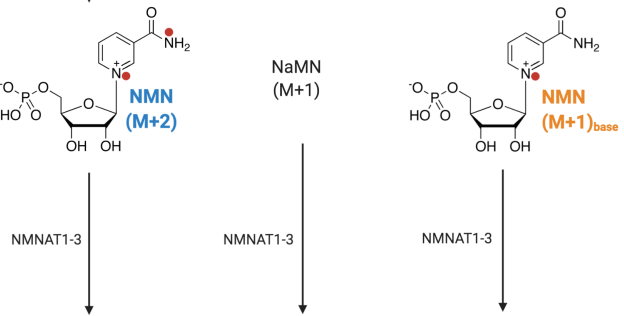

NMNAT1-3

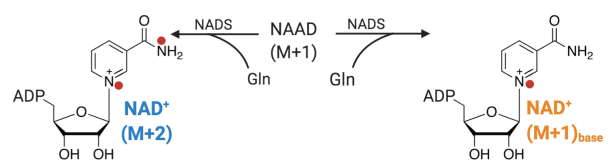

$\mathrm{NAD}^{+}$ (recycled)

Amidated route

De-amidated route

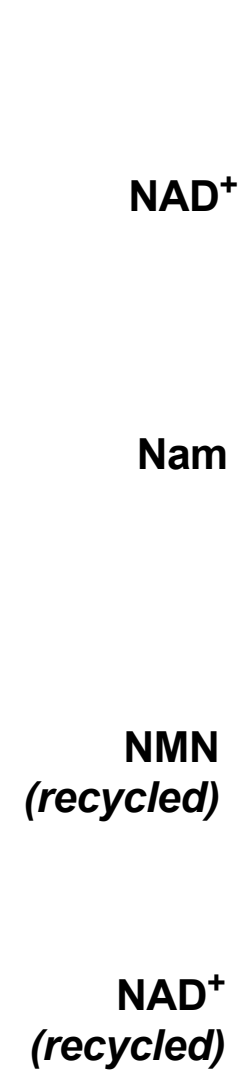

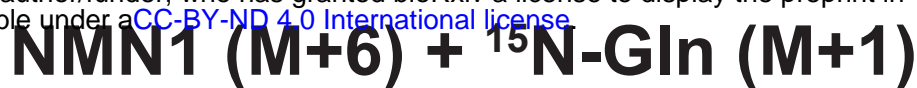

\section{GIT}

Liver

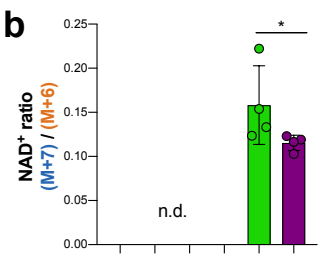

C
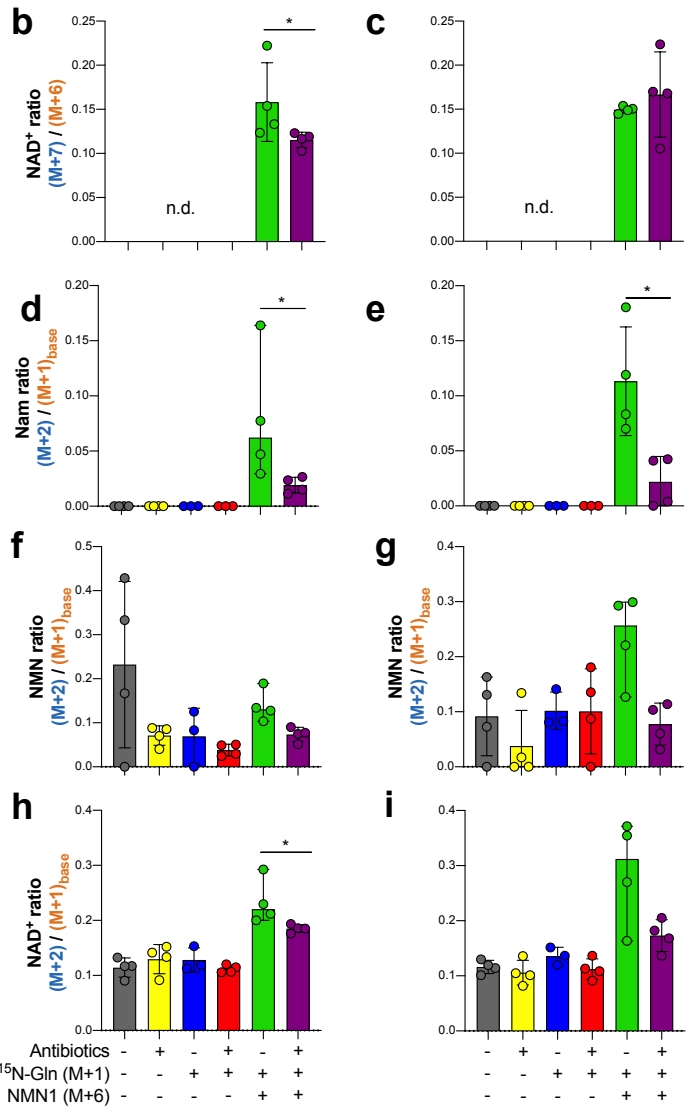

i

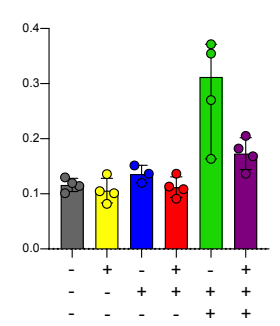

NMN2 $(M+7)$

k

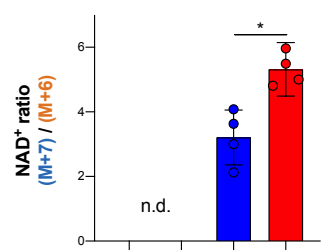

I

m

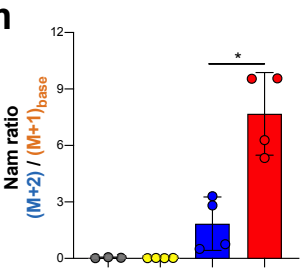

n

o

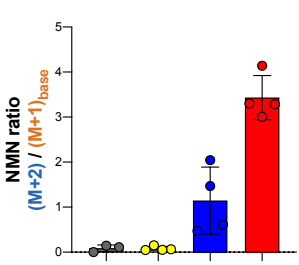

p

$\mathbf{q}$

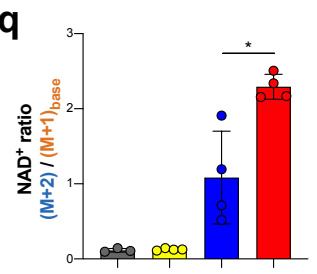

Antibiotics - + - + NMN2 $(M+7)-$
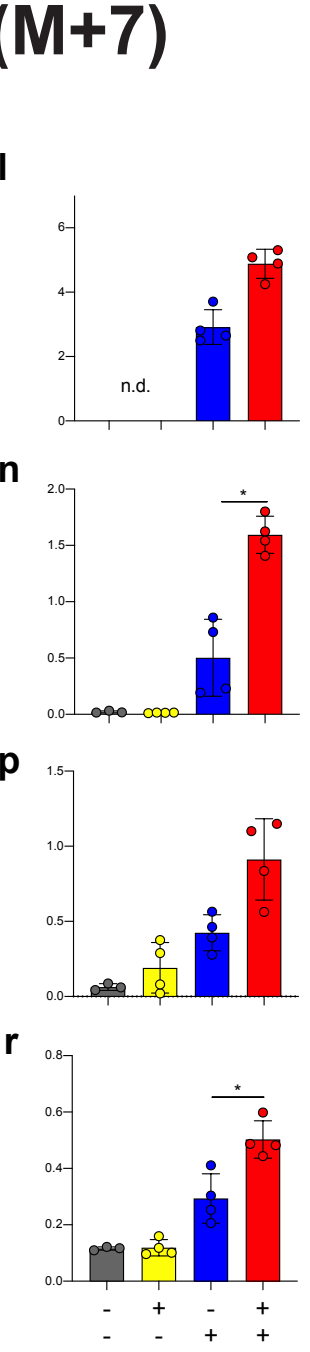
bioRxiv preprint doi: https://doi.org/10.1101/2020.09.10.289561; this version posted September 11,2020. The copyright holder for this preprint (which was not certified by peer review) is the author/funder, who has granted bioRxiv a license to display the preprint in perpetuity. It is made available under aCC-BY-ND 4.0 International license.

a

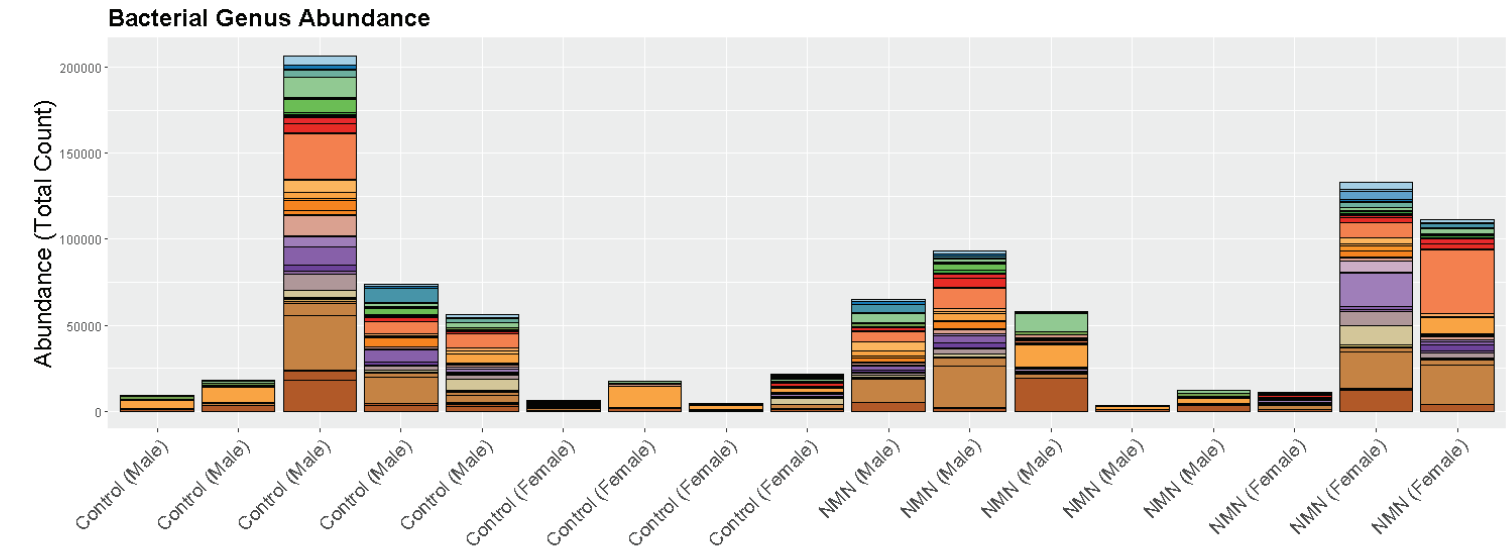

Bacterial Genus Abundance

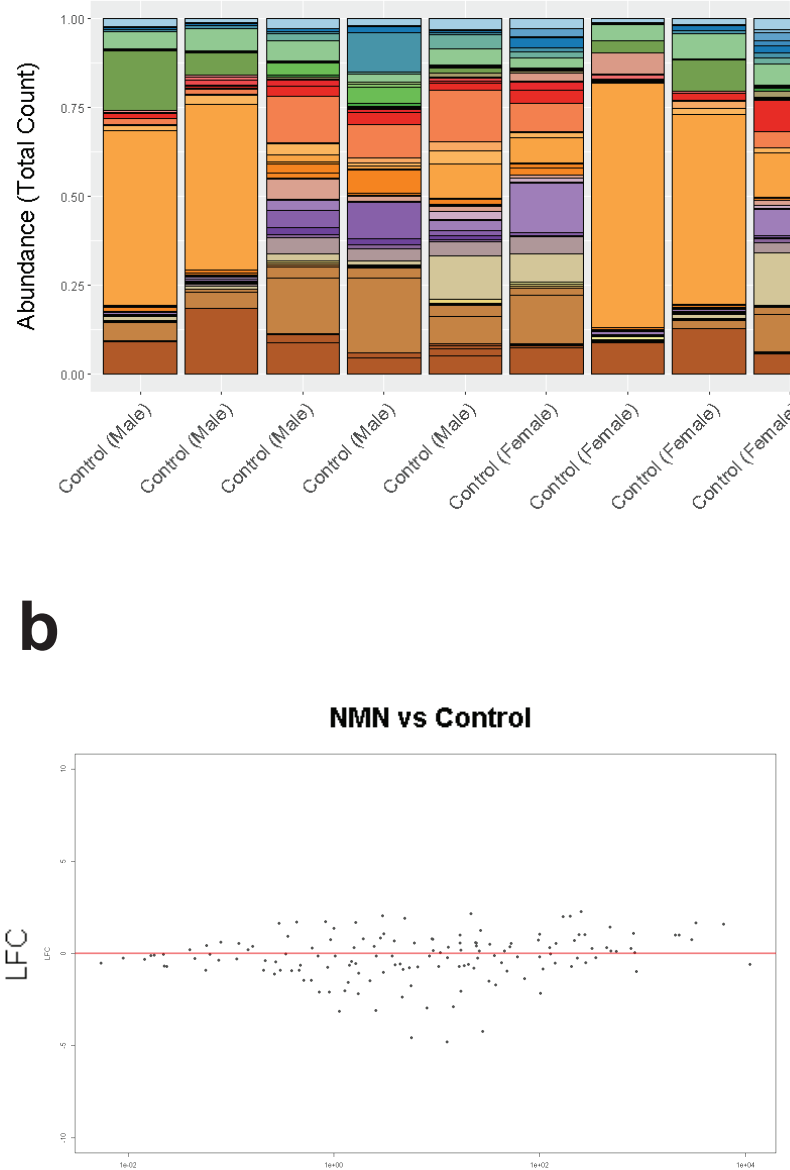

Mean of Normalised Counts
C

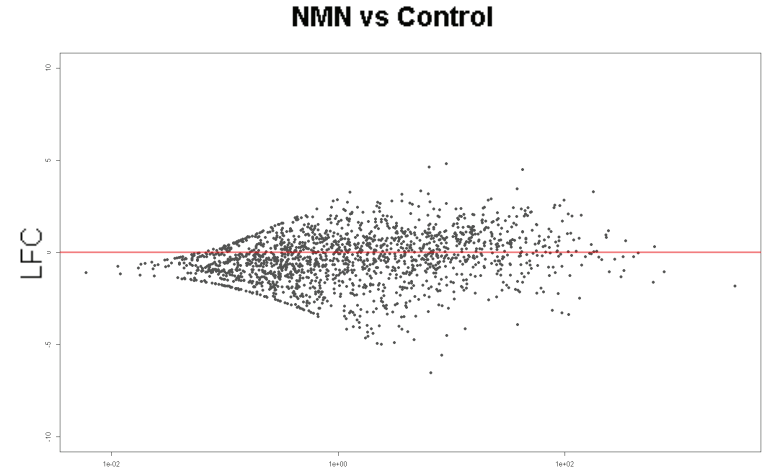

Mean of Normalised Counts

\section{Genus}

$<0.1 \%$ Abund.

Eubacterium] coprostanoligenes group

[Eubacterium] fissicatena group

Eubacterium] ventriosum group

[Eubacterium] xylanophilum group

A2

Alistipes

Ambiguous_taxa

Anaerotruncus

ASF356

Blautia

Butyricicoccus

Butyrivibrio

Candidatus Arthromitus

Christensenellaceae R-7 group

Clostridium sensu stricto 1

Desulfovibrio
Dubosiella

Faecalibaculum

GCA-900066575

Lachnoclostridium

Lachnospiraceae FCSO20 group

Lachnospiraceae NK4A136 group

Lachnospiraceae UCG-001
Lachnospiraceae UCG-006

Lactobacillus

Marvinbryantia

Mucispirillum

Muribaculum

Negativibacillus

Oscillibacter

Papillibacter

Parasutterella

Rikenellaceae RC9 gut group

Roseburia

Ruminiclostridium

Ruminiclostridium

Ruminiclostridium

Ruminococcaceae UCG-014

Turicibacter

UBA1819

uncultured

uncultured

d

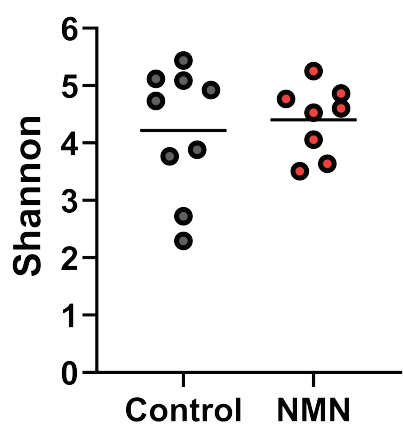


a

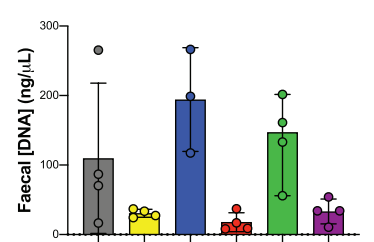

Antibiotics - + - + - +

${ }^{15} \mathrm{~N}-\mathrm{G} \ln (\mathrm{M}+1)-\quad++++$

NMN1 (M+6) - - - - +

C

Bacterial Genus Abundance

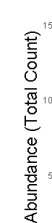

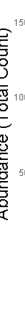

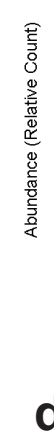

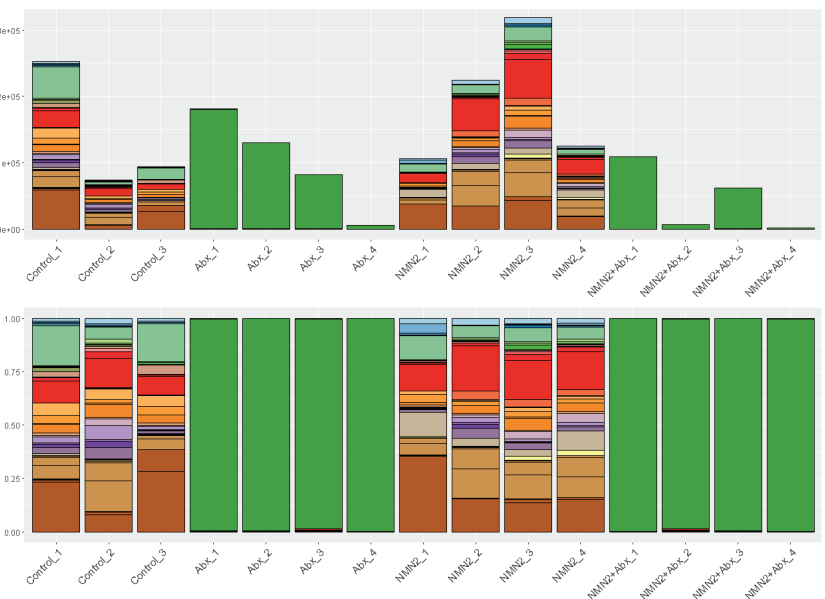

b
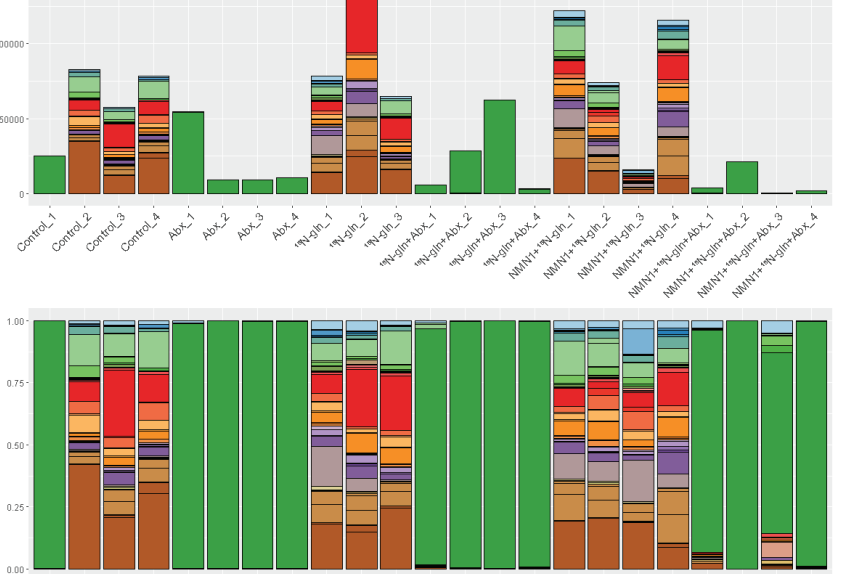

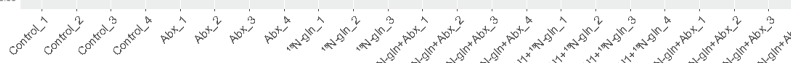

Treatment

d

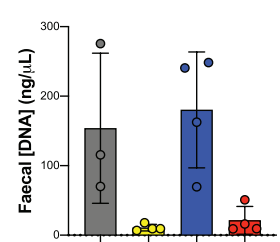

Antibiotics - + - +

NMN2 $(M+7)-\quad++$

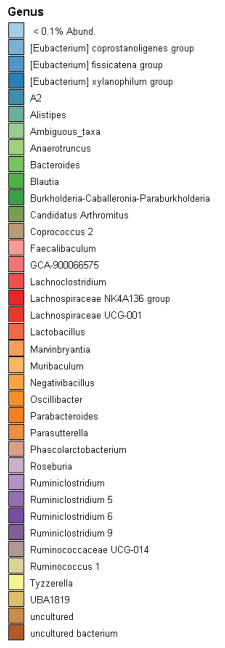


bioRxiv preprint doi: https://doi org/10.1101/2020.09 10.289561; this version posted September 11, 2020. The copyright holder for this preprint (which was not certified by peer review) is the author/funder, who has granted bioRxiv a license to display the preprint in perpetuity. It is made available under aCC-BY-ND 4.0 International license.

a

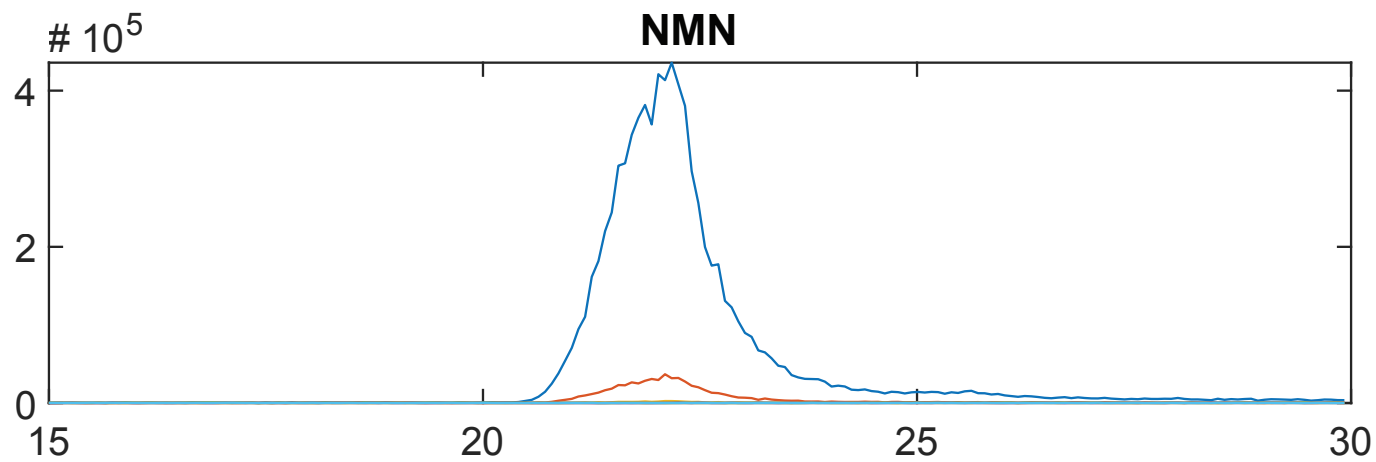

b

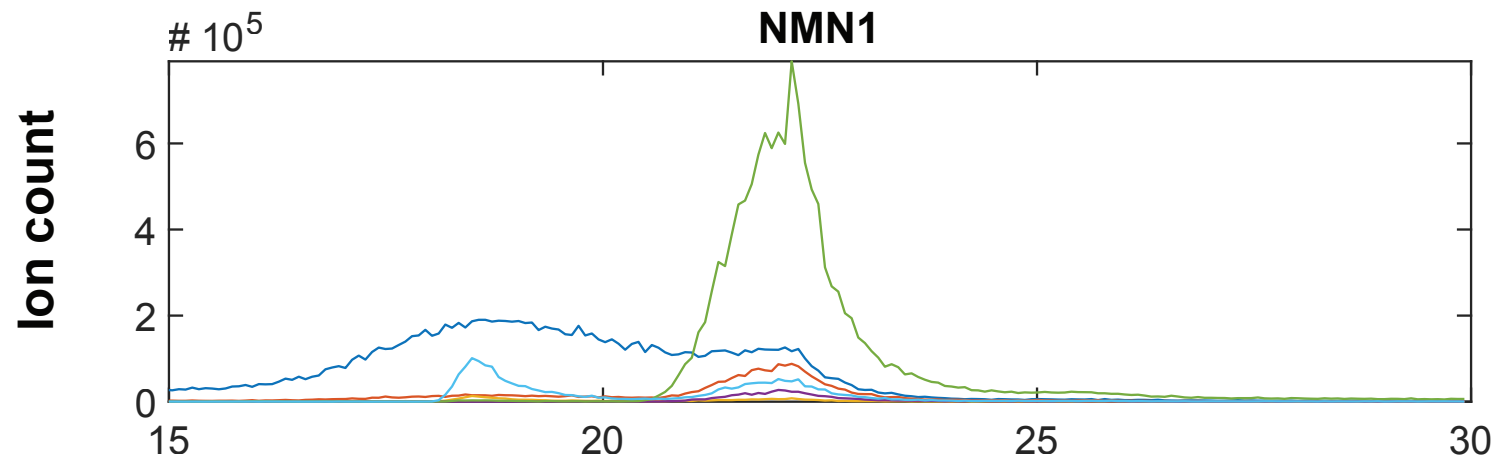

C

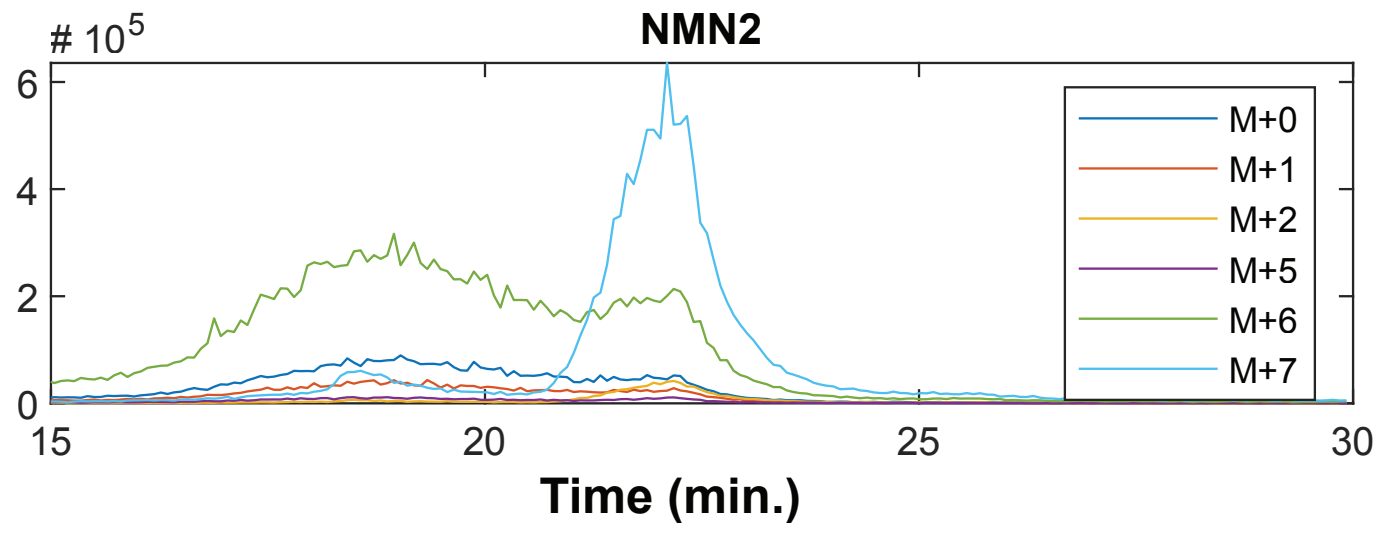

d
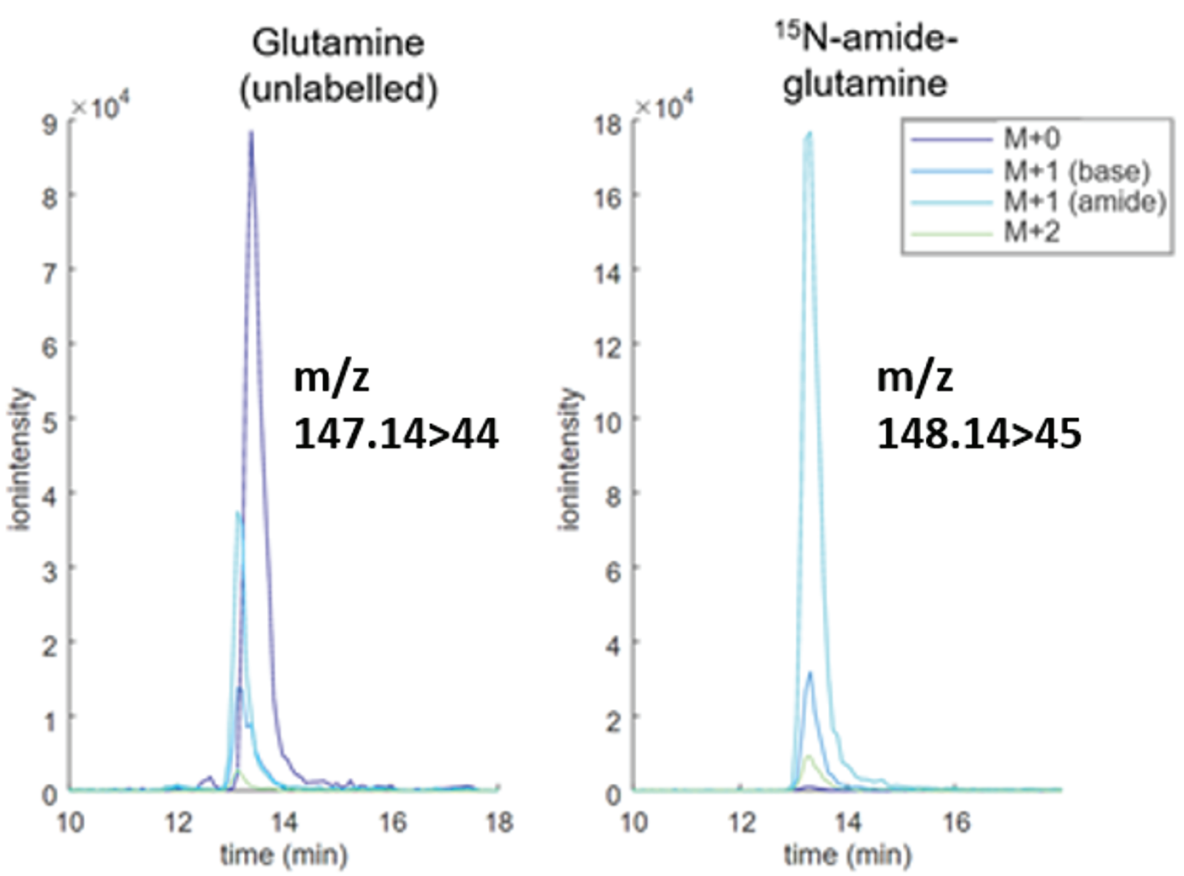
Plasma

a

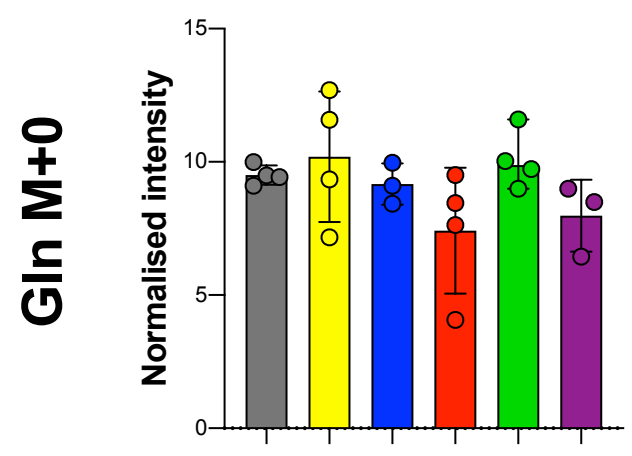

d
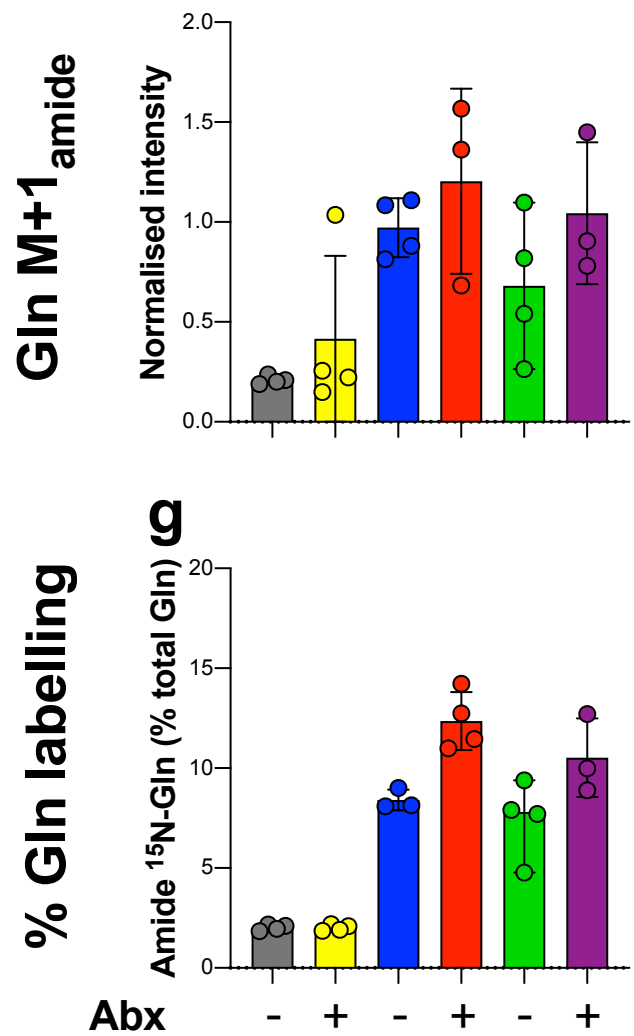

${ }^{15} \mathrm{~N}-\mathrm{G} \ln (\mathrm{M}+1) \quad-\quad-++++$

NMN1 $(M+6)$
Liver

b

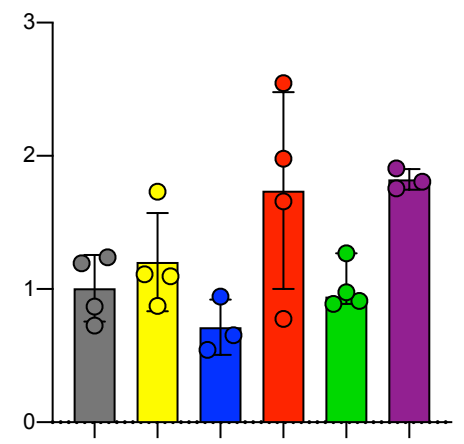

e

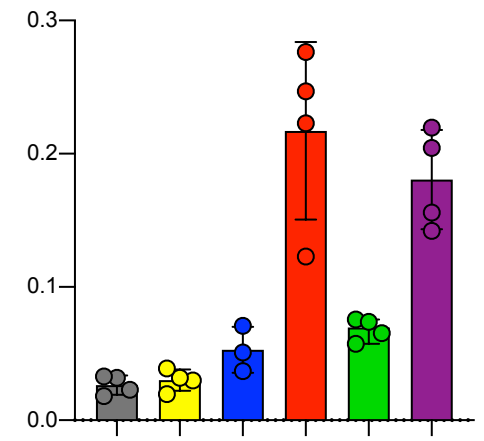

h

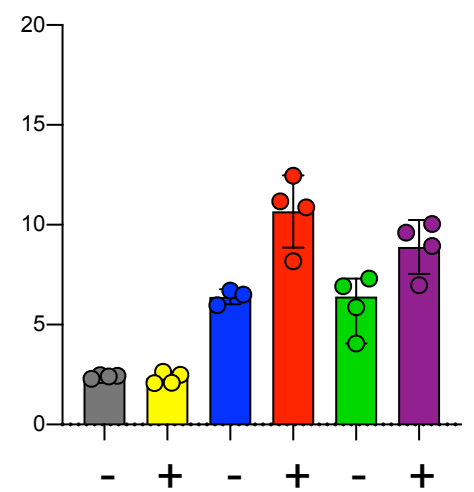

$-\quad++++$

$-\quad-\quad-\quad+\quad+$

\section{C}

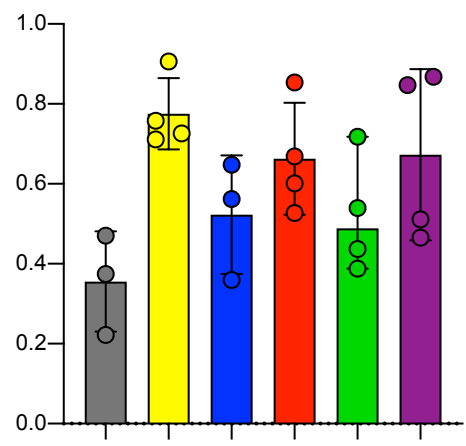

f

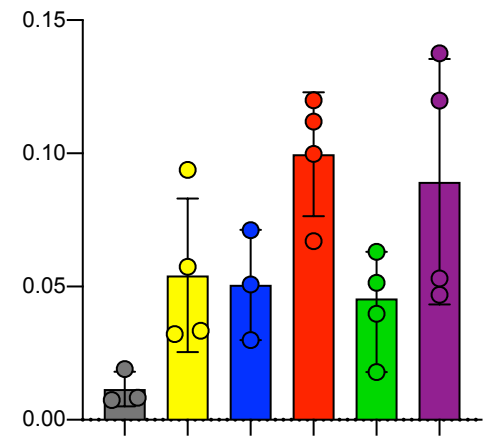

i

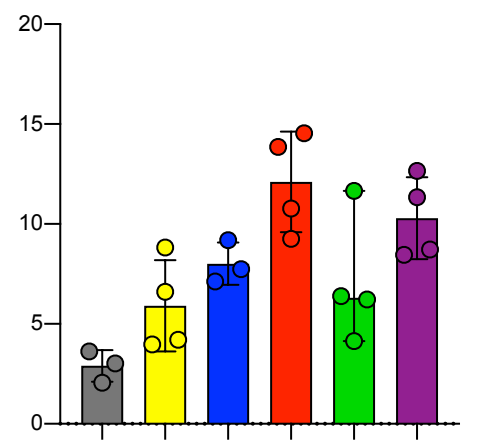

$-+-+\quad+$

$-\quad++++$

GIT 


\section{Endogenous}

a

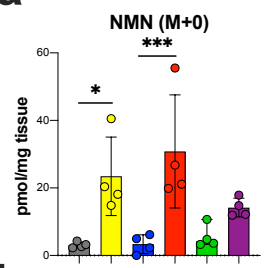

है

b

NAD ${ }^{+}$

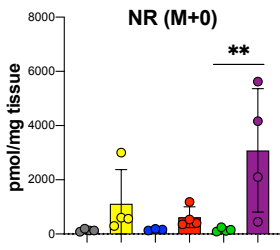

c

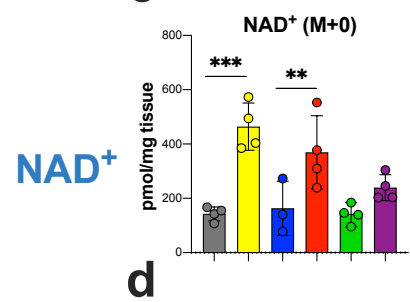

Nam

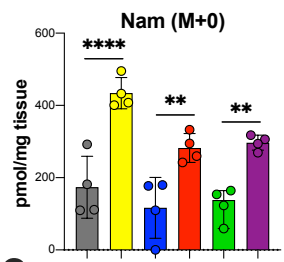

e
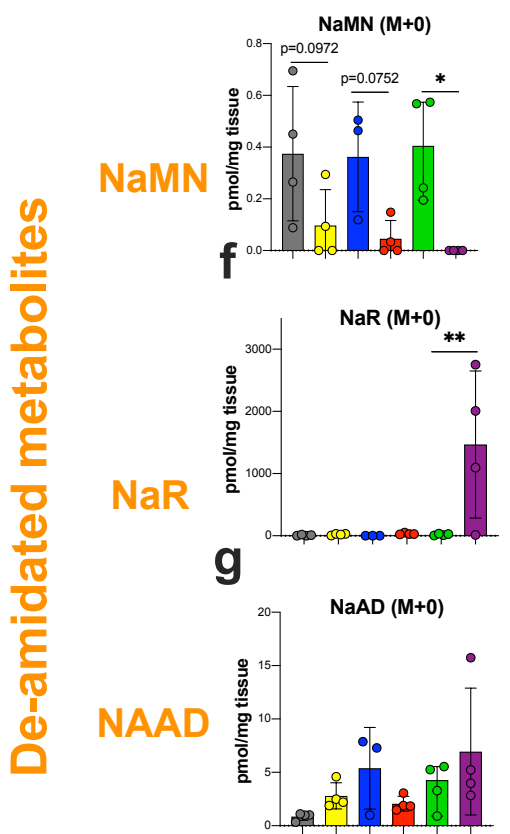

Antibiotics

$-++++$
Intact labelling

h

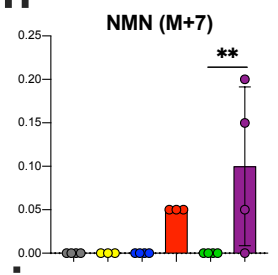

i
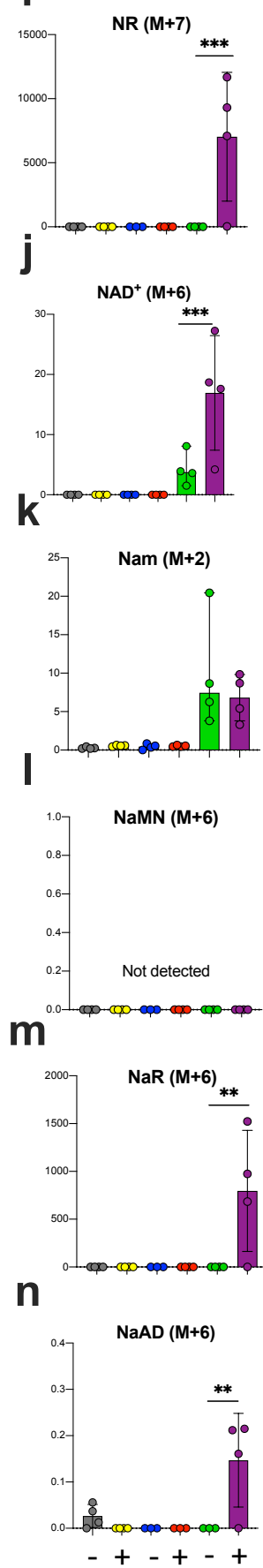

O NMN (M+2)

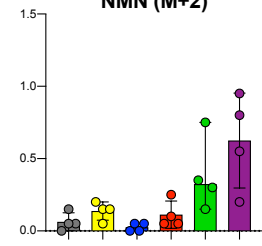

p

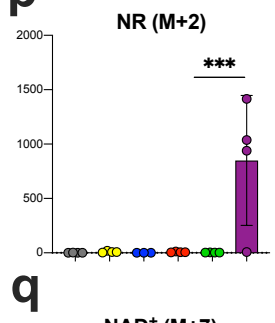

4. $\mathrm{NAD}^{+}(\mathrm{M}+7)$

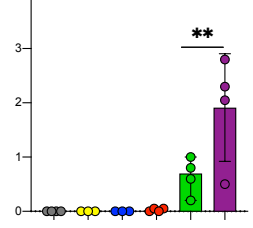

r

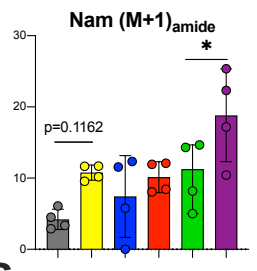

$\mathbf{S}$
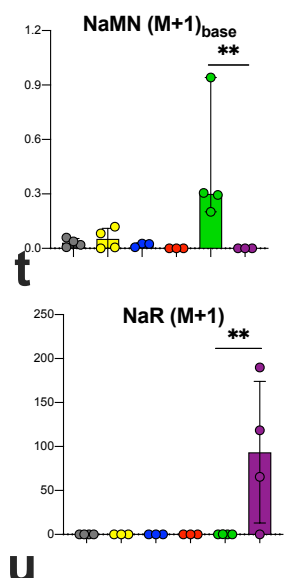

$\mathbf{u}$

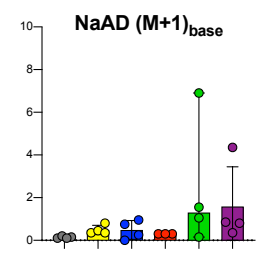

${ }^{15} \mathrm{~N}$-Glutamine $(\mathrm{M}+1) \quad--+++$

NMN1 $(M+6)$ 


\section{Endogenous}

a

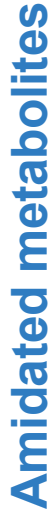

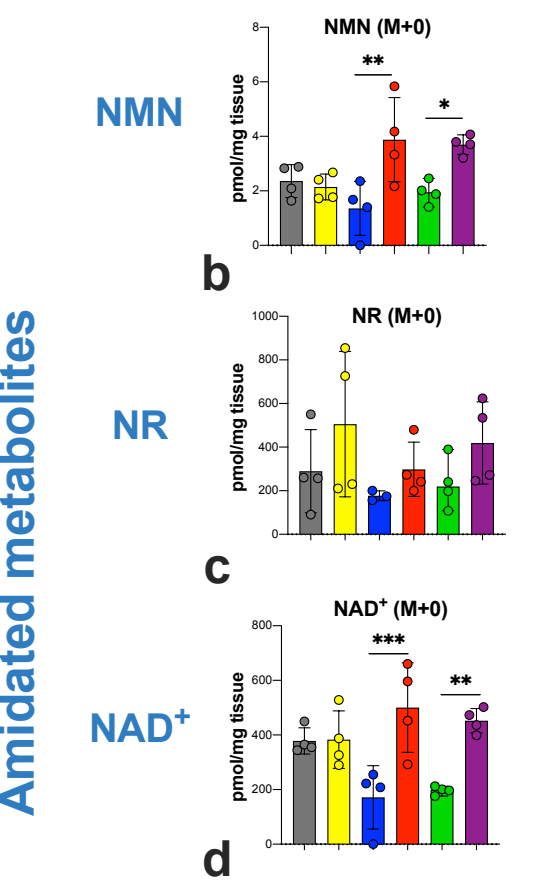

ब्ञ

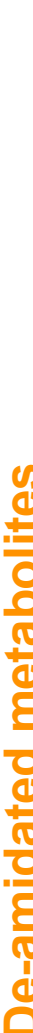

\section{NaMN}

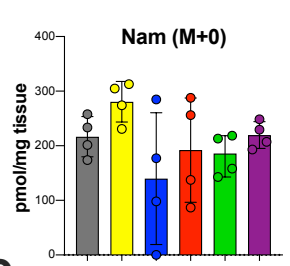

e

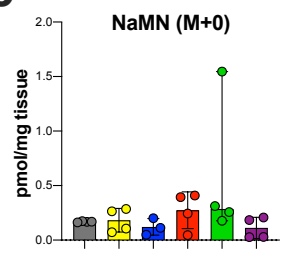

f

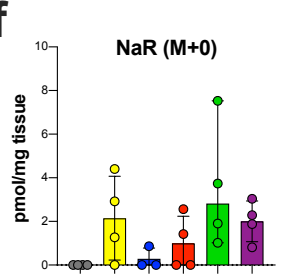

NaR

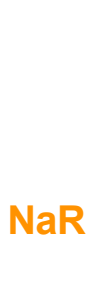

NAAD

9

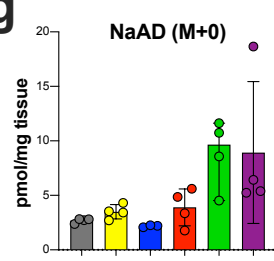

Antibiotics

${ }^{15} \mathrm{~N}$-Glutamine (M+1)

NMN1 $(M+6)$

\section{Intact labelling Partial labelling} h

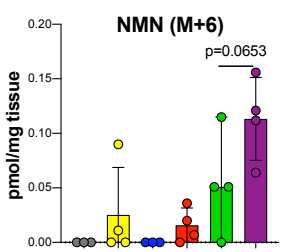

I

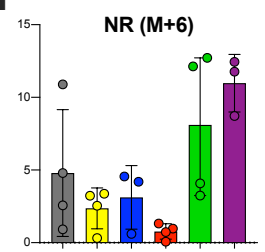

j
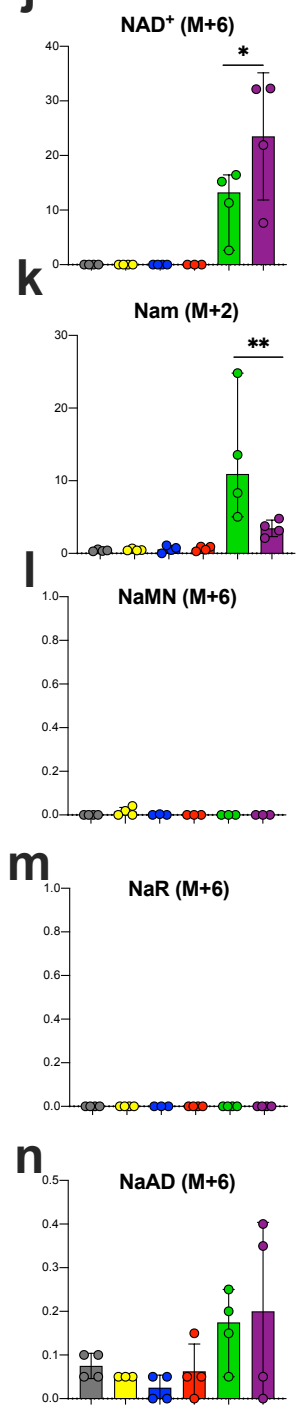

$-+-++$
0

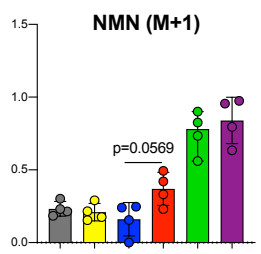

9

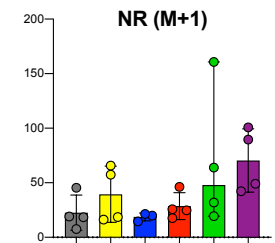

Q
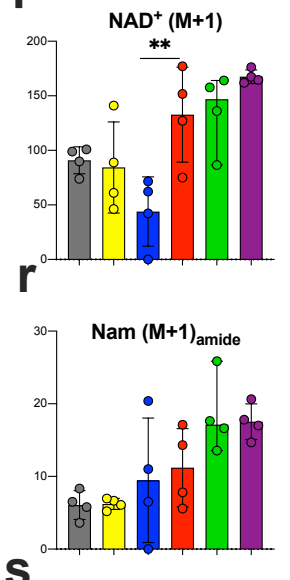

S
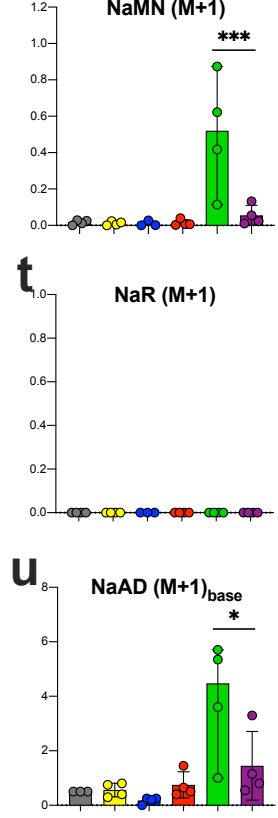


\section{Endogenous}

a

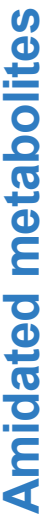

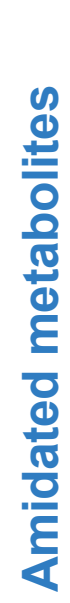

$\mathrm{NAD}^{+}$

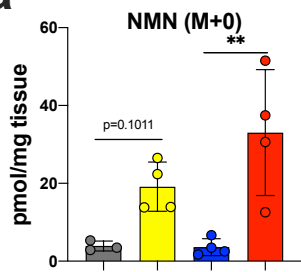

b

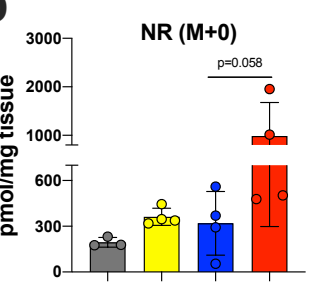

C

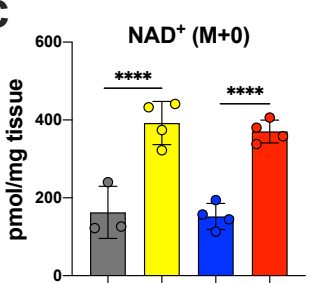

d

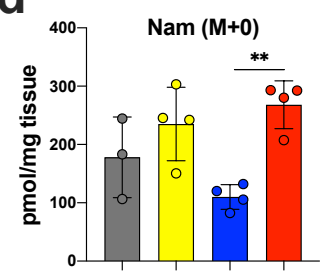

e

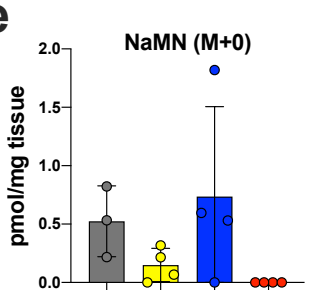

f

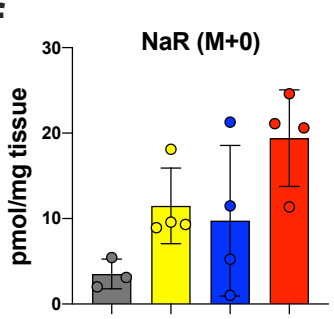

g

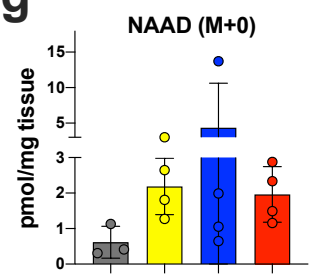

h

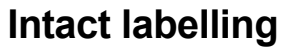

Partial labelling
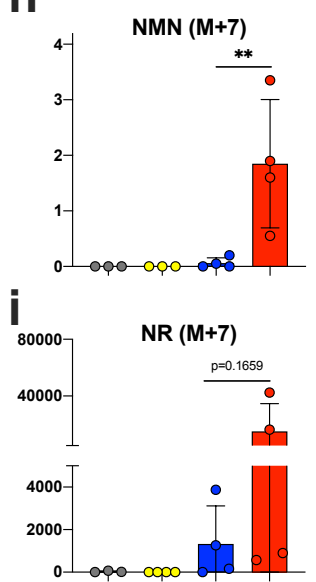

j

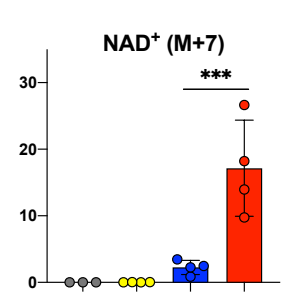

K
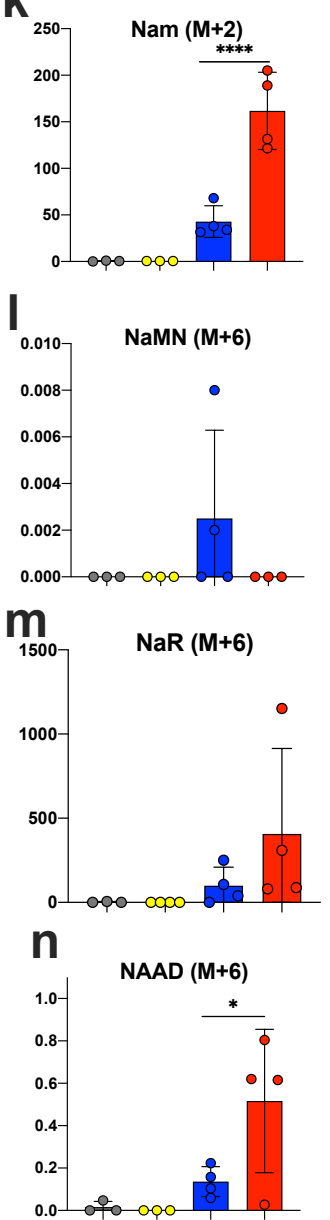

(

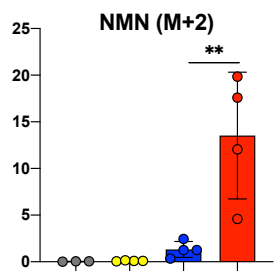

p

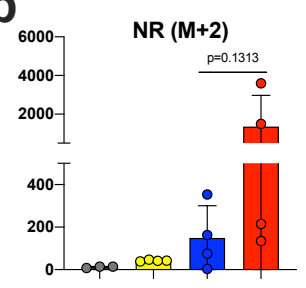

q
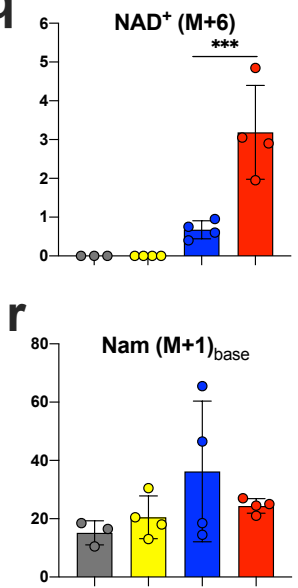

$\mathbf{S}$
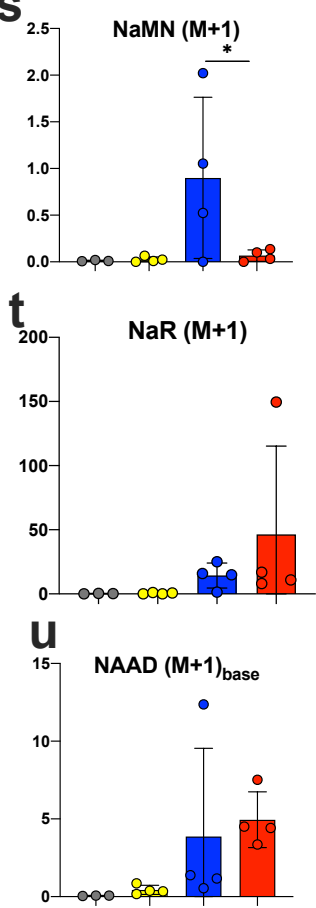
Endogenous

a

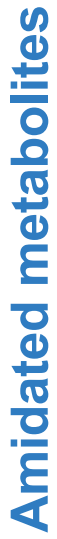
species
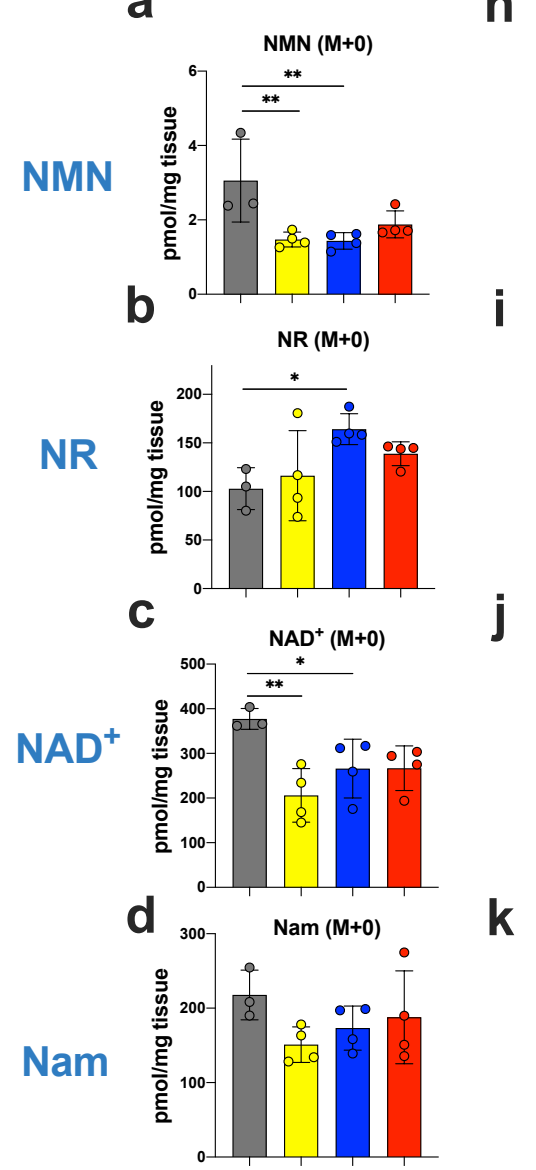

Nam

e
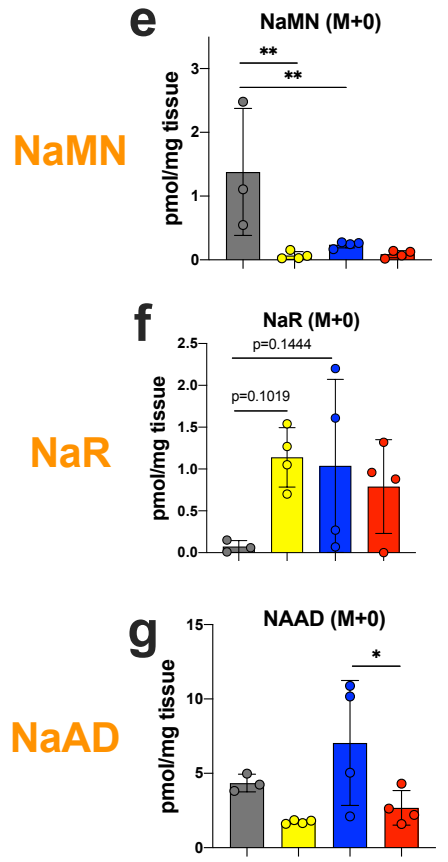

Intact labelled

species

h
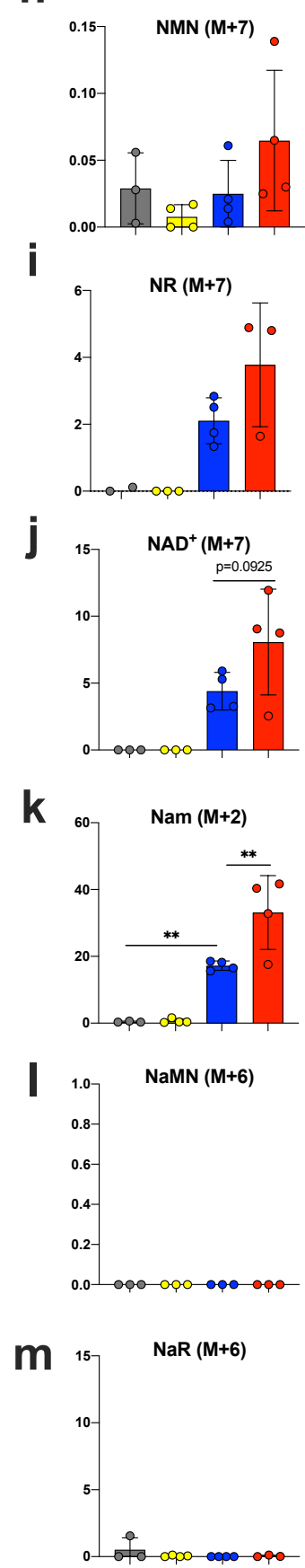

n

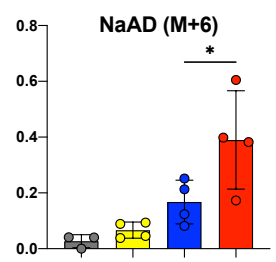

O

Partially labelled species

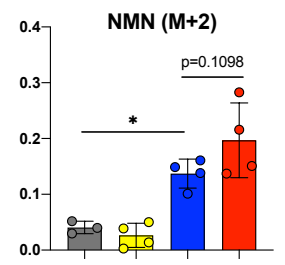

p

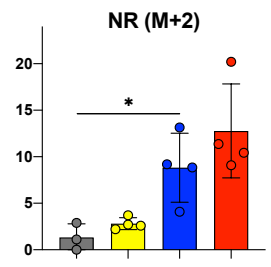

q

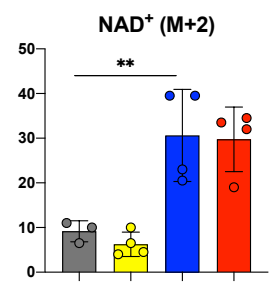

$\Gamma_{120} \mathrm{Nam}(\mathrm{M}+1)_{\text {base }}$
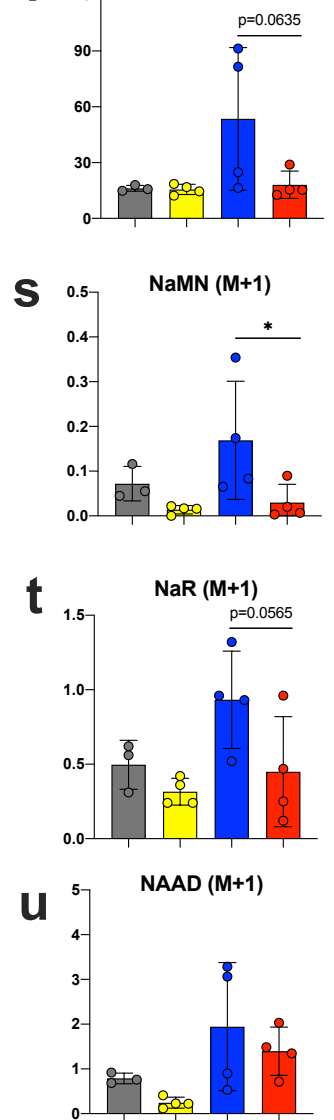
NMN1

$(\mathrm{M}+6)$
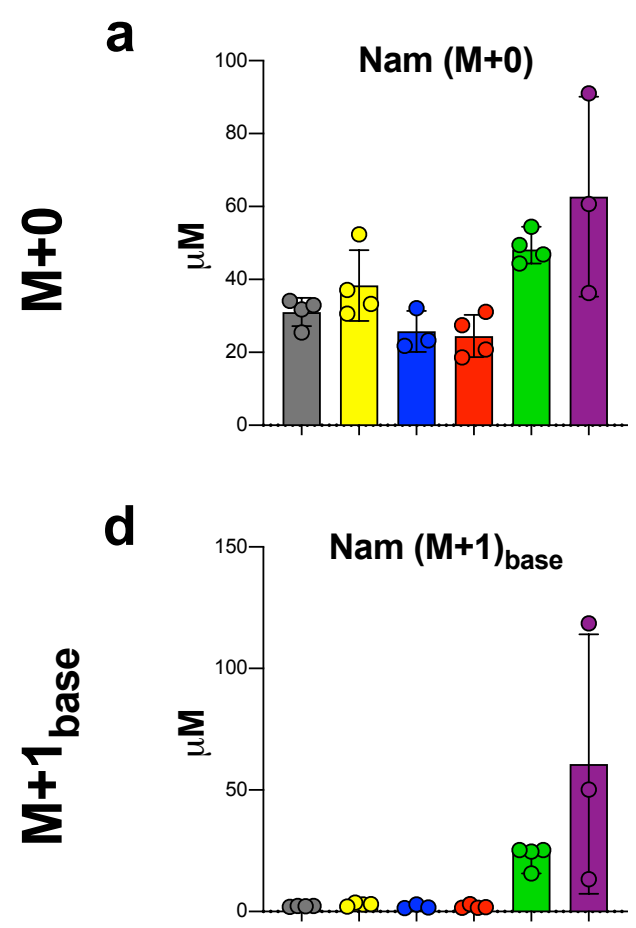

f
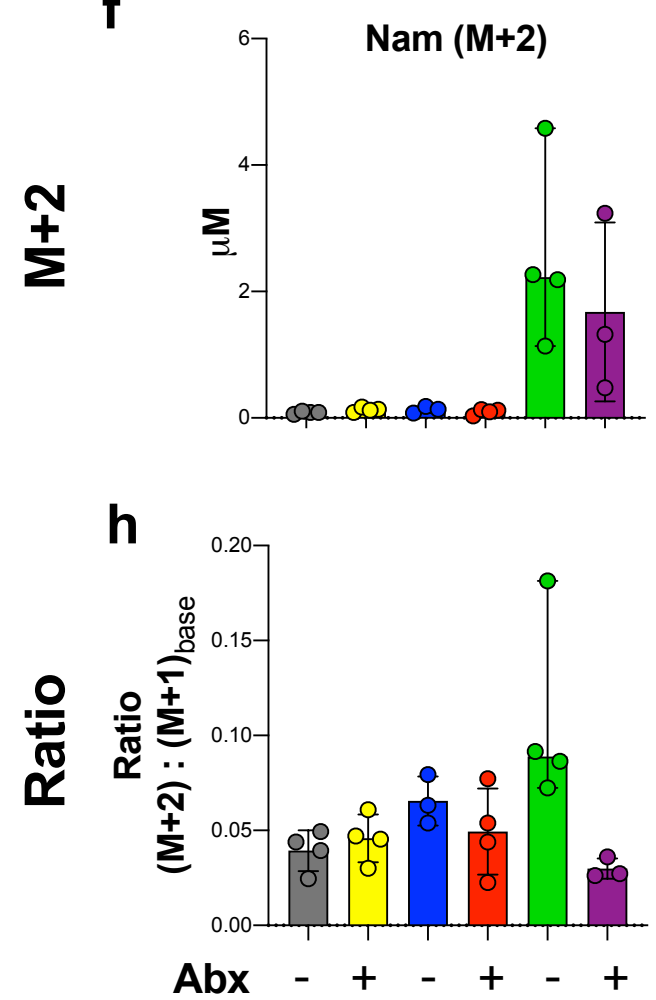

NMN2

$(\mathrm{M}+7)$

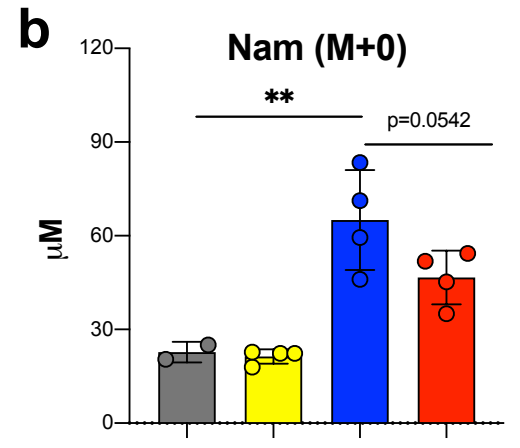

e

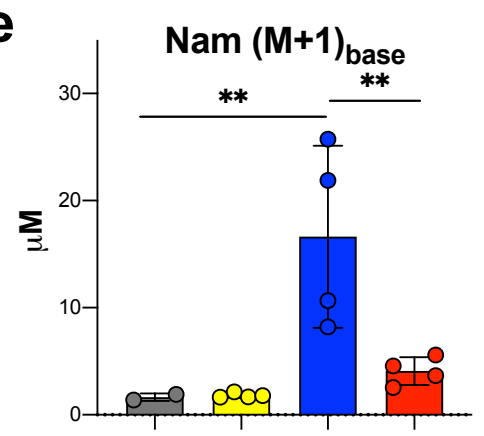

g

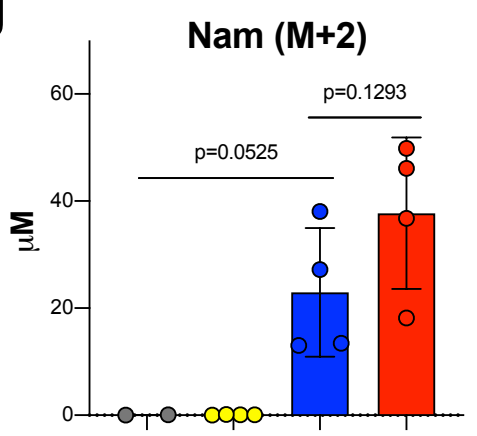

i

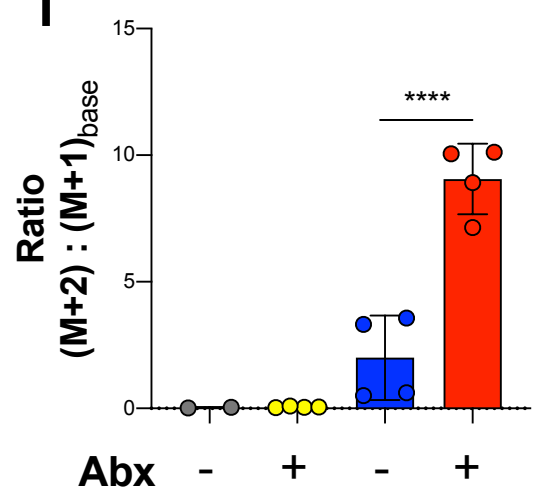

NMN2 - $-\quad+\quad+$

$(\mathrm{M}+7)$
Unlabelled NMN $(\mathrm{M}+0)$

C ${ }^{600} \rightarrow \quad \operatorname{Nam}(\mathbf{M}+\mathbf{0})$

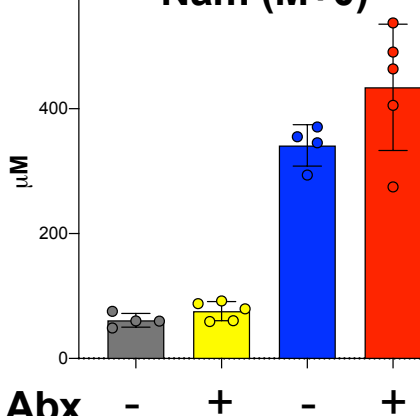

NMN - -++
NMN1 $(M+6) \quad-\quad-\quad-\quad-\quad+\quad+$ 
a

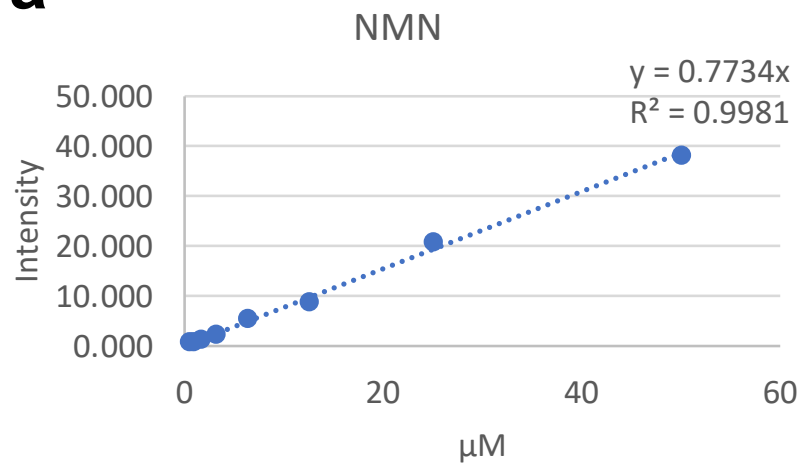

C

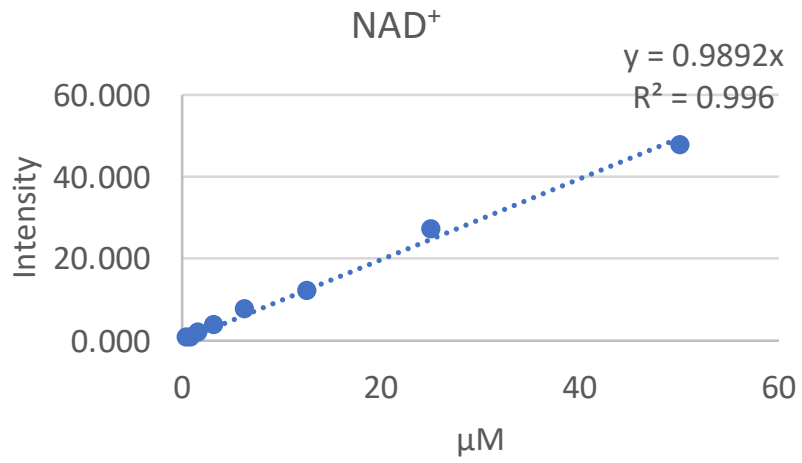

e

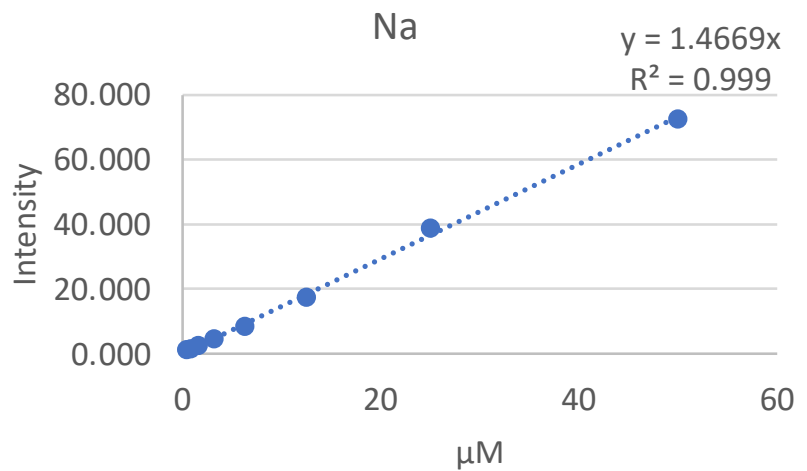

g

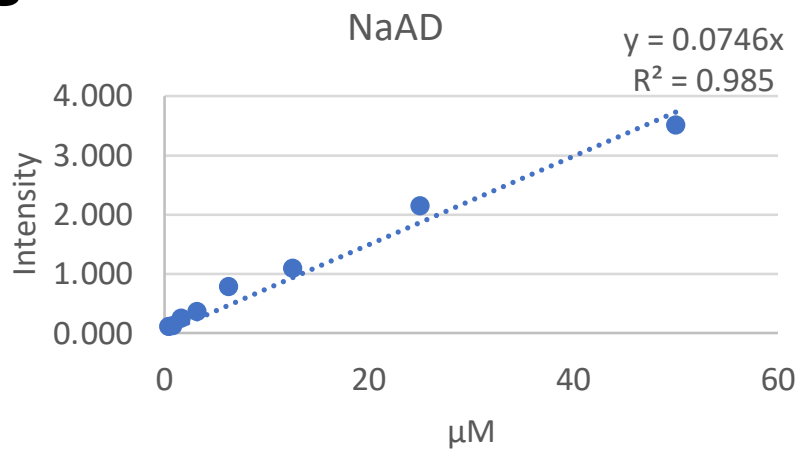

b

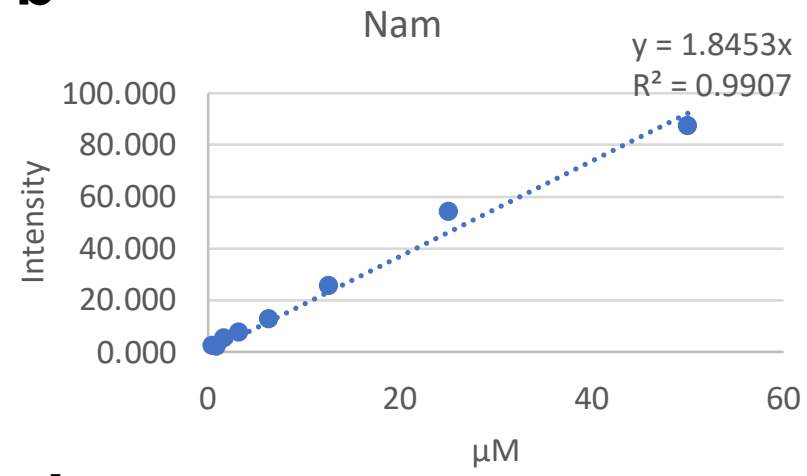

d
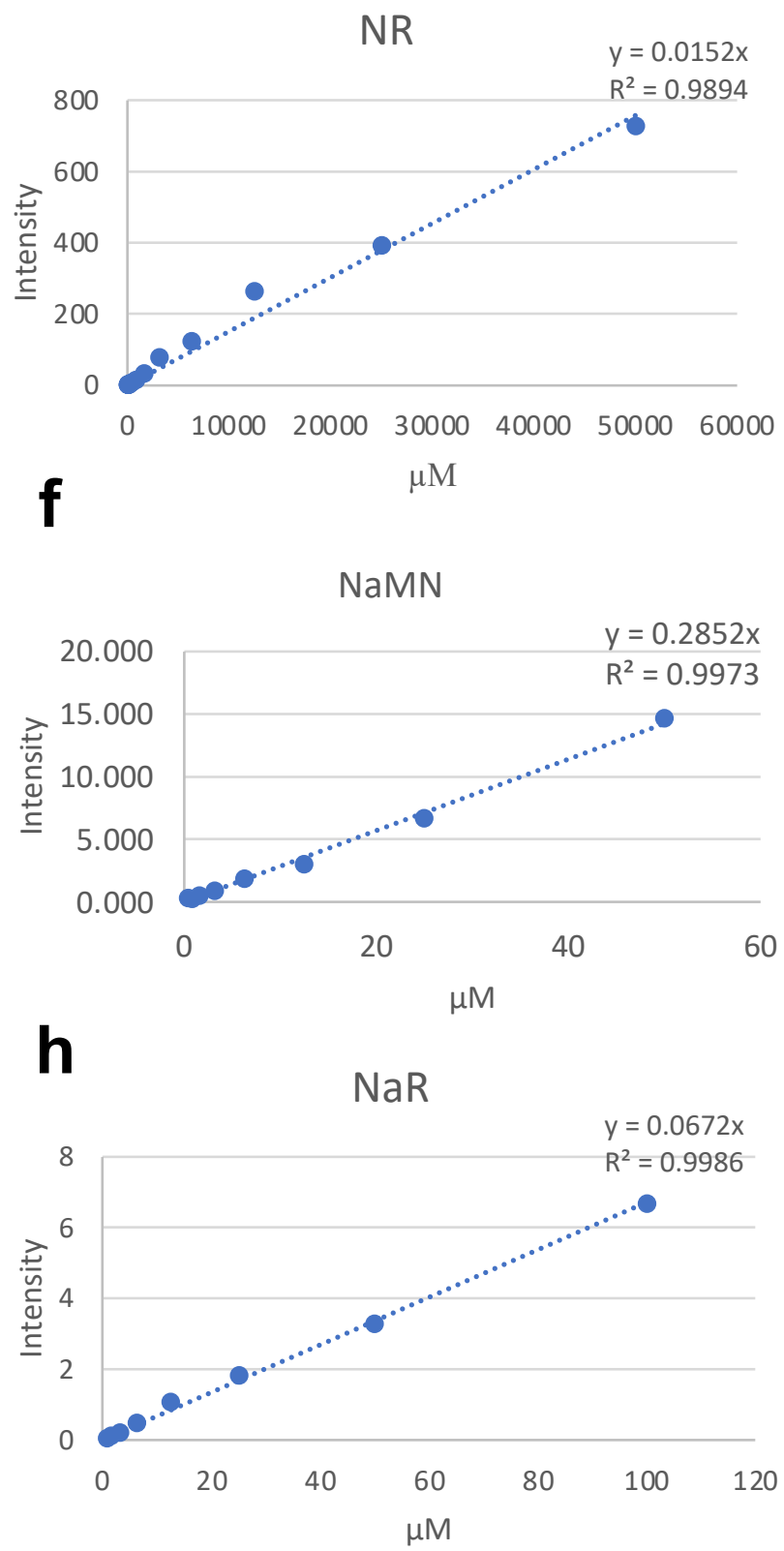

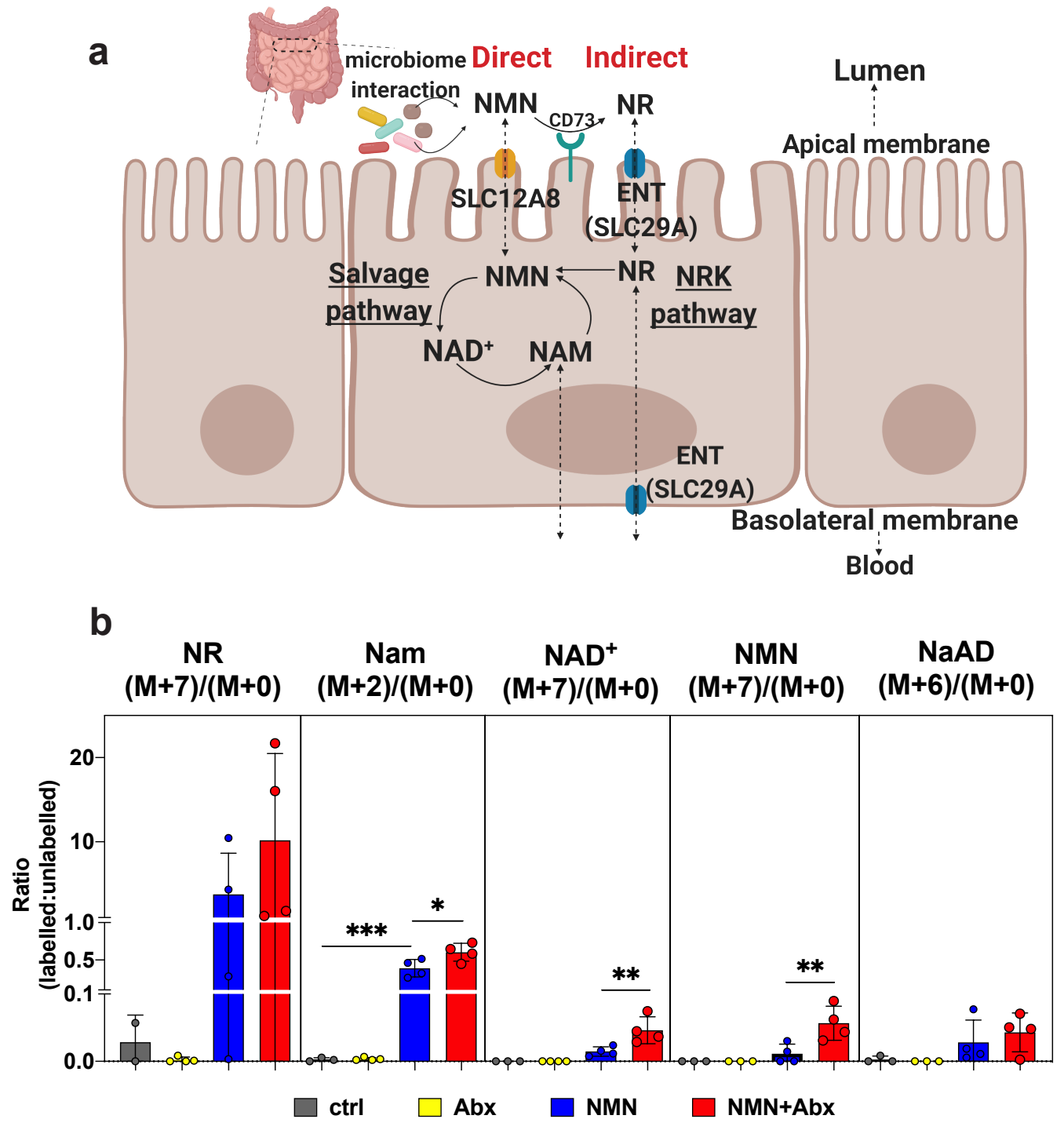
a

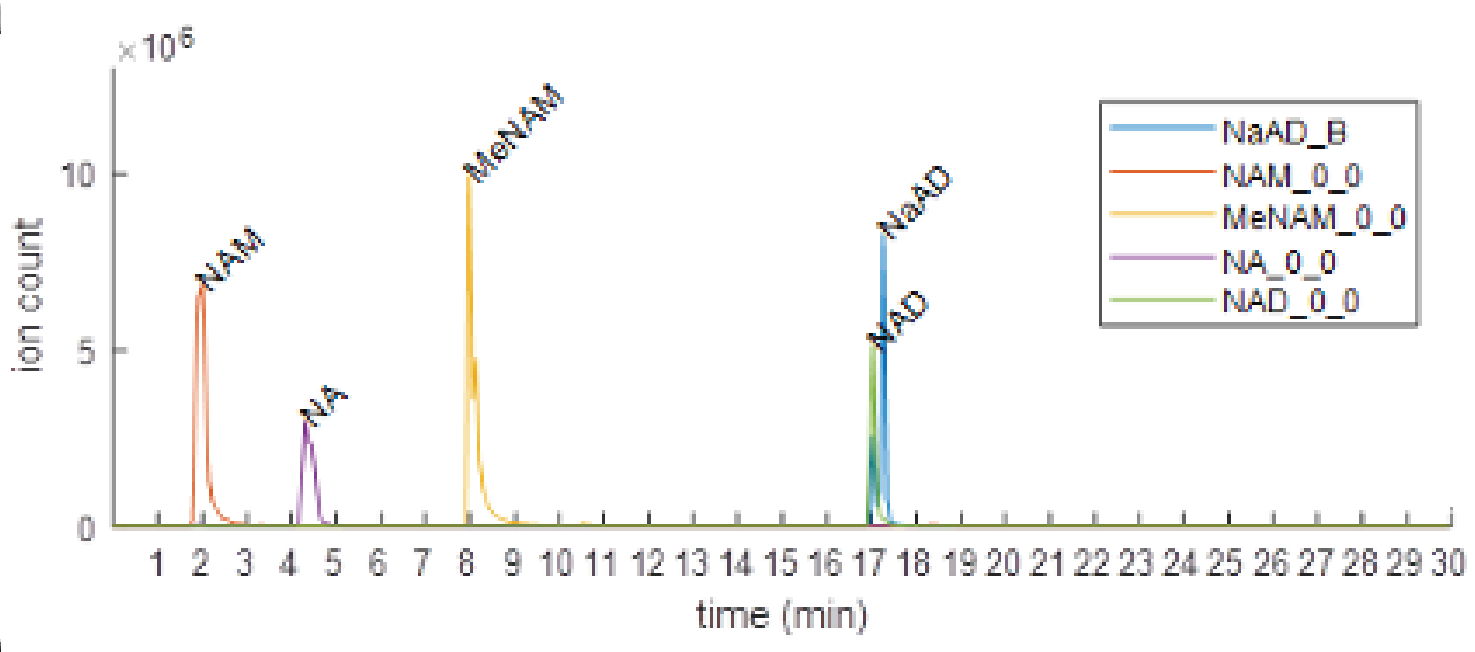

b

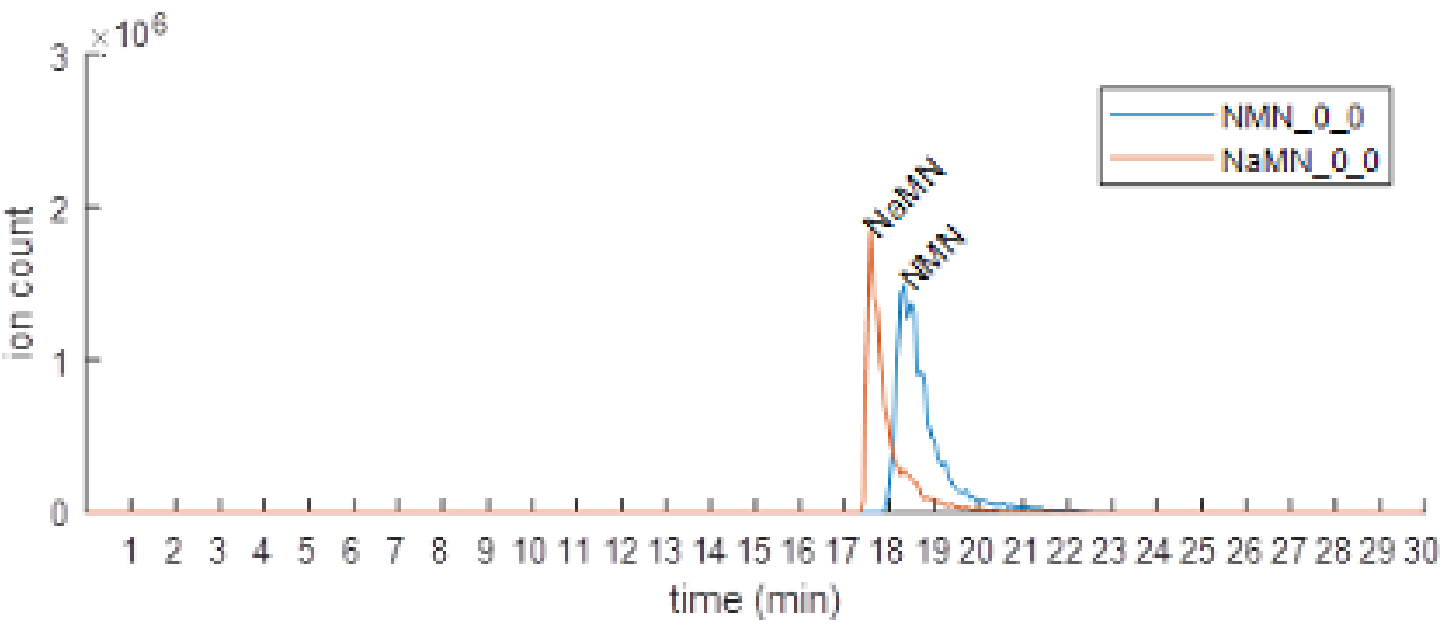

C

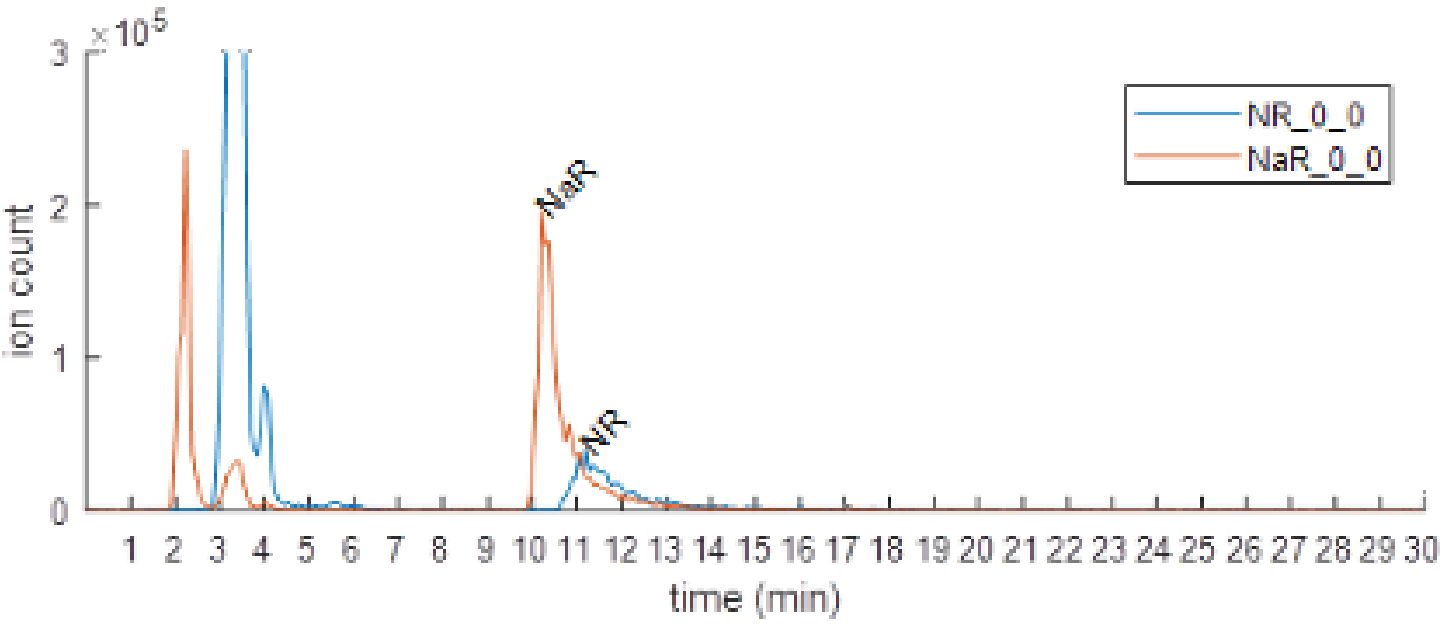

